TRANSACTIONS OF THE

AMERICAN MATHEMATICAL SOCIETY

Volume 356, Number 3, Pages 1045-1119

S 0002-9947(03)03440-8

Article electronically published on September 22, 2003

\title{
SEMILINEAR PARABOLIC EQUATIONS INVOLVING MEASURES AND LOW REGULARITY DATA
}

\author{
H. AMANN AND P. QUITTNER
}

\begin{abstract}
A detailed study of abstract semilinear evolution equations of the form $\dot{u}+A u=\mu(u)$ is undertaken, where $-A$ generates an analytic semigroup and $\mu(u)$ is a Banach space valued measure depending on the solution. Then it is shown that the general theorems apply to a variety of semilinear parabolic boundary value problems involving measures in the interior and on the boundary of the domain. These results extend far beyond the known results in this field. A particularly new feature is the fact that the measures may depend nonlinearly and possibly nonlocally on the solution.
\end{abstract}

\section{INTRODUCTION}

In this paper we extend the theory of linear parabolic evolution problems involving measures, developed in [5], to the case of semilinear evolution equations of the form

$$
\dot{u}+A u=\mu(u) \quad \text { in }[0, T]
$$

where $-A$ generates a strongly continuous analytic semigroup on some Banach space and $\mu(u)$ is a Banach space valued measure depending Lipschitz continuously on $u$. We demonstrate the power of our general results by discussing a number of applications to a variety of nonlinear parabolic boundary value problems involving measures in space and time, which may depend nonlinearly and in a nonlocal way on the solution.

In this introduction we present some simple model problems in order to show the scope of the theory. We restrict ourselves to questions of well-posedness. However, our abstract results, in particular the fact that we prove that the solutions depend continuously on the data, are important for qualitative investigations. This will be illustrated in forthcoming publications, in which applications to control problems are given.

Throughout this paper we assume that $\Omega$ is a nonempty domain in $\mathbb{R}^{n}$ with a compact smooth boundary $\Gamma$, and $\Omega$ lies locally on one side of $\Gamma$ if $\Gamma \neq \emptyset$. Furthermore, $\Gamma_{0}$ and $\Gamma_{1}$ are disjoint and open in $\Gamma$ with $\Gamma_{0} \cup \Gamma_{1}=\Gamma$. Of course, either $\Gamma_{0}$ or $\Gamma_{1}$ can be empty. In this introduction we also suppose that $n \geq 2$.

For a $\sigma$-compact metric space $X$ we denote by $\mathcal{M}(X)$ the Banach space of bounded Radon measures on $X$; that is, $\mathcal{M}(X)=C_{0}(X)^{\prime}$, where $C_{0}(X)$ is the

Received by the editors August 19, 2002.

2000 Mathematics Subject Classification. Primary 35K55, 35K60, 35K90, 28 B05.

Key words and phrases. Nonlinear parabolic systems, weak solutions, measure data, critical exponents. 
space of continuous functions on $X$ vanishing at infinity. If $E$ is a Banach space, then we write $\mathcal{M}_{\text {loc }}\left(\mathbb{R}^{+}, E\right)$ for the space of all $E$-valued Radon measures $\mu$ on $\mathbb{R}^{+}$ such that $\mu \mid[0, T]$ is of bounded variation for each $T>0$. (See [5, Section 2] for precise definitions.)

Given a $\sigma$-compact separable metric space $X$ and a positive Radon measure $\mu$ on $X$, we write $\varphi \in \operatorname{Car}^{1}\left(X \times \mathbb{R}^{m}, \mathbb{R}\right)$ if $\varphi: X \times \mathbb{R}^{m} \rightarrow \mathbb{R}$ is a Carathéodory function such that $\varphi(x, \cdot)$ is continuously differentiable for $\mu$-a.a. $x \in X$, where it is understood from the context which measure $\mu$ we refer to.

Unless stated otherwise, we assume that $f$ belongs to $\operatorname{Car}^{1}\left(\left(\Omega \times \mathbb{R}^{+}\right) \times \mathbb{R}, \mathbb{R}\right)$, satisfies $f(\cdot, \cdot, 0)=0$, and there exist an increasing function $\kappa: \mathbb{R}^{+} \rightarrow \mathbb{R}^{+}$and a constant $\lambda>1$ such that

$$
\left|\partial_{3} f(x, t, \xi)\right| \leq \kappa(t)\left(1+|\xi|^{\lambda-1}\right), \quad(x, t, \xi) \in \Omega \times \mathbb{R}^{+} \times \mathbb{R} .
$$

A prototype of such a function is given by

$$
f(x, t, \xi)=a(x, t)|\xi|^{\lambda-1} \xi, \quad(x, t, \xi) \in \Omega \times \mathbb{R}^{+} \times \mathbb{R},
$$

where $a:=(t \mapsto a(\cdot, t)) \in L_{\infty, \text { loc }}\left(\mathbb{R}^{+}, L_{\infty}(\Omega)\right)$.

We also put

$$
F(u)(x, t):=f(x, t, u(x, t)), \quad u: \Omega \times J \rightarrow \mathbb{R}, \quad(x, t) \in \Omega \times J,
$$

where, in general, $J$ is a nontrivial subinterval of $\mathbb{R}^{+}$containing 0 .

In this introduction we consider the parabolic problem

$$
\begin{aligned}
\partial_{t} u-\Delta u & =F(u)+\mu_{\Omega}(u) & & \text { in } & \Omega \times \mathbb{R}^{+}, \\
u & =\mu_{0}(u) & & \text { on } & \Gamma_{0} \times \mathbb{R}^{+}, \\
\partial_{\nu} u & =\mu_{1}(u) & & \text { on } & \Gamma_{1} \times \mathbb{R}^{+},
\end{aligned}
$$

with $\partial_{\nu}$ being the derivative with respect to the outer unit normal on $\Gamma$. (Of course, here and everywhere else in this paper, all explicit or implicit references to $\Gamma$ have to be neglected if $\Omega=\mathbb{R}^{n}$.) Furthermore, given a suitably regular function $v$ on $\mathbb{R}^{+}$,

$$
\vec{\mu}(v):=\left(\mu_{\Omega}(v), \mu_{0}(v), \mu_{1}(v)\right) \in \mathcal{M}_{\mathrm{loc}}\left(\mathbb{R}^{+}, \mathcal{M}(\Omega) \times \mathcal{M}\left(\Gamma_{0}\right) \times \mathcal{M}\left(\Gamma_{1}\right)\right),
$$

where the map $v \mapsto \vec{\mu}(v)$ is always supposed to possess the Volterra property, meaning that, given any $T>0$, up to time $T$ the measure $\mu(v)$ depends on the values of $v$ up to time $T$ only, that is, $\vec{\mu}(v) \mid[0, T]$ depends on $v \mid[0, T]$ only. In other words, (0.3) is a semilinear heat equation involving measures (in space and time) in the domain $\Omega$ and on the boundary $\Gamma$, which may depend nonlinearly and nonlocally on the solution.

We suppose that $1<q<\infty$ and $1 \leq r<\infty$. By a (weak) $L_{r}\left(L_{q}\right)$-solution of (0.3) on $J$ we mean a $u \in L_{r, \text { loc }}\left(J, L_{q}(\Omega)\right)$ such that

$$
\begin{aligned}
\int_{\Omega \times J}\left(-\partial_{t} \varphi-\Delta \varphi\right) u d x d t= & \int_{\Omega \times J} \varphi F(u) d x d t+\int_{\Omega \times J} \varphi d \mu_{\Omega}(u) \\
& -\int_{\Gamma_{0} \times J} \partial_{\nu} \varphi d \mu_{0}(u)+\int_{\Gamma_{1} \times J} \varphi d \mu_{1}(u)
\end{aligned}
$$

for every $\varphi \in \mathcal{D}\left(\bar{\Omega} \times J^{*}\right)$ satisfying

$$
\varphi(\cdot, t)\left|\Gamma_{0}=0, \quad \partial_{\nu} \varphi(\cdot, t)\right| \Gamma_{1}=0, \quad t \in J,
$$


where $J^{*}:=J \backslash\{\sup J\}$, and $\mathcal{D}$ is the space of smooth functions having compact support in the indicated domain. Clearly, $u$ is a $C\left(L_{q}\right)$-solution if $u \in C\left(J, L_{q}(\Omega)\right)$, and $u$ satisfies (0.5).

Besides (0.4), we also consider the more regular case where

$$
\vec{\mu}(v) \in L_{r, \text { loc }}\left(\mathbb{R}^{+}, \mathcal{M}(\Omega) \times \mathcal{M}\left(\Gamma_{0}\right) \times \mathcal{M}\left(\Gamma_{1}\right)\right) .
$$

In this case we study the initial boundary value problem

$$
\begin{aligned}
& \partial_{t} u-\Delta u=F(u)+\mu_{\Omega}(u) \quad \text { in } \quad \Omega \times(0, \infty) \text {, } \\
& u=\mu_{0}(u) \quad \text { on } \quad \Gamma_{0} \times(0, \infty), \\
& \partial_{\nu} u=\mu_{1}(u) \quad \text { on } \quad \Gamma_{1} \times(0, \infty), \\
& u(\cdot, 0)=u^{0} \quad \text { on } \quad \Omega .
\end{aligned}
$$

Note that (0.7) is a special case of (0.3), of course, since we can replace $\mu_{\Omega}(u)$ in (0.3) by $\mu_{\Omega}(u)+u^{0} \otimes \delta_{0}$, with $\delta_{0}$ being the Dirac measure with support in $t=0$. Thus it is clear what is meant by an $L_{r}\left(L_{q}\right)$ - or a $C\left(L_{q}\right)$-solution of (0.7).

In the following theorem we collect - somewhat informally — some of the main results proven in this paper for problems (0.3) and (0.7). Precise formulations of the various continuity properties are given in Theorems 1.2 and 2.2. Of course, $\xi^{\prime}:=\xi /(\xi-1)$ is the dual exponent of $\xi \in[1, \infty]$, and $B U C$ is the space of bounded and uniformly continuous functions. Moreover, Lipschitz continuity is always understood in the local sense.

Theorem 0.1. $\quad$ (i) Suppose that

$$
\lambda<(n+2) /(n+1)
$$

and that the map $v \mapsto \vec{\mu}(v)$ is Lipschitz continuous from $L_{\lambda, \text { loc }}\left(\mathbb{R}^{+}, L_{\lambda}\right)$ into $\mathcal{M}_{\text {loc }}\left(\mathbb{R}^{+}, \mathcal{M}(\Omega) \times \mathcal{M}\left(\Gamma_{0}\right) \times \mathcal{M}\left(\Gamma_{1}\right)\right)$. Then (0.3) has a unique maximal $L_{\lambda}\left(L_{\lambda}\right)$-solution $\bar{u}$. It depends Lipschitz continuously on $\vec{\mu}$ and $f$, and it is positive if $\vec{\mu}$ is positive.

(ii) Assume that

$$
\lambda<n /(n-1), \quad r>2 /\left(1-n / \lambda^{\prime}\right),
$$

and that $v \mapsto \vec{\mu}(v)$ is locally Lipschitz continuous from $C\left(\mathbb{R}^{+}, L_{\lambda}(\Omega)\right)$ into $L_{r, \text { loc }}\left(\mathbb{R}^{+}, \mathcal{M}(\Omega) \times \mathcal{M}\left(\Gamma_{0}\right) \times \mathcal{M}\left(\Gamma_{1}\right)\right)$. Then problem (0.7) has for each $u^{0} \in W_{\lambda}^{1-n / \lambda^{\prime}}$ a unique maximal $C\left(L_{\lambda}\right)$-solution $\bar{u}$. It depends Lipschitz continuously on $u^{0}, \vec{\mu}$, and $f$, and it is positive if $u^{0}$ and $\vec{\mu}$ are positive.

(iii) In each of the above cases, $J(\bar{u})$, the interval of existence of $\bar{u}$, is open in $\mathbb{R}^{+}$.

(iv) If the hypotheses of (i) are satisfied, suppose that

$$
\bar{u} \in L_{\lambda}\left((0, T) \cap J(\bar{u}), L_{\lambda}(\Omega)\right) \text { for every } T>0 ;
$$

otherwise assume that

$$
\bar{u} \in B U C\left([0, T] \cap J(\bar{u}), L_{\lambda}(\Omega)\right) \text { for every } T>0 .
$$

Then $J(\bar{u})=\mathbb{R}^{+}$, that is, $\bar{u}$ is a global solution.

Remark 0.2. This theorem is a particular case of the much more general Theorem [5.1] (also see Proposition 5.9] and, for the continuity assertion, Theorems 3.2 [3.4). In fact, it allows for the following generalizations. 
(a) The assumption that $\mu_{\Omega}(v)$ and $\mu_{1}(v)$ are measures in $x$ and $t$ can be replaced by the hypotheses that $\mu_{\Omega}(v)$ and $\mu_{1}(v)$ are measures on $\mathbb{R}^{+}$with values in $\mathcal{M}^{-1}(\Omega)$ and $\mathcal{M}^{-1}\left(\Gamma_{1}\right)$, respectively, where $\mathcal{M}^{-1}(\Omega)$ is the dual of the space of all $C^{1}$-functions $v$ on $\bar{\Omega}$ vanishing at infinity and satisfying $v \mid \Gamma_{0}=0$ and $\partial_{\nu} v \mid \Gamma_{1}=0$, and $\mathcal{M}^{-1}\left(\Gamma_{1}\right):=C^{1}\left(\Gamma_{1}\right)^{\prime}$.

(b) In the intermediate case, where

$$
\vec{\mu}(v) \in L_{r, \text { loc }}\left(\mathbb{R}^{+}, \mathcal{M}(\Omega) \times \mathcal{M}\left(\Gamma_{0}\right) \times \mathcal{M}\left(\Gamma_{1}\right)\right)
$$

with $1 \leq r<2 /\left(1-n / \lambda^{\prime}\right)$, one gets an upper bound for $\lambda$ lying between the values $(n+2) /(n+1)$ and $n /(n-1)$ and depending on $r$, as well (see Theorem5.1). Furthermore, the uniform boundedness assumption for $f$ with respect to the variable $x \in \Omega$ can be replaced by an integrability condition.

(c) If $\mu_{0}=0$, that is, in the case of homogeneous Dirichlet data, we can replace the condition on $\lambda$ in (0.8) by $\lambda<1+2 / n$ and (0.9) by $\lambda<1+2 /(n-2)$, respectively. Furthermore, $f$ may depend nonlinearly on the gradient of $u$ as well, and $\mu_{1}(v)$ can be replaced by $\mu_{1}(v)+G(v)$, where $g \in \operatorname{Car}^{1}\left(\left(\Gamma_{1} \times \mathbb{R}^{+}\right) \times \mathbb{R}, \mathbb{R}\right)$, satisfying a suitable growth restriction similar to the one given in (0.2), and where $G(v)(x, t):=g(x, t, v(x, t))$. In this case the unique maximal solution satisfies $u(\cdot, t) \in W_{q}^{1}(\Omega)$ for almost all $t$ in the interval of existence and a properly chosen $q$. Thus it is a weak solution of (0.3) in the sense that

$$
\begin{aligned}
\int_{\Omega \times J} & \left\{-\left(\partial_{t} \varphi\right) v+\nabla \varphi \cdot \nabla v\right\} d x d t \\
= & \int_{\Omega \times J} \varphi F(u) d x d t+\int_{\Omega \times J} \varphi d \mu_{\Omega}(u)+\int_{\Gamma_{1} \times J} \varphi G(u) d \sigma d t+\int_{\Gamma_{1} \times J} \varphi d \mu_{1}(u)
\end{aligned}
$$

for all $\varphi \in \mathcal{D}\left(\bar{\Omega} \times J^{*}\right)$ vanishing on $\Gamma_{0} \times J$, where $d \sigma$ is the volume measure of $\Gamma$ (cf. Theorem 6.1 as well as Propositions 6.5 and 6.6).

(d) $F$ and $G$ can be nonlocal maps possessing the Volterra property.

(e) The negative Laplace operator $-\Delta$ and $\partial_{\nu}$ can be replaced by a general second order strongly uniformly elliptic operator in divergence form and by the corresponding conormal derivative, respectively. Furthermore, the above results extend to systems and higher order parabolic boundary value problems.

(f) Problems (0.3) and (0.7) enjoy important comparison theorems (see Theorems [5.3, 5.5, 6.3, and (7.3).

Theorem 0.1 is the first result taking into consideration measure data depending nonlinearly on the solution. So far, almost all papers in this field deal with very particular model problems in which $\vec{\mu}$ is independent of $u$ (see the references in Sections 5 [8).

In order to illustrate the scope of Theorem 0.1 (and its generalizations) we now present two model problems, where, for simplicity, we assume that $\Omega$ is bounded. First we suppose that

$$
X: \mathbb{R}^{+} \rightarrow \Omega, \quad Y: \mathbb{R}^{+} \rightarrow \Gamma_{1}
$$

are locally Lipschitz continuous maps. Then, given $v \in L_{1}\left(J, L_{1}(\Omega)\right)$, we put

$$
x(v, t):=X\left(\int_{0}^{t} \int_{\Omega^{*}} v(x, \tau) d x d \tau\right), \quad y(v, t):=Y\left(\int_{0}^{t} \int_{\Omega^{\prime}} v(x, \tau) d x d \tau\right)
$$


for $t \in J$, where $\Omega^{*}$ and $\Omega^{\prime}$ are nonempty open subsets of $\Omega$. We also assume that $\varphi, \psi \in \mathcal{M}_{\mathrm{loc}}\left(\mathbb{R}^{+}\right)$and $\left(\mu_{\Omega}, \mu_{0}, \mu_{1}\right) \in \mathcal{M}_{\mathrm{loc}}\left(\mathbb{R}^{+}, \mathcal{M}(\Omega) \times \mathcal{M}\left(\Gamma_{0}\right) \times \mathcal{M}\left(\Gamma_{1}\right)\right)$, as well as $a \in L_{\infty}$,loc $\left(\mathbb{R}^{+}, L_{\infty}(\Omega)\right)$, are nonnegative. Then we consider the nonlinear heat equation

$$
\begin{aligned}
& \partial_{t} u-\Delta u=-a u^{\lambda}+\delta_{x(u, t)} \otimes \varphi+\mu_{\Omega} \quad \text { in } \quad \Omega \times \mathbb{R}^{+}, \\
& u=\mu_{0} \quad \text { on } \Gamma_{0} \times \mathbb{R}^{+}, \\
& \partial_{\nu} u=\delta_{y(u, t)} \otimes \psi+\mu_{1} \quad \text { on } \quad \Gamma_{1} \times \mathbb{R}^{+} .
\end{aligned}
$$

Note that this problem contains heat sources located at points $x(u, t) \in \Omega$ and $y(u, t) \in \Gamma_{1}$ whose positions at time $t$ depend on the total heat in $\Omega^{*} \times[0, t)$ and $\Omega^{\prime} \times[0, t)$, respectively. In addition, if $\varphi$ and $\psi$ vanish on some intervals, then these points disappear and may reappear again.

Theorem 0.3. Let the above condition be satisfied and suppose that

$$
1<\lambda<(n+2) /(n+1) \text {. }
$$

Then problem (0.10) possesses a unique global positive $L_{\lambda}\left(L_{\lambda}\right)$-solution.

Proof. Put

$$
\vec{\mu}_{*}(v):=\left(\delta_{x(v, \cdot)} \otimes \varphi+\mu_{\Omega}, \mu_{0}, \delta_{y(v, \cdot)} \otimes \psi+\mu_{1}\right) .
$$

One verifies that $v \mapsto \vec{\mu}_{*}(v)$ is locally Lipschitz continuous from $L_{\lambda}\left((0, T), L_{\lambda}(\Omega)\right)$ into $\mathcal{M}\left([0, T], \mathcal{M}^{-1}(\Omega) \times \mathcal{M}\left(\Gamma_{0}\right) \times \mathcal{M}^{-1}\left(\Gamma_{1}\right)\right)$ for every $T>0$, and that it has the Volterra property. Thus (0.10) is a special case of (0.3) in the generalized version of Remark $0.2(\mathrm{a})$. From this we obtain the existence of a maximal $L_{\lambda}\left(L_{\lambda}\right)$-solution (cf. the proof of Proposition 5.16 and Remark 5.17(a)). Positivity is a consequence of Corollary 5.4, and global existence is derived as in the proof of Theorem 5.14

Clearly, Theorem 0.3 can easily be generalized to the case of several heat sources in $\Omega$ and on $\Gamma_{1}$. In Section 5 we consider the case where the positions of the heat sources at time $t$ depend on the average heat $\int_{\Omega_{j}} u(x, t) d x$ at time $t$ (see Proposition 5.16).

Our next model problem deals with moving interfaces. For simplicity, we consider a very simple geometry. Much more general situations are studied in Section 5 .

We suppose that

$$
X: \mathbb{R}^{+} \rightarrow \mathbb{R}^{n}, \quad R, \mathrm{~A}: \mathbb{R}^{+} \rightarrow \mathbb{R}^{+}
$$

are locally Lipschitz continuous maps and $\mathrm{A}$ is bounded. Then, given $v \in L_{1}(\Omega)$ with $v \geq 0$, we put

$$
x(v):=X\left(\int_{\Omega} v d x\right), \quad r(v):=R\left(\int_{\Omega} v d x\right), \quad \alpha(v):=\mathrm{A}\left(\int_{\Omega} v d x\right) .
$$

We also set

$$
M(v):= \begin{cases}\{x \in \Omega ;|x-x(v)|=r(v)\} & \text { if } r(v)>0 \\ \emptyset & \text { otherwise. }\end{cases}
$$

Thus $M(v)$ is the intersection of $\Omega$ with the $(n-1)$-sphere with radius $r(v)$ and center at $x(v)$, if $r(v)>0$. We also denote by $\boldsymbol{n}(v)$ the outer unit normal of $M(v)$, that is, $\boldsymbol{n}(v)(x):=(x-x(v)) / r(v)$ for $x \in M(v)$, if $M(v) \neq \emptyset$. Then $M(v)$ is a 
smooth hypersurface of $\Omega$ oriented by $\boldsymbol{n}(v)$. Thus, given a piecewise smooth function $u$, the jump of its normal derivative along $M(v)$, denoted by $\left[\partial_{\boldsymbol{n}(v)} u\right]_{M(v)}$, has the obvious meaning (see Section 5 for precise definitions).

We assume that $a_{0} \in L_{\infty, \text { loc }}\left(\mathbb{R}^{+}, L_{\infty}(\Omega)\right)$ and

$$
\left(\mu_{\Omega}, \mu_{0}, \mu_{1}\right) \in L_{\infty, \text { loc }}\left(\mathbb{R}^{+}, \mathcal{M}(\Omega) \times \mathcal{M}\left(\Gamma_{0}\right) \times \mathcal{M}\left(\Gamma_{1}\right)\right)
$$

are nonnegative. Then we consider the following initial boundary value problem with a transmission condition:

$$
\begin{array}{rlrlrl}
\partial_{t} u-\Delta u & =-a u^{\lambda}+\mu_{\Omega} & & \text { in } & & \Omega \times(0, \infty), \\
{\left[\partial_{\boldsymbol{n}(u)} u\right]_{M(u)}} & =\alpha(u) & & \text { on } & & M(u), \\
u & =\mu_{0} & & \text { on } & \Gamma_{0} \times(0, \infty), \\
\partial_{\nu} u & =\mu_{1} & & \text { on } & \Gamma_{1} \times(0, \infty), \\
u(\cdot, 0) & =u^{0} & & \text { on } & & \Omega .
\end{array}
$$

Clearly, the second equation means that, at time $t$, the jump of the normal derivative of the solutions $u(t)$ along $M(u(t))$ has the value $\alpha(u(t))$. Note that $\{M(u(t)) ; t \in J(u)\}$ is a family of moving interfaces which may disappear and reappear, depending on the mean value $\int_{\Omega} u(x, t) d x$ of the solution $u$ at time $t$. Moreover, the size of the jump of its normal derivative along $M(u(t))$ depends on this mean value as well.

By a positive (weak) $C\left(L_{\lambda}\right)$-solution of (0.11) on $J$ we mean a nonnegative function $u \in C\left(J, L_{\lambda}(\Omega)\right)$ satisfying

$$
\begin{aligned}
& \int_{J} \int_{\Omega}(\left.-\partial_{t} \varphi-\Delta \varphi\right) u d x d t \\
&=\int_{J}\left\{\int_{\Omega}-\varphi(t) a(t) u^{\lambda}(t) d x+\int_{\Omega} \varphi(t) d \mu_{\Omega}(t)-\int_{\Gamma_{0}} \partial_{\nu} \varphi(t) d \mu_{0}(t)\right. \\
&\left.\quad+\int_{\Gamma_{1}} \varphi(t) d \mu_{1}(t)+\int_{M(u(t))} \varphi(t) \alpha(u(t)) d \operatorname{vol}_{M(u(t))}\right\} d t \\
& \quad+\int_{\Omega} \varphi(0) u^{0} d x
\end{aligned}
$$

for each $\varphi \in \mathcal{D}\left(\bar{\Omega} \times J^{*}\right)$ satisfying $(\overline{0.6})$.

The following theorem is a particular case of the much more general Corollary 5.13 and Theorem 5.14 .

Theorem 0.4. Suppose that $1<\lambda<n /(n-1)$. Then problem (0.11) has for each $u^{0} \in W_{\lambda}^{1-n / \lambda^{\prime}}$ with $u^{0} \geq 0$ a unique global positive $C\left(L_{\lambda}\right)$-solution.

This paper consists of four parts and an appendix. In Part 1 we present the abstract results for problem (0.1). Their proofs are deferred to Part 4 . The second part illustrates the power of the general theorems by means of applications to a variety of model problems. In Part 3 we extend the foregoing results to systems. In the appendix we provide mapping properties of Nemytskii operators possessing only a little regularity, which are needed in the applications of Part 2 and are of independent interest. Then we study linear problems, thus complementing [5] by extending those results to nonautonomous equations and giving compactness theorems. Nonautonomous equations occur in the proof of a version of a weak 
maximum principle which is somewhat technical and therefore relegated to this appendix. However, it is crucial for the positivity and comparison theorems mentioned above.

It should be noted that the results of Sections 57 generalize earlier investigations of other authors considerably. We refer to these sections for discussions of the relation between our work and those earlier results. However, we emphasize that this paper contains the first systematic study of general parabolic evolution equations involving measures which depend nonlinearly on the solution.

Finally, we direct the readers' attention towards Remark 4.2(c) for a correction and improvement of an erroneous assertion made in [5].

\section{Part 1. The Abstract Results}

Throughout all of this part we assume that

- $E_{0}$ is a reflexive Banach space;

- $A_{0}$ is the negative infinitesimal generator of a strongly continuous analytic semigroup on $E_{0}$.

We put $E_{1}:=D\left(A_{0}\right)$, where $D\left(A_{0}\right)$ is the domain of $A_{0}$ endowed with the graph norm. We also fix a real number $\omega_{0}>0$ such that the resolvent set $\omega_{0}+A_{0}$ contains the left complex half-plane $[\operatorname{Re} z \leq 0]$ and denote by $E_{-1}$ the completion of $E_{0}$ with respect to the norm $u \mapsto\left\|\left(\omega_{0}+A\right)^{-1} u\right\|_{E_{0}}$. Then $E_{1}$ and $E_{-1}$ are Banach spaces satisfying

$$
E_{1} \stackrel{d}{\hookrightarrow} E_{0} \stackrel{d}{\hookrightarrow} E_{-1},
$$

where $\hookrightarrow$ denotes "continuous injection" and the superscript $d$ means "dense". Moreover, $E_{-1}$ is independent of the choice of $\omega_{0}$, except for equivalent norms.

For each $\theta \in(0,1)$ we fix an admissible interpolation functor of exponent $\theta$, where "admissible" means that either $(\cdot, \cdot)_{\theta}$ equals the real interpolation functor $(\cdot, \cdot)_{\theta, q}$ for some $q \in(1, \infty)$, or $(\cdot, \cdot)_{\theta}$ is the complex interpolation functor $[\cdot, \cdot]_{\theta}$. Then we set

$$
E_{k+\theta}:=\left(E_{k}, E_{k+1}\right)_{\theta}, \quad 0<\theta<1, \quad k \in\{-1,0\} .
$$

It follows that

$$
E_{s} \stackrel{d}{\hookrightarrow} E_{t}, \quad-1 \leq t<s \leq 1
$$

Furthermore, there exists a unique $A_{-1} \in \mathcal{L}\left(E_{0}, E_{-1}\right)$ extending $A_{0}$, where $\mathcal{L}$ is the space of bounded linear operators. We denote by $A_{s}$ the maximal restriction of $A_{-1}$ to $E_{s}$ for $1<s \leq 1$. Then

$$
A_{t} \supset A_{s}, \quad-1 \leq t<s \leq 1,
$$

the operator $-A_{s}$ generates a strongly continuous analytic semigroup on $E_{s}$ for $-1 \leq s \leq 1$, and $A_{s} \in \mathcal{L}\left(E_{s+1}, E_{s}\right)$ for $-1 \leq s \leq 0$. The family

$$
\left[\left(E_{s}, A_{s}\right) ;-1 \leq s \leq 1\right]
$$

is said to be the interpolation extrapolation scale generated by $\left(E_{0}, A_{0}\right)$ and $(\cdot, \cdot)_{\theta}$, $0<\theta<1$. For proof of these facts and more details we refer to [3, Chapter V].

Now we can formulate the second basic hypothesis which is presupposed throughout Part 1. Namely, we assume that

- $0 \leq \alpha<\beta<1$;

- $A:=A_{\alpha-1}$. 
We also fix a positive real number $\mathrm{T}$ and consider the semilinear evolution equation

$$
\dot{u}+A u=\mu(u) \quad \text { in }[0, T],
$$

where $\mu$ is an $E_{\beta-1}$-valued measure on $[0, \mathrm{~T}]$ depending nonlinearly on $u$ and possessing the Volterra property.

Equation (EV) is a suitably generalized formulation of the Cauchy problem $\dot{u}+A_{0} u=\mu(u)$ which, in general, is not well-posed. This will be clear by looking at the concrete applications of the abstract theory presented in Part 2 Note that $A \in \mathcal{L}\left(E_{\alpha}, E_{\alpha-1}\right)$, so that $(\mathrm{EV})$ is an equation in $E_{\alpha-1}$. The hypothesis that $\mu(u)$ be an $E_{\beta-1}$-valued measure is a regularity condition meaning that the measure is "subordinate" to the linear operator $A$.

\section{WEAK SOLUTIONS OF SEMILINEAR PROBLEMS}

In this first section we consider a setting in which solutions to (EV) cannot be expected to have a time derivative in a strong sense. Thus (EV) has to be interpreted in a generalized sense. For this we need some preparation.

Denote by $E_{0}^{\sharp}$ and $A_{0}^{\sharp}$ the dual of $E_{0}$ and $A_{0}$, respectively. Then $E_{0}^{\sharp}$ is a reflexive Banach space as well, and $-A_{0}^{\sharp}$ generates a strongly continuous analytic semigroup on $E_{0}^{\sharp}$. Hence we can define $E_{1}^{\sharp}$ and $E_{-1}^{\sharp}$ by replacing in the definition of $E_{1}$ and $E_{-1}$ the space $E_{0}$ by $E_{0}^{\sharp}$ and the operator $A_{0}$ by $A_{0}^{\sharp}$. We also set

$$
E_{k+\theta}^{\sharp}:=\left(E_{k}, E_{k+1}\right)_{\theta}^{\sharp}, \quad 0<\theta<1, \quad k \in\{-1,0\},
$$

where $(\cdot, \cdot)_{\theta}^{\sharp}$ is the interpolation functor dual to $(\cdot, \cdot)_{\theta}$. Then

$$
E_{s}^{\sharp} \stackrel{d}{\hookrightarrow} E_{t}^{\sharp}, \quad-1 \leq t<s \leq 1,
$$

and there exists a unique $A_{-1}^{\sharp} \in \mathcal{L}\left(E_{0}^{\sharp}, E_{-1}^{\sharp}\right)$ extending $A_{0}^{\sharp}$. We let $A_{s}^{\sharp}$ be the maximal restriction of $A_{-1}^{\sharp}$ to $E_{s}^{\sharp}$ for $-1<s \leq 1$. It is a basic fact (cf. [3, Theorem V.1.5.12]) that

$$
\left(E_{s}\right)^{\prime}=E_{-s}^{\sharp}, \quad\left(A_{s}\right)^{\prime}=A_{-s}^{\sharp}, \quad-1 \leq s \leq 1,
$$

with respect to the $E_{s}$-duality pairing $\langle\cdot, \cdot\rangle_{s}$, naturally induced by the $E_{0}$-duality pairing. Henceforth,

$$
A^{\top}:=A_{-\alpha}^{\sharp} .
$$

It follows that $A^{\top} \in \mathcal{L}\left(E_{1-\alpha}^{\sharp}, E_{-\alpha}^{\sharp}\right)$ and that it is the dual of the bounded linear operator $A_{\alpha} \in \mathcal{L}\left(E_{\alpha}, E_{\alpha-1}\right)$.

We denote by $\mathcal{M}\left([0, T], E_{\beta-1}\right)$ the Banach space of all $E_{\beta-1}$-valued Radon measures of bounded variation on $[0, T]$. By the generalized Riesz representation theorem and (1.1) it is the dual space of $C\left([0, T], E_{1-\beta}^{\sharp}\right)$. Thus we also write suggestively

$$
\int_{[0, \mathrm{~T}]} v d \mu, \quad(\mu, v) \in \mathcal{M}\left([0, \mathrm{~T}], E_{\beta-1}\right) \times C\left([0, \mathrm{~T}], E_{1-\beta}^{\sharp}\right),
$$

for the corresponding duality pairing.

Suppose that $1<p<\infty$, that

$$
\mu: L_{p}\left((0, T), E_{\alpha}\right) \rightarrow \mathcal{M}\left([0, \mathrm{~T}], E_{\beta-1}\right),
$$

and that it possesses the Volterra property. Let $J$ be a subinterval of $[0, \mathrm{~T}]$ containing 0 and more than one point (i.e., a perfect interval). Recall that $J^{*}:=J \backslash\{\sup J\}$. 
By a weak $L_{p}\left(E_{\alpha}\right)$-solution of $(\mathrm{EV})$ on $J$ we mean a $u \in L_{p, \text { loc }}\left(J^{*}, E_{\alpha}\right)$ satisfying

$$
\int_{J}\left\langle-\dot{v}+A^{\top} v, u\right\rangle_{\alpha} d t=\int_{J} v d \mu(u)
$$

for all $v \in C_{c}\left(J^{*}, E_{1-\alpha}^{\sharp}\right) \cap C^{1}\left(J^{*}, E_{-\alpha}^{\sharp}\right)$, where the subscript $c$ means "compact support". Note that (1.2) is meaningful since $E_{1-\alpha}^{\sharp} \hookrightarrow E_{1-\beta}^{\sharp}$ and $v$ vanishes near the right endpoint of $J$, so that, given $T \in J$ with $v(t)=0$ for $t \geq T$, the Volterra property implies

$$
\int_{J} v d \mu(u)=\int_{[0, \mathrm{~T}]} v d \mu(u)
$$

A weak $L_{p}\left(E_{\alpha}\right)$-solution $u$ is maximal if there does not exist another such solution being a proper extension of it. Then $J(u):=\operatorname{dom}(u)$ is its maximal interval of existence. A weak $L_{p}\left(E_{\alpha}\right)$-solution $u$ is global if $J(u)=[0, T]$ and $u \in L_{p}\left((0, T), E_{\alpha}\right)$. In this case [5, Corollary 2] guarantees that $u$ has a well-defined trace $u(\mathrm{~T})$ at $\mathrm{T}$ in $E_{\beta-1}$. (Notice that, choosing $x \in E_{\beta-1}$, any weak solution of $(\mathrm{EV})$ on $[0, \mathrm{~T}]$ is also a weak solution of the equation

$$
\dot{u}+A u=\mu(u)+x \otimes \delta_{\mathrm{T}} \quad \text { on }[0, \mathrm{~T}],
$$

due to the fact that every test function $v$ in (1.2) vanishes near $\mathrm{T}$. The trace theorem eliminates this ambiguity.)

Let $F$ and $G$ be normed vector spaces. Then $C_{b}^{1-}(F, G)$ is the space of all maps from $F$ into $G$ which are bounded on bounded sets and uniformly Lipschitz continuous on such sets.

Now we can formulate our general existence and uniqueness theorem for maximal weak $L_{p}\left(E_{\alpha}\right)$-solutions.

Theorem 1.1. Suppose that $1<p<1 /(\alpha-\beta+1)$ and that

$$
\mu \in C_{b}^{1-}\left(L_{p}\left((0, \mathrm{~T}), E_{\alpha}\right), \mathcal{M}\left([0, \mathrm{~T}], E_{\beta-1}\right)\right)
$$

has the Volterra property. Also suppose that there exists an admissible interpolation functor $\{\cdot, \cdot\}_{\beta-\alpha}$ of exponent $\beta-\alpha$ such that

$$
E_{\beta-1} \doteq\left\{E_{\alpha-1}, E_{\alpha}\right\}_{\beta-\alpha} .
$$

Then:

(i) There exists a unique maximal weak $L_{p}\left(E_{\alpha}\right)$-solution $u:=u(\mu)$ of $(\mathrm{EV})$.

(ii) If $u \in L_{p}\left(J(u), E_{\alpha}\right)$, then $u$ is global.

It is an important additional fact that the solution $u(\mu)$ depends Lipschitz continuously on $\mu$. The precise assertion is formulated in the next theorem. Note that assumption (1.3) implies the existence of a constant $\omega$ with

$$
\|\mu(0)\|_{\mathcal{M}\left([0, \mathrm{~T}], E_{\beta-1}\right)} \leq \omega
$$

and of an increasing function $\lambda: \mathbb{R}^{+} \rightarrow \mathbb{R}^{+}$satisfying

$$
\|\mu(v)-\mu(w)\|_{\mathcal{M}\left([0, \mathrm{~T}], E_{\beta-1}\right)} \leq \lambda(R)\|v-w\|_{L_{p}\left((0, \mathrm{~T}), E_{\alpha}\right)}
$$

for each $R>0$ and all $v, w \in L_{p}\left((0, \mathrm{~T}), E_{\alpha}\right)$ whose norms are bounded by $R$. 
Theorem 1.2. Let the hypotheses of Theorem 1.1 be satisfied. Fix any positive $T \in J(u(\mu))$, where $T<\sup (J(u(\mu)))$ if $u(\mu)$ is not global. Also fix $\omega$ and $\lambda$ satisfying (1.5) and (1.6), respectively, as well as $R$ with $R>\|u(\mu)\|_{L_{p}\left((0, T), E_{\alpha}\right)}$. Then there exists $\varepsilon>0$ such that $J(u(\nu)) \supset[0, T]$ whenever

$$
\nu \in C_{b}^{1-}\left(L_{p}\left((0, \mathrm{~T}), E_{\alpha}\right), \mathcal{M}\left([0, \mathrm{~T}], E_{\beta}\right)\right)
$$

has the Volterra property and satisfies (1.5) and (1.6) as well as

$$
\sup _{\|v\|_{L_{p}\left((0, \mathrm{~T}), E_{\alpha}\right) \leq R}}\|(\nu-\mu)(v)\|_{\mathcal{M}\left([0, T], E_{\beta-1}\right)} \leq \varepsilon .
$$

Moreover, there exists a constant $c$ such that

$$
\|u(\nu)-u(\widetilde{\nu})\|_{L_{p}\left((0, T), E_{\alpha}\right)} \leq c \sup _{\|v\|_{L_{p}\left((0, T), E_{\alpha}\right) \leq R}}\|(\nu-\widetilde{\nu})(v)\|_{\mathcal{M}\left([0, T], E_{\beta-1}\right)}
$$

for all $\nu, \widetilde{\nu} \in C_{b}^{1-}\left(L_{p}\left((0, \mathrm{~T}), E_{\alpha}\right), \mathcal{M}\left([0, \mathrm{~T}], E_{\beta-1}\right)\right)$ possessing the Volterra property and satisfying estimates (1.5)-(1.7).

The constants $\varepsilon$ and $c$ depend on $R, T, \lambda$, and $\omega$ only.

The proofs of these theorems are postponed to Section [13.

\section{Strong SOlutions of SEMiLinear PRoblems}

In this section we present existence, uniqueness, and continuity theorems for $(\mathrm{EV})$ in cases where $\mu(u)$ is absolutely continuous with respect to Lebesgue's measure on $[0, T]$ and satisfies suitable integrability conditions.

Let $J$ be a perfect subinterval of $[0, T]$ containing 0 . Given $\gamma \in[0,1]$, put

$$
\mathbb{W}_{p, \text { loc }}^{1}\left(J^{*},\left(E_{\gamma-1}, E_{\gamma}\right)\right):=L_{p, \text { loc }}\left(J^{*}, E_{\gamma}\right) \cap W_{p, \text { loc }}^{1}\left(J^{*}, E_{\gamma-1}\right), \quad 1 \leq p \leq \infty .
$$

Recall that the trace theorem implies

$$
\mathbb{W}_{p, \text { loc }}^{1}\left(J^{*},\left(E_{\gamma-1}, E_{\gamma}\right)\right) \hookrightarrow C\left(J^{*},\left(E_{\gamma-1}, E_{\gamma}\right)_{1 / p^{\prime}, p}\right), \quad 1<p<\infty
$$

(cf. [3, Theorem III.4.10.2]).

We assume that

$$
1<r<1 /(\beta-\alpha) \text { and } 1 \leq p<1 /(\alpha-\beta+1 / r) .
$$

We also assume that

$$
f: L_{p}\left((0, \mathrm{~T}), E_{\alpha}\right) \rightarrow L_{r}\left((0, \mathrm{~T}), E_{\beta-1}\right)
$$

and that it has the Volterra property. Then, given $x \in E_{\beta-1}$, we consider the semilinear initial value problem

$$
\dot{u}+A u=f(u) \text { in }(0, \mathrm{~T}], \quad u(0)=x .
$$

By a strong $L_{r}\left(E_{\gamma}\right)$-solution of (2.2) on $J$, where $\alpha \leq \gamma<\beta$, we mean an element

$$
u \in L_{p, \operatorname{loc}}\left(J^{*}, E_{\alpha}\right) \cap \mathbb{W}_{r, \text { loc }}^{1}\left(J^{*},\left(E_{\gamma-1}, E_{\gamma}\right)\right)
$$

such that $u$ satisfies (2.2) in the obvious (that is, pointwise a.e.) sense (cf. [3. Theorem III.1.2.2]). Thanks to the Volterra property this definition is meaningful, and (2.1) implies that $u \in C\left(J^{*}, E_{\gamma-1}\right)$, so that the initial condition is also welldefined. Maximal and global strong solutions are defined similarly as for weak solutions.

Due to [5, Theorem 3], $\mathbb{W}_{r, \text { loc }}^{1}\left(J^{*},\left(E_{\gamma-1}, E_{\gamma}\right)\right) \hookrightarrow L_{p, \text { loc }}\left(J^{*}, E_{\alpha}\right)$ if $\gamma$ is close to $\beta$; hence the assumption $u \in L_{p, \text { loc }}\left(J^{*}, E_{\alpha}\right)$ is automatically satisfied in this 
case. Moreover, the definition of $L_{r}\left(E_{\gamma}\right)$-solutions does not depend on $\gamma$ : if $u$ is an $L_{r}\left(E_{\gamma}\right)$-solution for some $\gamma \in[\alpha, \beta)$, then $f(u) \in L_{r, \text { loc }}\left(J(u), E_{\beta-1}\right)$ and [5, Theorem 5] guarantees $u \in \mathbb{W}_{r, \text { loc }}^{1}\left(J(u),\left(E_{\gamma-1}, E_{\gamma}\right)\right)$ for any $\gamma<\beta$. Hence $u$ is an $L_{r}\left(E_{\gamma}\right)$-solution for any $\gamma \in[\alpha, \beta)$.

Using these notations and definitions, we can formulate the following existence, uniqueness, and continuity theorem for maximal strong $L_{r}\left(E_{\gamma}\right)$-solutions.

Theorem 2.1. Assume that $1<r<1 /(\beta-\alpha)$ and $1 \leq p<1 /(\alpha-\beta+1 / r)$. Also suppose that $\alpha \leq \gamma<\beta$, that

$$
(x, f) \in E_{\beta-1 / r} \times C_{b}^{1-}\left(L_{p}\left((0, \mathrm{~T}), E_{\alpha}\right), L_{r}\left((0, \mathrm{~T}), E_{\beta-1}\right)\right),
$$

and that $f$ has the Volterra property. Then:

(i) Problem (2.2) has a unique maximal strong $L_{r}\left(E_{\gamma}\right)$-solution $u:=u(x, f)$.

(ii) If $u \in L_{p}\left(J(u), E_{\alpha}\right)$, then $u$ is global.

As before, an important continuity theorem is valid in this case also. For this we observe that assumption (2.3) implies the existence of a constant $\omega$ such that

$$
\|x\|_{E_{\beta-1 / r}}+\|f(0)\|_{L_{r}\left((0, \mathrm{~T}), E_{\beta-1}\right)} \leq \omega
$$

and of an increasing function $\lambda: \mathbb{R}^{+} \rightarrow \mathbb{R}^{+}$satisfying

$$
\|f(u)-f(v)\|_{L_{r}\left((0, \mathrm{~T}), E_{\beta-1}\right)} \leq \lambda(R)\|u-v\|_{L_{p}\left((0, \mathrm{~T}), E_{\alpha}\right)}
$$

for each $R>0$ and all $u, v \in L_{p}\left((0, T), E_{\alpha}\right)$ whose norms are bounded by $R$.

Theorem 2.2. Let the hypotheses of Theorem 2.1 be satisfied. Fix any positive $T \in J(u(x, f))$, where $T<\sup (J(x, f))$ if $u(x, f)$ is not global. Also fix $\omega$ and $\lambda$ satisfying (2.4) and (2.5), respectively, as well as $R>\|u(x, f)\|_{L_{p}\left((0, T), E_{\alpha}\right)}$. Then there exists $\varepsilon>0$ such that $J(u(y, g)) \supset[0, T]$ whenever

$$
(y, g) \in E_{\beta-1 / r} \times C_{b}^{1-}\left(L_{p}\left((0, \mathrm{~T}), E_{\alpha}\right), L_{r}\left((0, \mathrm{~T}), E_{\beta-1}\right)\right)
$$

are such that $g$ possesses the Volterra property, $(y, g)$ satisfies (2.4) and (2.5), and

$$
\|y-x\|_{E_{\beta-1 / r}}+\sup _{\|v\|_{L_{p}\left((0, \mathrm{~T}), E_{\alpha}\right) \leq R}}\|(g-f)(v)\|_{L_{r}\left((0, \mathrm{~T}), E_{\beta-1}\right)} \leq \varepsilon .
$$

Moreover, there exists a constant $c$ such that

$$
\begin{aligned}
\| u(y, g) & -u(\widetilde{y}, \widetilde{g}) \|_{\mathbb{W}_{r}^{1}\left((0, T),\left(E_{\gamma-1}, E_{\gamma}\right)\right)} \\
& \leq c\left(\|y-\widetilde{y}\|_{E_{\beta-1 / r}}+\sup _{\|v\|_{L_{p}\left((0, \mathrm{~T}), E_{\alpha}\right) \leq R}}\|(g-\widetilde{g})(v)\|_{L_{r}\left((0, \mathrm{~T}), E_{\beta-1}\right)}\right)
\end{aligned}
$$

for all $(y, \widetilde{y}) \in E_{\beta-1 / r}$ and $g, \widetilde{g} \in C_{b}^{1-}\left(L_{p}\left((0, T), E_{\alpha}\right), L_{r}\left((0, T), E_{\beta-1}\right)\right)$ possessing the Volterra property and satisfying (2.4)-2.6). The constants $\varepsilon$ and $c$ depend on $R, T, \lambda$, and $\omega$ only.

Next we turn to the case where $r>1 /(\beta-\alpha)$. As usual, $C^{\rho}$ denotes the space of (locally) $\rho$-Hölder continuous maps.

Theorem 2.3. Suppose that $1 /(\beta-\alpha)<r<\infty$ and $0 \leq \rho<\beta-\alpha-1 / r$. Then Theorems 2.1 and 2.2 remain valid if we replace the spaces $L_{p}\left((0, \mathrm{~T}), E_{\alpha}\right)$ and $L_{p}\left((0, T), E_{\alpha}\right)$ by $C^{\rho}\left([0, T], E_{\alpha}\right)$ and $C^{\rho}\left([0, T], E_{\alpha}\right)$, respectively.

We close this section with a series of remarks containing useful complements and extensions of the preceding general theorems. 
Remarks 2.4. (a) Given the hypotheses of Theorem[1.1, the maximal solution $u(\mu)$ is global iff the $E_{\beta-1}$-valued measure $\mu(u(\mu))$ is of bounded variation on $J(u(\mu))$. Similarly, given the hypotheses of either Theorem [2.1] or [2.3, the maximal solution $u(x, f)$ is global iff $f(u(x, f))$ belongs to $L_{r}\left(J(u(x, f)), E_{\beta-1}\right)$.

(b) Let the hypotheses of Theorem 1.1 be satisfied. Also suppose that $\mu$ is linearly bounded, that is,

$$
\|\mu(v)\|_{\mathcal{M}\left((0, \mathbf{T}), E_{\beta-1}\right)} \leq c\left(1+\|v\|_{L_{p}\left((0, \mathbf{T}), E_{\alpha}\right)}\right)
$$

for all $v \in L_{p}\left((0, \mathrm{~T}), E_{\alpha}\right)$. Then $\mu(u)$ is global. Similarly, let the assumptions of Theorem 2.1 be valid and suppose that

$$
\|f(v)\|_{L_{r}\left((0, \mathbf{T}), E_{\beta-1}\right)} \leq c\left(1+\|v\|_{L_{p}\left((0, \mathbf{T}), E_{\alpha}\right)}\right)
$$

for all $v \in L_{p}\left((0, \mathrm{~T}), E_{\alpha}\right)$. Then the strong solution $u(x, f)$ of (2.2) is global. If the hypotheses of Theorem 2.3 are satisfied and (2.7) holds with $L_{p}\left((0, T), E_{\alpha}\right)$ replaced by $C^{\rho}\left([0, \mathrm{~T}], E_{\alpha}\right)$, then the corresponding strong solution $u(x, f)$ exists on $[0, T]$ as well.

(c) The assumptions of Theorem 2.1 imply that $u(x, f) \in C\left(J(u(x, f)), E_{\gamma-1 / r}\right)$.

(d) Assume that $\alpha_{j}<\beta<\alpha_{j}+1$ and $1<p_{j}<\left(\alpha_{j}-\beta+1\right)$ for $j=0,1, \ldots, m$. Set

$$
L_{\vec{p}}\left((0, T), E_{\vec{\alpha}}\right):=\bigcap_{j=0}^{m} L_{p_{j}}\left((0, T), E_{\alpha_{j}}\right), \quad 0<T \leq \mathrm{T} .
$$

Also suppose that $\mu: L_{\vec{p}}\left((0, \mathrm{~T}), E_{\vec{\alpha}}\right) \rightarrow \mathcal{M}\left([0, \mathrm{~T}], E_{\beta-1}\right)$ has the Volterra property. Then by a weak $L_{\vec{p}}\left(E_{\vec{\alpha}}\right)$-solution of $(\mathrm{EV})$ on $J$ we mean an element

$$
u \in \bigcap_{j=0}^{m} L_{p_{j}, \text { loc }}\left(J^{*}, E_{\alpha_{j}}\right)=: L_{\vec{p}, \text { loc }}\left(J^{*}, E_{\vec{\alpha}}\right)
$$

satisfying (1.2) for each $\alpha_{j}$ and each $v \in C_{c}\left(J^{*}, E_{1-\alpha_{j}}^{\sharp}\right) \cap C^{1}\left(J^{*}, E_{-\alpha_{j}}^{\sharp}\right)$.

Replace $L_{p}\left((0, \mathrm{~T}), E_{\alpha}\right)$ and $L_{p}\left((0, T), E_{\alpha}\right)$ in Theorems 1.1 and 1.2 as well as in (1.6) by $L_{\vec{p}}\left((0, T), E_{\vec{\alpha}}\right)$ and $L_{\vec{p}}\left((0, T), E_{\vec{\alpha}}\right)$, respectively. Then these theorems remain valid and guarantee the existence of a unique maximal weak $L_{\vec{p}}\left(E_{\vec{\alpha}}\right)$-solution with the corresponding continuity and global existence properties, provided for each $j$ there exists an admissible interpolation functor $\{\cdot, \cdot\}_{\beta-\alpha_{j}}$ of exponent $\beta-\alpha_{j}$ such that $E_{\beta-1} \doteq\left\{E_{\alpha_{j}-1}, E_{\alpha_{j}}\right\}_{\beta-\alpha_{j}}$.

Analogous extensions of Theorems 2.1, 2.2, and 2.3 are valid as well.

(e) Let the assumptions of Theorem[1.1 be satisfied for $\mu \in\left\{\mu_{1}, \mu_{2}\right\}$. Assume, in addition, that $E_{0}$ is an ordered Banach space, $A_{0}$ is resolvent positive, and $u_{1} \geq u_{2}$ implies $\mu_{1}\left(u_{1}\right) \geq \mu_{2}\left(u_{2}\right)$. Then $u\left(\mu_{1}\right) \geq u\left(\mu_{2}\right)$ on $J\left(u\left(\mu_{1}\right)\right) \cap J\left(u\left(\mu_{2}\right)\right)$.

(f) We denote by $\{U(t) ; t \geq 0\}$ the semigroup generated by $-A$ on $E_{\alpha-1}$. For $0<T \leq \mathrm{T}$ and $h \in L_{1}\left((0, T), E_{\beta-1}\right)$ we set

$$
U \star h(t):=\int_{0}^{t} U(t-\tau) h(\tau) d \tau, \quad 0 \leq t \leq T .
$$

A solution of the integral equation $u=U x+U \star f(u)$ is often said to be a mild solution of (2.2). Hence Theorem 2.1)implies the existence and uniqueness of mild solutions. However, this theorem contains more detailed information. In particular, it shows that these solutions are strong ones.

The proofs of the preceding theorems and remarks are given in Section 14 


\section{Part 2. Parabolic Differential Equations}

In this part we exhibit applications of the general results presented in Part 1 to a variety of concrete parabolic initial boundary value problems. For simplicity, we restrict ourselves to second order scalar equations with smooth coefficients. Extensions to higher order problems and systems are given in Section 8 and Part 3 respectively.

More precisely, we assume throughout that

$$
\boldsymbol{a}=\left[a_{j k}\right] \in B U C^{\infty}\left(\Omega, \mathbb{R}^{n \times n}\right)
$$

is symmetric and uniformly positive definite. Then we put

$$
\mathcal{A} u:=-\nabla \cdot(\boldsymbol{a} \nabla u)
$$

and

$$
\mathcal{B} u:=\left\{\begin{aligned}
\gamma u & \text { on } \Gamma_{0}, \\
\partial_{\nu} u & \text { on } \Gamma_{1},
\end{aligned}\right.
$$

where $\gamma$ is the trace operator and $\partial_{\boldsymbol{\nu}}$ is the derivative with respect to the conormal $\boldsymbol{\nu}:=\boldsymbol{a} \nu$, with $\nu$ being the outer unit normal on $\Gamma$, provided $\Gamma \neq \emptyset$, of course.

Section 3 contains the main result for semilinear second order parabolic boundary value problems with measures, namely Theorems 3.2 3.4. They are obtained by straightforward applications of the theorems of Sections 1 and 2. The next section is devoted to the interpretation of these theorems in more classical terms.

Sections 50 - contain a variety of model problems. The first of these sections deals with the most singular setting in which we allow distributions more general than measures. Here we study, among other things, moving interface transmission problems as well as problems with moving point sources. In Section 6 we investigate semilinear boundary value problems with measures in the interior and on the Neumann boundary. In particular, we consider nonlinear boundary conditions and derive existence results under optimal growth restrictions. In Section 7 we investigate problems with integrable low regularity data.

In the last section we show, by means of simple model problems, how our general abstract results can be applied to higher order problems.

\section{SEmilinear SECOND ORDER PROBlems}

We also suppose that

$$
1<q<\infty \text { and } s \in[-2,2]
$$

We write $W_{q}^{s}:=W_{q}^{s}(\Omega)$ for the usual Sobolev-Slobodeckii spaces; hence $W_{q}^{0}=L_{q}$. Furthermore, $\mathscr{W}_{q}^{s}$ is the closure of $\mathcal{D}$ in $W_{q}^{s}$, where $\mathcal{D}:=\mathcal{D}(\Omega)$ is the space of smooth functions with compact support in $\Omega$. Then $\stackrel{\circ}{W}_{q}^{s}=W_{q}^{s}$ for all $s \in \mathbb{R}$ if $\Gamma=\emptyset$, and for $s<1 / q$ otherwise. Moreover, $W_{q}^{-s}=\left({\stackrel{\circ}{W^{\prime}}}^{s}\right)^{\prime}$ for $s \geq 0$ with respect to the $L_{q}$-duality pairing induced by

$$
\langle u, v\rangle:=\int_{\Omega} u \cdot v d x, \quad u, v \in \mathcal{D} .
$$

We also set $\mathcal{D}(\bar{\Omega}):=\left\{u \mid \bar{\Omega} ; u \in \mathcal{D}\left(\mathbb{R}^{n}\right)\right\}$, etc.

If $\Gamma=\emptyset$, then we put

$$
W_{q, \mathcal{B}}^{s}:=W_{q}^{s}, \quad s \in \mathbb{R} .
$$


Otherwise,

$$
W_{q, \mathcal{B}}^{s}:=\left\{\begin{array}{ccc}
\left\{u \in W_{q}^{s} ; \mathcal{B} u=0\right\}, & 1+1 / q<s \leq 2, \\
\left\{u \in W_{q}^{s} ; \gamma u=0 \text { on } \Gamma_{0}\right\}, & 1 / q<s<1+1 / q, \\
W_{q}^{s}, & 0 \leq s<1 / q, \\
\left(W_{q^{\prime}, \mathcal{B}}^{-s}\right)^{\prime}, & -2 \leq s<0, \quad s \notin \mathbb{Z}+1 / q,
\end{array}\right.
$$

where $\left(W_{q^{\prime}, \mathcal{B}}^{-s}\right)^{\prime}$ is determined by the duality pairing induced by (3.1). Furthermore, the values $s=1+1 / q$ and $s=-2+1 / q$ are admitted if $\Gamma=\Gamma_{0}$, and $s=1 / q$ and $s=-1+1 / q$ are included if $\Gamma=\Gamma_{1}$. Finally,

$$
I_{q}:= \begin{cases}{[-2,2],} & \Gamma=\emptyset, \\ {[-2,2] \backslash\{1 / q,-1+1 / q\},} & \Gamma=\Gamma_{0} \neq \emptyset, \\ {[-2,2] \backslash\{1+1 / q,-2+1 / q\},} & \Gamma=\Gamma_{1} \neq \emptyset, \\ {[-2,2] \backslash(\mathbb{Z}+1 / q),} & \text { otherwise. }\end{cases}
$$

We define the $L_{q}$-realization $A_{0}$ of $(\mathcal{A}, \mathcal{B})$ by

$$
\operatorname{dom}\left(A_{0}\right):=W_{q, \mathcal{B}}^{2}, \quad A_{0} u:=\mathcal{A} u .
$$

Then $A_{0}$ is densely defined in $E_{0}:=L_{q}$ and the following is true, where $\doteq$ means equal except for equivalent norms.

Lemma 3.1. (i) The operator $-A_{0}$ generates a strongly continuous analytic semigroup on $E_{0}$, and $A_{0}^{\sharp}$ is the $L_{q^{\prime}}$-realization of $(\mathcal{A}, \mathcal{B})$.

(ii) Let $\left[\left(E_{\xi}, A_{\xi}\right) ;-1 \leq \xi \leq 1\right]$ be the interpolation extrapolation scale generated by $\left(E_{0}, A_{0}\right)$ and the interpolation functors

$$
(\cdot, \cdot)_{\theta}:= \begin{cases}{[\cdot, \cdot]_{\theta}} & \text { if } \theta=1 / 2, \\ (\cdot, \cdot)_{\theta, q} & \text { if } \theta \in(0,1) \backslash\{1 / 2\} .\end{cases}
$$

Then $E_{\xi} \doteq W_{q, \mathcal{B}}^{2 \xi}$ for $2 \xi \in I_{q}$.

(iii) $E_{0}$ is an ordered Banach space with respect to the natural (pointwise) order, and $A_{0}$ is resolvent positive.

Proof. (i) and (ii) follow from [5, Theorem 6].

(iii) If $\Omega$ is bounded, this is a consequence of the maximum principle. Thus suppose hat $\Omega$ is unbounded. Fix $\lambda>0$ and put $u:=(\lambda+A)^{-1} v$, where $v$ belongs to $\mathcal{D}^{+}:=\{v \in \mathcal{D} ; v \geq 0\}$. Then $u \in C_{0}(\bar{\Omega})$. Suppose that $-m:=\inf u<0$. Fix $R>0$ such that $\Gamma \subset\left\{x \in \mathbb{R}^{n} ;|x|<R\right\}$ and $|u(x)| \leq m / 2$ for $|x| \geq R$. Then $u$ has a negative minimum in $\Omega_{R} \cup \Gamma$, where $\Omega_{R}:=\{x \in \Omega ;|x|<R\}$. Since $u$ satisfies $(\mathcal{A}+\lambda) u \geq 0$ in $\Omega_{R}$ and $\mathcal{B} u=0$ on $\Gamma$, this contradicts the maximum principle. Thus $u \geq 0$. Now the assertion follows from the density of $\mathcal{D}^{+}$in the positive cone of $L_{q}$.

In the rest of this section it is understood that $\left[\left(E_{\xi}, A_{\xi}\right) ;-1 \leq \xi \leq 1\right]$ is the scale defined in Lemma 3.1(ii).

We assume that

$$
s, \sigma \in I_{q}, \quad 0 \leq s<\sigma<2 .
$$

Then we set $\alpha:=s / 2$ and $\beta:=\sigma / 2$, as well as

$$
A:=A_{\alpha-1} \in \mathcal{L}\left(W_{q, \mathcal{B}}^{s}, W_{q, \mathcal{B}}^{s-2}\right) .
$$


Hence $-A$ generates a strongly continuous analytic semigroup on $W_{q, \mathcal{B}}^{s-2}$, and we consider the semilinear parabolic problem

$$
\dot{u}+A u=\mu(u) \quad \text { on } \mathbb{R}^{+},
$$

where $\mu(u) \in \mathcal{M}_{\text {loc }}\left(\mathbb{R}^{+}, W_{q, \mathcal{B}}^{\sigma-2}\right)$ has the Volterra property.

To be more precise, given Banach spaces $E$ and $F$, we write $\mu \in \mathcal{M}_{\mathrm{loc}}\left(\mathbb{R}^{+}, E\right)$ if $\mu$ is an $E$-valued measure on $\mathbb{R}^{+}$such that $\mu \mid[0, T]$ belongs to $\mathcal{M}([0, T], E)$ for every $T>0$. We also write

$$
\mu \in C_{b}^{1-}\left(L_{p, \text { loc }}\left(\mathbb{R}^{+}, F\right), \mathcal{M}_{\text {loc }}\left(\mathbb{R}^{+}, E\right)\right)
$$

to mean that

$$
\mu \mid[0, T] \in C_{b}^{1-}\left(L_{p}((0, T), F), \mathcal{M}([0, T], E)\right)
$$

for each $T>0$. Similarly, if $r \in[1, \infty]$, then

$$
f \in C_{b}^{1-}\left(L_{p, \text { loc }}\left(\mathbb{R}^{+}, F\right), L_{r, \text { loc }}\left(\mathbb{R}^{+}, E\right)\right)
$$

has the analogous meaning.

Suppose that $1 \leq p<\infty$, and that

$$
\mu \in C_{b}^{1-}\left(L_{p, \text { loc }}\left(\mathbb{R}^{+}, W_{q, \mathcal{B}}^{s}\right), \mathcal{M}_{\mathrm{loc}}\left(\mathbb{R}^{+}, W_{q, \mathcal{B}}^{\sigma-2}\right)\right)
$$

has the Volterra property.

By a (weak) $L_{p}\left(W_{q}^{s}\right)$-solution of (3.6) on $J$, where $J$ is a perfect subinterval of $\mathbb{R}^{+}$containing 0 , we mean a $u \in L_{p, \text { loc }}\left(J^{*}, W_{q, \mathcal{B}}^{s}\right)$ satisfying

$$
\int_{J}\left\langle-\partial_{t} v+\mathcal{A} v, u\right\rangle d t=\int_{J} v d \mu(u), \quad v \in \mathcal{D}\left(J^{*}, \mathcal{D}_{\mathcal{B}}\right),
$$

where $\mathcal{D}_{\mathcal{B}}:=\{v \in \mathcal{D}(\bar{\Omega}) ; \mathcal{B} v=0\}$.

Now we can prove the following basic existence, uniqueness, and continuity theorem for $L_{p}\left(W_{q}^{s}\right)$-solutions of (3.6). For an easy statement of the continuity properties we use a slightly incorrect notation. The precise meaning, however, is clear from Theorem 1.2. Often we also write $J_{T}$ for $[0, T]$.

Theorem 3.2. Suppose that $s, \sigma \in I_{q}, 0 \leq s<\sigma<2$, and

$$
1 \leq p<2 /(s-\sigma+2) \text {. }
$$

Also suppose that (3.7) is true and that $\mu$ has the Volterra property. Then problem (3.6) has a unique maximal $L_{p}\left(W_{q}^{s}\right)$-solution $u(\mu)$. The map

$$
C_{b}^{1-}\left(L_{p, \mathrm{loc}}\left(\mathbb{R}^{+}, W_{q, \mathcal{B}}^{s}\right), \mathcal{M}_{\mathrm{loc}}\left(\mathbb{R}^{+}, W_{q, \mathcal{B}}^{\sigma-2}\right)\right) \rightarrow L_{p, \mathrm{loc}}\left(\mathbb{R}^{+}, W_{q, \mathcal{B}}^{s}\right),
$$

sending $\mu$ into $u(\mu)$, is Lipschitz continuous in the sense made precise in Theorem 1.2. If $u(\mu) \in L_{p}\left(J(u(\mu)) \cap(0, T), W_{q}^{s}\right)$ for each $T>0$, then $J(u)=\mathbb{R}^{+}$.

Proof. (a) First we observe that [5, Lemma 8 and Sections 7A and 7B] guarantee that $\mathcal{D}_{\mathcal{B}}$ is dense in $W_{q^{\prime}, \mathcal{B}}^{2-s} \doteq E_{1-\alpha}^{\sharp}$. From this we infer that $\mathcal{D}\left(J^{*}, \mathcal{D}_{\mathcal{B}}\right)$ is dense in $C_{c}\left(J^{*}, E_{1-\alpha}^{\sharp}\right) \cap C^{1}\left(J^{*},\left(E_{-\alpha}^{\sharp}\right)\right.$. Furthermore, it follows from [5, Theorem 8] that

$$
\left\langle A^{\top} v, u\right\rangle=\langle\mathcal{A} v, u\rangle, \quad(v, u) \in \mathcal{D}_{\mathcal{B}} \times W_{q, \mathcal{B}}^{s} .
$$

From this we deduce that (3.8) is equivalent to (1.2).

(b) If $2 \xi, 2 \eta \in I_{q}$ satisfy $0<\eta-\xi<1$, then, provided $2 \eta \notin \mathbb{Z}$, it follows from [3. Theorem V.1.5.9] that

$$
E_{\eta-1} \doteq\left(E_{\xi-1}, E_{\xi}\right)_{\eta-\xi, q}
$$


(c) Suppose that $s, \sigma \notin \mathbb{N}$. Then the theorem is an easy consequence of (a), (b), Lemma 3.1, and Theorems 1.1 and 1.2

(d) Assume that $s$ or $\sigma$ belongs to $\mathbb{N}$. Fix $\bar{s}, \bar{\sigma} \in I_{q} \backslash \mathbb{Z}$ satisfying $s<\bar{s}<\bar{\sigma}<\sigma$ and $p<2 /(\bar{s}-\bar{\sigma}+2)$. Then (3.7) is satisfied with $s$ and $\sigma$ replaced by $\bar{s}$ and $\bar{\sigma}$, respectively. Hence (c) guarantees the existence of a unique maximal $L_{p}\left(W_{q}^{\bar{s}}\right)$ solution $\bar{u}$ of (3.6). Clearly, $\bar{u}$ is an $L_{p}\left(W_{q}^{s}\right)$-solution of (3.6) on $J(\bar{u})$.

Suppose that $w$ is any $L_{p}\left(W_{q}^{s}\right)$-solution of (3.6) on some perfect subinterval $J$ of $\mathbb{R}^{+}$containing 0 , whose interior is denoted by $\stackrel{\circ}{J}$. Then $\mu(w) \in \mathcal{M}\left(J_{T}, E_{\beta-1}\right)$ for each $T \in \stackrel{\circ}{J}$, and $w \mid J_{T}$ is a weak $L_{p}\left(E_{\alpha}\right)$-solution of the linear problem

$$
\dot{v}+A v=\mu(w) \quad \text { on } J_{T} .
$$

Since $\alpha<\bar{\alpha}:=\bar{s} / 2<\beta=\sigma / 2$, it follows from [5, Theorem 4], by replacing $\alpha$ by $\bar{\alpha}$, that $w \mid J_{T}$ is a weak $L_{p}\left(E_{\bar{\alpha}}\right)$-solution of (3.10). This being true for every $T \in \dot{J}$, we see that $w$ is an $L_{p}\left(W_{q}^{\bar{s}}\right)$-solution of (3.6) on $J$. Thus $J \subset J(u)$ and $w=u \mid J$ by the uniqueness and maximality of $u$. This proves that (3.6) has a unique maximal $L_{p}\left(W_{q}^{s}\right)$-solution in this case also. The continuity assertion follows in an obvious manner.

Now we suppose that $1<r<\infty$ with $\sigma-2 / r \in I_{q}$, and that

$$
\left(u^{0}, f\right) \in W_{q, \mathcal{B}}^{\sigma-2 / r} \times C_{b}^{1-}\left(L_{p, \text { loc }}\left(\mathbb{R}^{+}, W_{q, \mathcal{B}}^{s}\right), L_{r, \text { loc }}\left(\mathbb{R}^{+}, W_{q, \mathcal{B}}^{\sigma-2}\right)\right)
$$

with $f$ possessing the Volterra property. Then $u$ is said to be a strong $L_{p}\left(W_{q}^{s}\right)$ solution of

$$
\dot{u}+A u=f(u), \quad 0<t<\infty, \quad u(0)=u^{0}
$$

on $J$ if $u$ is an $L_{p}\left(W_{q}^{s}\right)$-solution on $J$ of (3.6) with

$$
\mu:=f+u^{0} \otimes \delta_{0}
$$

such that

$$
u \in \mathbb{W}_{r, \text { loc }}^{1}\left(J^{*},\left(W_{q, \mathcal{B}}^{s-2}, W_{q, \mathcal{B}}^{s}\right)\right) .
$$

Observe that $u(0)$ is well-defined, thanks to $u \in C\left(J^{*}, W_{q, \mathcal{B}}^{s-2}\right)$.

The next theorem guarantees the well-posedness of problem (3.12) in the sense of strong solutions.

Theorem 3.3. Suppose that $s, \sigma \in I_{q}, \quad 0 \leq s<\sigma<2$, and

$$
1<r<2 /(\sigma-s), \quad \sigma-2 / r \in I_{q}, \quad 1 \leq p<2 /(s-\sigma+2 / r) .
$$

Also assume that (3.11) is true with $f$ possessing the Volterra property. Then (3.12) has a unique maximal strong $L_{p}\left(W_{q}^{s}\right)$-solution $u\left(u^{0}, f\right)$. The map

$$
W_{q, \mathcal{B}}^{\sigma-2 / r} \times C^{1-}\left(L_{p, \text { loc }}\left(\mathbb{R}^{+}, W_{q, \mathcal{B}}^{s}\right), L_{r, \text { loc }}\left(\mathbb{R}^{+}, W_{q, \mathcal{B}}^{\sigma-2}\right)\right) \mapsto L_{p, \text { loc }}\left(\mathbb{R}^{+}, W_{q, \mathcal{B}}^{s}\right),
$$

sending $\left(u^{0}, f\right)$ into $u\left(u^{0}, f\right)$, is Lipschitz continuous in the sense made precise in Theorem 2.2. If $u\left(u^{0}, f\right) \in L_{p}\left(J\left(u\left(u^{0}, f\right)\right) \cap(0, T), W_{q, \mathcal{B}}^{s}\right)$ for each $T>0$, then $u\left(u^{0}, f\right)$ is a global solution.

Proof. This follows easily from Theorems 2.1 and 2.2 
Lastly, we consider the case $r>2 /(\sigma-s)$. Clearly, if $0 \leq \rho<1$, then $u$ is a strong $C^{\rho}\left(W_{q}^{s}\right)$-solution of (3.12) on $J$ if it belongs to $C^{\rho}\left(J, W_{q, \mathcal{B}}^{s}\right)$ and satisfies (3.8) and (3.13).

Theorem 3.4. Suppose that $s, \sigma \in I_{q}, 0 \leq s<\sigma<2$, and

$$
2 /(\sigma-s)<r<\infty, \quad 0 \leq 2 \rho<\sigma-s-2 / r .
$$

Also suppose that

$$
\left(u^{0}, f\right) \in W_{q, \mathcal{B}}^{\sigma-2 / r} \times C_{b}^{1-}\left(C^{\rho}\left(\mathbb{R}^{+}, W_{q, \mathcal{B}}^{s}\right), L_{r, \mathrm{loc}}\left(\mathbb{R}^{+}, W_{q, \mathcal{B}}^{\sigma-2}\right)\right)
$$

and that $f$ has the Volterra property. Then (3.12) has a unique maximal strong $C^{\rho}\left(W_{q}^{s}\right)$-solution $u\left(u^{0}, f\right)$. The map $\left(u^{0}, f\right) \mapsto u\left(u^{0}, f\right)$ is Lipschitz continuous from the space occurring in (3.15) into $C^{\rho}\left(\mathbb{R}^{+}, W_{q, \mathcal{B}}^{s}\right)$ in the sense made precise in Theorem [2.3. If $u\left(u^{0}, f\right)$ is uniformly $\rho$-Hölder continuous on $J\left(u\left(u^{0}, f\right)\right) \cap(0, T)$ for every $T>0$, then it is a global solution.

Proof. This follows from Theorem 2.3

Remarks 3.5. (a) The maximal $L_{p}\left(W_{q}^{s}\right)$-solution of (3.6) is independent of those $s, \sigma$, and $p$ satisfying (3.4) and (3.9) for which (3.7) is true. If $\Omega$ is bounded, then it is independent of $q$ as well.

Proof. This follows from the arguments of part (d) of the proof of Theorem 3.2 and from [5, Theorem 4], because $W_{q_{1}, \mathcal{B}}^{s} \hookrightarrow W_{q_{0}, \mathcal{B}}^{s}$ if $q_{0}<q_{1}$ and $\Omega$ is bounded.

(b) Similarly, the maximal strong $L_{p}\left(W_{q}^{s}\right)$-solution of (3.12) is independent of those $s, \sigma, r$, and $p$ satisfying (3.14) for which (3.11) is true, and of $q$, provided $\Omega$ is bounded.

(c) Suppose that $s>1 / q$. Then $u$ is an $L_{p}\left(W_{q}^{s}\right)$-solution of (3.6) on $J$ iff $u$ belongs to $L_{p, \text { loc }}\left(J^{*}, W_{q, \mathcal{B}}^{s}\right)$ and

$$
\int_{J}\left\{-\left\langle\partial_{t} v, u\right\rangle+\mathfrak{a}(v, u)\right\} d t=\int_{J} v d \mu(u), \quad v \in \mathcal{D}\left(J^{*}, \mathcal{D}\left(\Omega \cup \Gamma_{1}\right)\right),
$$

where $\mathfrak{a}(w, z):=\langle\nabla w, \boldsymbol{a} \nabla z\rangle, w, z \in \mathcal{D}(\bar{\Omega})$, is the Dirichlet form associated with $(\mathcal{A}, \mathcal{B})$.

Proof. This is a consequence of [5, Theorem 8] and the density of $\mathcal{D}\left(\Omega \cup \Gamma_{1}\right)$ in $W_{q^{\prime}, \mathcal{B}}^{2-s}$, which follows from $2-s<1+1 / q^{\prime}$.

(d) The last assertion of Theorem 3.2 is equivalent to saying that $u:=u(\mu)$ is a global solution if

$$
\mu(u) \in \mathcal{M}\left(J(u) \cap[0, T], W_{q, \mathcal{B}}^{\sigma-2}\right) \quad \text { for every } T>0 .
$$

Similarly, the last assertion of Theorem 3.3, resp. Theorem 3.4 is equivalent to saying that $u:=u\left(u^{0}, f\right)$ is a global solution if

$$
f(u) \in L_{r}\left(J(u) \cap(0, T), W_{q, \mathcal{B}}^{\sigma-2}\right) \quad \text { for every } T>0 .
$$

Proof. This follows from Remark 2.4(a).

(e) Let the hypotheses of Theorem 3.3 be satisfied. Then the corresponding solution belongs to $C\left(J(u), W_{q, \mathcal{B}}^{\tau-2 / r}\right)$ for $\tau<\sigma$ with $\tau-2 / r \in I_{q}$.

Proof. This is implied by Remark 2.4(c). 
(f) Everything said above remains valid if we suppose $\boldsymbol{a} \in B U C^{1}\left(\Omega, \mathbb{R}^{n \times n}\right)$ only, provided we replace $\mathcal{D}_{\mathcal{B}}$ by $W_{q^{\prime}, \mathcal{B}}^{2-s}$ everywhere.

Proof. For this we refer to 2 .

\section{INTERPRETATIONS}

In order to give interpretations of problem (3.6) we turn to the study of vectormeasure-valued functions $\mu$ which possess special structures occurring frequently in applications.

To simplify the presentation we assume from now on that

$$
\text { - } n \geq 2
$$

and leave to the reader the simple modifications of the following proofs which are needed to cover the case $n=1$.

Unless explicitly stated otherwise, we suppose throughout that

$$
\text { - } 1<q<n /(n-1)
$$

to avoid tedious discussions of special cases. Consequently, $0<1-n / q^{\prime}<1 / q$ and $2-n / q^{\prime} \leq n / q$.

For later reference we collect some embedding results. For this, given a smooth submanifold $M$ of $\mathbb{R}^{n}$, we denote by $C_{0}^{1}(M)$ the closure of $\mathcal{D}(M)$ in $B C^{1}(M)$, the space of bounded continuous functions having bounded and continuous first derivatives. We also put

$$
C_{\mathcal{B}}^{1}(\bar{\Omega}):=\left\{v \in C_{0}^{1}(\bar{\Omega}) ; \mathcal{B} v=0\right\} .
$$

Note that $C_{\mathcal{B}}^{1}(\bar{\Omega})=\left\{v \in C^{1}(\bar{\Omega}) ; \mathcal{B} v=0\right\}$ if $\Omega$ is bounded.

Lemma 4.1. Suppose that $t, \sigma \in I_{q} \cap \mathbb{R}^{+}$and $\sigma<2-n / q^{\prime}$. Then

(i) $W_{q, \mathcal{B}}^{t} \stackrel{d}{\hookrightarrow} L_{\rho}$ for $0<1 / q-t / n \leq 1 / \rho$ with $1 / \rho \leq 1 / q$ if $\Omega$ is unbounded;

(ii) $L_{\rho} \hookrightarrow W_{q, \mathcal{B}}^{\sigma-2}$ for $1 \leq \rho \leq q$;

(iii) $\mathcal{M}\left(\Omega \cup \Gamma_{1}\right) \hookrightarrow W_{q, \mathcal{B}}^{\sigma-2}$;

(iv) $W_{q}^{t-1 / q}\left(\Gamma_{1}\right) \hookrightarrow L_{\tau}\left(\Gamma_{1}\right)$ for $1 \geq 1 / \tau \geq(n / q-t) /(n-1)$ with $\tau$ being finite if $t=n / q$;

(v) $L_{\rho}\left(\Gamma_{1}\right) \hookrightarrow W_{q}^{\sigma-1-1 / q}\left(\Gamma_{1}\right)$ for $1 \leq \rho \leq q$;

(vi) $\mathcal{M}\left(\Gamma_{1}\right) \hookrightarrow W_{q}^{\sigma-1-1 / q}\left(\Gamma_{1}\right)$;

(vii) if $0 \leq \sigma<1-n / q^{\prime}$, then $\left(C_{\mathcal{B}}^{1}(\bar{\Omega})\right)^{\prime} \hookrightarrow W_{q, \mathcal{B}}^{\sigma-2}, \mathcal{M}\left(\Gamma_{0}\right) \hookrightarrow W_{q}^{\sigma-1 / q}\left(\Gamma_{0}\right)$, and $C^{1}\left(\Gamma_{1}\right)^{\prime} \hookrightarrow W_{q}^{\sigma-1-1 / q}\left(\Gamma_{1}\right)$.

Proof. The assertions are easy consequences of Sobolev-type embedding theorems (cf. [2, Lemma 9]) and duality arguments, where we have to use $L_{1} \hookrightarrow \mathcal{M}$ in the case where $\rho=1$.

We also observe that the trace theorem implies

$$
\gamma_{1}:=\left(u \mapsto(\gamma u) \mid \Gamma_{1}\right) \in \mathcal{L}\left(W_{q^{\prime}, \mathcal{B}}^{2-\sigma}, W_{q^{\prime}}^{1-\sigma+1 / q}\left(\Gamma_{1}\right)\right)
$$

if $\sigma<1+1 / q$, and

$$
\partial_{\boldsymbol{\nu}, 0}:=\left(u \mapsto \partial_{\boldsymbol{\nu}} u \mid \Gamma_{0}\right) \in \mathcal{L}\left(W_{q^{\prime}, \mathcal{B}}^{2-\sigma}, W_{q^{\prime}}^{-\sigma+1 / q}\left(\Gamma_{0}\right)\right)
$$

if $\sigma<1 / q$. 
Throughout the rest of this section we suppose that

$$
\text { - } s, \sigma \in\left[0,2-n / q^{\prime}\right) \cap I_{q}, \quad s<\sigma, \quad 0 \leq p<\infty, \quad 1 \leq r<\infty .
$$

In order to avoid tedious repetitions we introduce some compact notation: If $J$ is a perfect subinterval of $\mathbb{R}^{+}$containing 0 , we set

$$
X_{p, q}^{s}(J):=\left\{\begin{array}{cl}
L_{p, \operatorname{loc}}\left(J, W_{q, \mathcal{B}}^{s}\right), & 1 \leq p<\infty, \\
C^{p}\left(J, W_{q, \mathcal{B}}^{s}\right), & 0 \leq p<1,
\end{array}\right.
$$

where, of course, $C^{0}:=C$. We also put $X_{p, q}^{s}:=X_{p, q}^{s}\left(\mathbb{R}^{+}\right)$.

Local nonlinearities. Assume that

- $(F, G) \in C_{b}^{1-}\left(X_{p, q}^{s}, L_{r, \text { loc }}\left(\mathbb{R}^{+},\left(L_{1}+L_{q}\right) \times L_{1}\left(\Gamma_{1}\right)\right)\right)$;

- $F$ and $G$ possess the Volterra property.

We postpone the derivation of sufficient conditions for the validity of this assumption to Section 15 in the appendix. It follows from (4.2) and Lemma 4.1 that

$$
\mu_{\mathrm{reg}}:=F+\gamma_{1}^{\prime} G \in C_{b}^{1-}\left(X_{p, q}^{s}, X_{r, q}^{\sigma-2}\right),
$$

and $\mu_{\text {reg }}$ has the Volterra property. Furthermore, setting

$$
\langle u, v\rangle_{\Gamma_{1}}:=\int_{\Gamma_{1}} u \cdot v d \sigma, \quad u, v \in C\left(\Gamma_{1}\right),
$$

with $d \sigma$ denoting the volume measure of $\Gamma$, it follows that

$$
\begin{aligned}
\int_{J} v d \mu_{\mathrm{reg}} & =\int_{J}\left\{\langle v, F\rangle+\left\langle\gamma_{1} v, G\right\rangle_{\Gamma_{1}}\right\} d t \\
& =\int_{\Omega \times J} v F d x d t+\int_{\Gamma_{1} \times J} v G d \sigma d t
\end{aligned}
$$

for $v \in C_{0}\left(J^{*}, C_{0}\left(\Omega \cup \Gamma_{1}\right)\right)$.

Measures. Next we assume that

- $\left(\bar{\mu}, \mu_{0}\right) \in C_{b}^{1-}\left(X_{p, q}^{s}, \mathcal{M}_{\text {loc }}\left(\mathbb{R}^{+}, \mathcal{M}\left(\Omega \cup \Gamma_{1}\right) \times \mathcal{M}\left(\Gamma_{0}\right)\right)\right)$;

- $\mu_{0}=0$ if $\sigma>1 / q$;

- $\bar{\mu}$ and $\mu_{0}$ possess the Volterra property.

Then it follows from Lemma 4.1 and (4.3) that

$$
\mu_{\mathcal{M}}=\bar{\mu}-\left(\partial_{\nu, 0}\right)^{\prime} \mu_{0} \in C_{b}^{1-}\left(X_{p, q}^{s}, \mathcal{M}_{\mathrm{loc}}\left(\mathbb{R}^{+}, W_{q, \mathcal{B}}^{\sigma-2}\right)\right),
$$

and $\mu_{\mathcal{M}}$ has the Volterra property. Furthermore,

$$
\begin{aligned}
\int_{J} v d \mu_{\mathcal{M}} & =\int_{J} v d \bar{\mu}-\int_{J} \partial_{\boldsymbol{\nu}, 0} v d \mu_{0} \\
& =\int_{\left(\Omega \cup \Gamma_{1}\right) \times J} v(x, t) \bar{\mu}(d x d t)+\int_{\Gamma_{0} \times J} \partial_{\boldsymbol{\nu}, 0} v(y, t) \mu_{0}(d y d t)
\end{aligned}
$$

for $v \in C_{0}\left(J^{*}, C_{0}^{1}\left(\Omega \cup \Gamma_{1}\right)\right)$.

Here and elsewhere, given a $\sigma$-compact metric space $X$, we identify $\mathcal{M}\left(J_{T}, X\right)$ with $\mathcal{M}\left(X \times J_{T}\right)$ for $T>0$ in the obvious way (cf. [5. Example 1(d)]. We also put

$$
\mathcal{M}_{\text {loc }}\left(X \times \mathbb{R}^{+}\right):=\mathcal{M}_{\text {loc }}\left(\mathbb{R}^{+}, \mathcal{M}(X)\right) .
$$


In general, $\bar{\mu} \in \mathcal{M}_{\text {loc }}\left(\mathbb{R}^{+}, \mathcal{M}\left(\Omega \cup \Gamma_{1}\right)\right)$ charges the boundary $\Gamma_{1}$. For a neat interpretation of an $L_{p}\left(W_{q}^{s}\right)$-solution of (3.6) it is desirable to separate this contribution of $\bar{\mu}$ from its "part" in the interior $\Omega$. The following remarks show how this can be done. As usual, we omit $\Omega$ in the notation of spaces, that is, we put $C_{0}:=C_{0}(\Omega)$ and $\mathcal{M}:=\mathcal{M}(\Omega)$.

Remarks 4.2. (a) Suppose that $\Gamma_{1} \neq \emptyset$. Clearly, $\gamma_{1} \in \mathcal{L}\left(C_{0}\left(\Omega \cup \Gamma_{1}\right), C\left(\Gamma_{1}\right)\right)$, and it is a retraction. Let $\gamma_{1}^{c} \in \mathcal{L}\left(C\left(\Gamma_{1}\right), C_{0}\left(\Omega \cup \Gamma_{1}\right)\right)$ be a coretraction. Then

$$
p_{\Omega}:=\mathrm{id}-\gamma_{1}^{\mathrm{c}} \gamma_{1} \in \mathcal{L}\left(\mathrm{C}_{0}\left(\Omega \cup \Gamma_{1}\right)\right)
$$

is a projection onto $C_{0}$ (cf. the proof of [5] Theorem 10]). It is a consequence of [5. Theorem 11] that

$$
\mathcal{M}\left(\Omega \cup \Gamma_{1}\right) \cong \mathcal{M} \times \mathcal{M}\left(\Gamma_{1}\right) .
$$

An isomorphism is given by

$$
\mathcal{M}\left(\Omega \cup \Gamma_{1}\right) \rightarrow \mathcal{M} \times \mathcal{M}\left(\Gamma_{1}\right), \quad \mu \mapsto\left(r_{\Omega} \mu,\left(\gamma_{1}^{c}\right)^{\prime} \mu\right),
$$

with $r_{\Omega} \in \mathcal{L}\left(\mathcal{M}\left(\Omega \cup \Gamma_{1}\right), \mathcal{M}\right)$ being the restriction map $\mu \mapsto \mu \mid C_{0}$. Its inverse,

$$
R \in \mathcal{L}\left(\mathcal{M} \times \mathcal{M}\left(\Gamma_{1}\right), \mathcal{M}\left(\Omega \cup \Gamma_{1}\right)\right),
$$

is given by

$$
R\left(\nu_{\Omega}, \nu_{1}\right):=p_{\Omega}^{\prime} \nu_{\Omega}+\gamma_{1}^{\prime} \nu_{1}
$$

Note that

$$
\int_{\Omega \cup \Gamma_{1}} w d R\left(\nu_{\Omega}, \nu_{1}\right)=\int_{\Omega} p_{\Omega} w d \nu_{\Omega}+\int_{\Gamma_{1}} \gamma_{1} w d \nu_{1}, \quad w \in \mathcal{D}\left(\Omega \cup \Gamma_{1}\right) .
$$

(b) Suppose that $\mu \in \mathcal{M}_{\mathrm{loc}}\left(\mathbb{R}^{+}, \mathcal{M}\right)$ and denote by $\mu^{0}$ the completion of the Borel measure

$$
\mathcal{B}_{\left(\Omega \cup \Gamma_{1}\right) \times \mathbb{R}^{+}} \rightarrow \mathbb{R}, \quad B \mapsto \mu\left(B \cap\left(\Omega \times \mathbb{R}^{+}\right)\right),
$$

where $\mathcal{B}_{\left(\Omega \cup \Gamma_{1}\right) \times \mathbb{R}^{+}}$is the Borel $\sigma$-algebra of $\left(\Omega \cup \Gamma_{1}\right) \times \mathbb{R}^{+}$. Then $\mu^{0}$ belongs to $\mathcal{M}_{\text {loc }}\left(\mathbb{R}^{+}, \mathcal{M}\left(\Omega \cup \Gamma_{1}\right)\right)$ and, given $T>0$, the map

$$
\mathcal{M}\left(J_{T}, \mathcal{M}\right) \rightarrow \mathcal{M}\left(J_{T}, \mathcal{M}\left(\Omega \cup \Gamma_{1}\right)\right), \quad \mu\left|J_{T} \mapsto \mu^{0}\right| J_{T}
$$

is an isometry. We call $\mu^{0}$ the "trivial extension of $\mu$ over $\Omega \cup \Gamma_{1}$ " and put

$$
\int_{J_{T}} \varphi d \mu:=\int_{J_{T}} \varphi d \mu^{0}, \quad \varphi \in C\left(J_{T}, C_{0}\left(\Omega \cup \Gamma_{1}\right)\right), \quad T>0 .
$$

Henceforth, we usually do not distinguish notationally between $\mu \in \mathcal{M}_{\text {loc }}\left(\mathbb{R}^{+}, \mathcal{M}\right)$ and its trivial extension over $\Omega \cup \Gamma_{1}$. In other words, $\mu \in \mathcal{M}_{\text {loc }}\left(\mathbb{R}^{+}, \mathcal{M}\right)$ is identified with an element of $\mathcal{M}_{\text {loc }}\left(\mathbb{R}^{+}, \mathcal{M}\left(\Omega \cup \Gamma_{1}\right)\right)$ by means of (4.11).

(c) In [5, Lemma 10(iii)] it is claimed that

$$
\int_{0}^{\infty} p_{\Omega} v d \mu_{\Omega}=\int_{0}^{\infty} v d \mu_{\Omega}, \quad v \in \mathcal{D}\left(\mathbb{R}^{+}, \mathcal{D}_{\mathcal{B}^{\sharp}}\right),
$$

if $\mu_{\Omega} \in \mathcal{M}_{\text {loc }}\left(\mathbb{R}^{+}, \mathcal{M}\right)$ satisfies

$$
\operatorname{dist}\left(\operatorname{supp}\left(\mu_{\Omega, \mathrm{s}}\right), \Gamma_{1} \times \mathbb{R}^{+}\right)>0,
$$

where $\mu_{\Omega, s}$ is the singular part of $\mu_{\Omega}$. However, its proof, more precisely, the proof of [5 part $\beta$ of Lemma 10(i)] on which it is based, is wrong. Nevertheless, as a consequence of (b), the main results of [5], namely Theorems 1 and 2, Remark 2 and Theorem 14, remain true, even without assumption (4.12). 
For abbreviation, we set

$$
\overrightarrow{\mathcal{M}}:=\mathcal{M} \times \mathcal{M}\left(\Gamma_{0}\right) \times \mathcal{M}\left(\Gamma_{1}\right) .
$$

Motivated by the preceding remarks, we consider the following assumption:

$$
\begin{array}{ll}
\text { - } & \vec{\mu}:=\left(\mu_{\Omega}, \mu_{0}, \mu_{1}\right) \in C_{b}^{1-}\left(X_{p, q}^{s}, \mathcal{M}_{\mathrm{loc}}\left(\mathbb{R}^{+}, \overrightarrow{\mathcal{M}}\right)\right) ; \\
\text { - } & \mu_{0}=0 \text { if } \sigma>1 / q ; \\
\text { - } \vec{\mu} \text { possesses the Volterra property. }
\end{array}
$$

Then, using the identification of Remark 4.2(b), it follows from Lemma 4.1 that

$$
\mu_{\Omega}+\gamma_{1}^{\prime} \mu_{1} \in C_{b}^{1-}\left(X_{p, q}^{s}, \mathcal{M}_{\text {loc }}\left(\mathbb{R}^{+}, W_{q, \mathcal{B}}^{\sigma-2}\right)\right)
$$

and that it has the Volterra property. Note that

$$
\begin{aligned}
\int_{J} v d\left(\mu_{\Omega}+\gamma_{1}^{\prime} \mu_{1}\right) & =\int_{J} v d \mu_{\Omega}+\int_{J} \gamma_{1} v d \mu_{1} \\
& =\int_{\Omega \times J} v \mu_{\Omega}(d x d t)+\int_{\Gamma_{1} \times J} v \mu_{1}(d y d t)
\end{aligned}
$$

for $v \in C_{0}\left(J^{*}, C\left(\Omega \cup \Gamma_{1}\right)\right)$. Thus we see that (4.13) implies

$$
\chi(\vec{\mu}):=\mu_{\Omega}-\left(\partial_{\nu, 0}\right)^{\prime} \mu_{0}+\gamma_{1}^{\prime} \mu_{1} \in C_{b}^{1-}\left(X_{p, q}^{s}, \mathcal{M}_{\mathrm{loc}}\left(\mathbb{R}^{+}, W_{q, \mathcal{B}}^{\sigma-2}\right)\right) .
$$

Singular data. Suppose that $\nu_{0} \in \mathcal{M}$ and $\vec{\nu}=\left(\nu_{1}, \ldots, \nu_{n}\right) \in \mathcal{M}(\bar{\Omega})^{n}$. Put

$$
\langle\varphi, \mu\rangle:=\int_{\Omega} \varphi d \nu_{0}-\int_{\bar{\Omega}} \nabla \varphi \cdot d \vec{\nu}=\int_{\Omega} \varphi d \nu_{0}-\sum_{j=1}^{n} \int_{\bar{\Omega}} \partial_{j} \varphi d \nu_{j}
$$

for $\varphi \in C_{\mathcal{B}}^{1}(\bar{\Omega})$. It follows from Remark 4.2 (b) that (4.16) is a continuous linear form on $C_{\mathcal{B}}^{1}(\bar{\Omega})$, which we denote by $\nu_{0}+\nabla \cdot \vec{\nu}=\nu_{0}+\operatorname{div} \vec{\nu}$. Then we mean by $\mathcal{M}^{-1}$ the set of all $\mu \in C_{\mathcal{B}}^{1}(\bar{\Omega})^{\prime}$ such that there exist $\left(\nu_{0}, \vec{\nu}\right) \in \mathcal{M} \times \mathcal{M}(\bar{\Omega})^{n}$ satisfying

$$
\mu=\nu_{0}+\nabla \cdot \vec{\nu} \text {. }
$$

It is a Banach space with respect to the norm

$$
\|\mu\|_{\mathcal{M}^{-1}}:=\inf \left(\left\|\nu_{0}\right\|_{\mathcal{M}}+\|\vec{\nu}\|_{\mathcal{M}(\bar{\Omega})^{n}}\right),
$$

where the infimum is taken with respect to all representations (4.17) of $\mu$. Moreover,

$$
\mathcal{M}^{-1} \hookrightarrow\left(C_{\mathcal{B}}^{1}(\bar{\Omega})\right)^{\prime}
$$

We also put

$$
\mathcal{M}^{-1}\left(\Gamma_{1}\right):=C^{1}\left(\Gamma_{1}\right)^{\prime}
$$

Now we set

$$
\overrightarrow{\mathcal{M}}_{\text {sing }}:=\mathcal{M}^{-1} \times \mathcal{M}\left(\Gamma_{0}\right) \times \mathcal{M}^{-1}\left(\Gamma_{1}\right) .
$$

Then we suppose that

$$
\begin{array}{ll}
\text { - } & \sigma<1-n / q^{\prime} ; \\
\text { - } & \vec{F} \in C_{b}^{1-}\left(X_{p, q}^{s}, L_{1, \text { loc }}\left(\mathbb{R}^{+},\left(L_{1}+L_{q}\right)^{n}\right)\right) ; \\
\text { - } & \vec{\mu} \in C_{b}^{1-}\left(X_{p, q}^{s}, \mathcal{M}_{\text {loc }}\left(\mathbb{R}^{+}, \overrightarrow{\mathcal{M}}_{\text {sing }}\right)\right) ; \\
\text { - } & \vec{F} \text { and } \vec{\mu} \text { possess the Volterra property. }
\end{array}
$$


Note that $L_{1}+L_{q} \hookrightarrow \mathcal{M}+W_{q, \mathcal{B}}^{0}$ and $\mathcal{M}^{-1}+W_{q, \mathcal{B}}^{-1} \hookrightarrow W_{q, \mathcal{B}}^{\sigma-2}$ together with (4.20) imply

$$
\nabla \cdot \vec{F}(v) \in L_{1, \text { loc }}\left(\mathbb{R}^{+}, \mathcal{M}^{-1}+W_{q, \mathcal{B}}^{-1}\right) \hookrightarrow L_{1, \text { loc }}\left(\mathbb{R}^{+}, W_{q, \mathcal{B}}^{\sigma-2}\right)
$$

for $v \in X_{p, q}^{s}$, where

$$
\int_{J}\langle w, \nabla \cdot \vec{F}\rangle d t:=-\int_{J}\langle\nabla w, \vec{F}\rangle d t, \quad w \in C_{0}^{1}\left(J^{*}, W_{q^{\prime}, \mathcal{B}}^{2-\sigma}\right) .
$$

Thus, setting

$$
\chi(\vec{F}, \vec{\mu}):=\nabla \cdot \vec{F}+\mu_{\Omega}-\left(\partial_{\boldsymbol{\nu}, 0}\right)^{\prime} \mu_{0}+\gamma_{1}^{\prime} \mu_{1},
$$

it follows from (4.2), 4.3), 4.18, 4.19), 4.20), and Lemma 4.1(vii) that

$$
\chi(\vec{F}, \vec{\mu}) \in C_{b}^{1-}\left(X_{p, q}^{s}, \mathcal{M}_{\mathrm{loc}}\left(\mathbb{R}^{+}, W_{q, \mathcal{B}}^{\sigma-2}\right)\right) .
$$

Remarks 4.3. (a) Clearly, $\vec{\mu} \in C_{b}^{1-}\left(X_{p, q}^{s}, L_{r, \text { loc }}\left(\mathbb{R}^{+}, \overrightarrow{\mathcal{M}}\right)\right)$ implies

$$
\chi(\vec{\mu}) \in C_{b}^{1-}\left(X_{p, q}^{s}, X_{r, q}^{\sigma-2}\right) .
$$

Similarly, it follows from $\vec{\mu} \in C_{b}^{1-}\left(X_{p, q}^{s}, L_{r, \text { loc }}\left(\mathbb{R}^{+}, \overrightarrow{\mathcal{M}}_{\text {sing }}\right)\right)$ that

$$
\chi(\vec{F}, \vec{\mu}) \in C_{b}^{1-}\left(X_{p, q}^{s}, X_{r, q}^{\sigma-2}\right) .
$$

(b) If $E$ is a product of ordered Banach spaces, then we use the natural (that is, componentwise) product order on $E$. Then $\vec{\mu} \geq 0$ implies $\chi(\vec{\mu}) \geq 0$.

Of course, if $\varphi$ is a map from a nonempty set $X$ into an ordered vector space, then $\varphi \geq 0$ means $\varphi(x) \geq 0$ for all $x \in X$.

Proof. This follows from the proof of [5, Proposition 5].

Equivalence theorems. Now we are ready to give formal interpretations of the semilinear parabolic problem (3.6). For this we assume that (4.4), (4.5), and (4.13) are satisfied. Then we consider the following problem:

$$
\begin{array}{rlrlrl}
\partial_{t} u+\mathcal{A} u & =F(u)+\mu_{\Omega}(u) & & \text { in } & & \Omega \times \mathbb{R}^{+}, \\
u & =\mu_{0}(u) & & \text { on } & \Gamma_{0} \times \mathbb{R}^{+}, \\
\partial_{\boldsymbol{\nu}} u & =G(u)+\mu_{1}(u) & & \text { on } & \Gamma_{1} \times \mathbb{R}^{+} .
\end{array}
$$

By an $X_{p, q}^{s}$-solution of (4.23) on $J$ we mean a function $u \in X_{p, q}^{s}(J)$ satisfying

$$
\begin{aligned}
\int_{J}\left\langle-\partial_{t} v+\mathcal{A} v, u\right\rangle d t= & \int_{J}\langle v, F(u)\rangle d t+\int_{J} d\left\langle v, \mu_{\Omega}(u)\right\rangle-\int_{J} \partial_{\boldsymbol{\nu}, 0} v d \mu_{0}(u) \\
& +\int_{J}\left\langle\gamma_{1} v, G(u)\right\rangle_{\Gamma_{1}} d t+\int_{J} d\left\langle\gamma_{1} v, \mu_{1}(u)\right\rangle_{\Gamma}
\end{aligned}
$$

for all $v \in \mathcal{D}\left(J^{*}, \mathcal{D}_{\mathcal{B}}\right)$. Maximal solutions are defined in the obvious manner.

Theorem 4.4. Problem (4.23) is equivalent to (3.6) with

$$
\mu:=F+\gamma_{1}^{\prime} G+\chi(\vec{\mu}) ;
$$

that is, $u$ is an $X_{p, q}^{s}$-solution of (4.23) on $J$ iff it is such a solution of (3.6) with $\mu$ defined by (4.25).

Proof. This is an immediate consequence of (3.8), (4.7), (4.10), and (4.14). 
Similarly, assuming (4.4), (4.5), (4.20), and setting $\mu_{\Gamma}:=\left(\mu_{0}, \mu_{1}\right)$, we consider the problem with singular data:

$$
\begin{aligned}
& \partial_{t} u+\mathcal{A} u=F(u)+\mu_{\Omega}(u)+\nabla \cdot \vec{F}(u) \quad \text { in } \quad \Omega \times \mathbb{R}^{+}, \\
& \mathcal{B} u=\mu_{\Gamma}(u) \quad \text { on } \Gamma \times \mathbb{R}^{+} \text {. }
\end{aligned}
$$

An $X_{p, q}^{s}$-solution of (4.26) on $J$ is a $u \in X_{p, q}^{s}(J)$ satisfying

$$
\begin{aligned}
\int_{J}\left\langle-\partial_{t} v+\mathcal{A} v, u\right\rangle d t= & \int_{J}\langle v, F(u)\rangle d t+\int_{J} d\left\langle v, \mu_{\Omega}(u)\right\rangle-\int_{J}\langle\nabla v, \vec{F}(u)\rangle d t \\
& -\int_{J} \partial_{\boldsymbol{\nu}, 0} v d \mu_{0}(u)+\int_{J} d\left\langle\gamma_{1} v, \mu_{1}(u)\right\rangle_{\Gamma_{1}}
\end{aligned}
$$

for all $v \in \mathcal{D}\left(J^{*}, \mathcal{D}_{\mathcal{B}}\right)$.

The next theorem is the analogue to Theorem 4.4 for the case of singular data.

Theorem 4.5. Problem (4.26) is equivalent to (3.6) with $\mu:=F+\chi(\vec{F}, \vec{\mu})$.

Proof. This follows from (3.8), (4.7), (4.16), and (4.21).

Remarks 4.6. (a) Note that (4.24) is formally obtained from (4.23) by "multiplying" the first equation of (4.23) by $v \in \mathcal{D}\left(J^{*}, \mathcal{D}_{\mathcal{B}}\right)$, integrating over $J$, and applying Green's formula with respect to the space variables. An analogous formal argument leads from (4.26) to 4.27).

(b) Recall that $\mu_{0}=0$, and that Remark 3.5.(c) applies if $\sigma>1 / q$.

\section{Singular DATA}

In this section we consider nonlinear parabolic problems of the form

$$
\begin{aligned}
& \partial_{t} u+\mathcal{A} u=f(x, t, u)+\nabla \cdot \vec{f}(x, t, u)+\mu_{\Omega}(u)+u^{0} \otimes \delta_{0} \quad \text { in } \quad \Omega \times \mathbb{R}^{+}, \\
& \mathcal{B} u=\mu_{\Gamma}(u) \quad \text { on } \Gamma \times \mathbb{R}^{+} \text {, }
\end{aligned}
$$

where $\vec{\mu}(u)=\left(\mu_{\Omega}(u), \mu_{\Gamma}(u)\right) \in \overrightarrow{\mathcal{M}}_{\text {sing }}$. Of course, $\nabla \cdot \vec{f}(x, t, u)$ means that one has to take the divergence of the vector field $x \mapsto \vec{f}(x, t, u(x, t))$. First we derive a general result, and then we consider some special examples and model problems.

Let $X$ be a $\sigma$-compact metric space endowed with a positive Radon measure. Then, given a Carathéodory function $\varphi: X \times \mathbb{R}^{m} \rightarrow \mathbb{R}$, we use $\varphi^{\natural}$ to denote the Nemytskii operator induced by $\varphi$, defined by $\varphi^{\natural}(u):=\varphi(\cdot, u(\cdot))$ for $u: X \rightarrow \mathbb{R}^{m}$. Recall that $\varphi^{\natural}(u)$ is measurable if this is true for $u$.

The general theorem. We suppose throughout that

- $0 \leq s<1-n / q^{\prime}, 1<\lambda<\infty$, $n<\nu \leq \infty, 1 \leq r<\infty$

- $1 / \nu_{0}:=1 / q^{\prime}+s / n$;

- $\beta_{0} \in L_{\nu_{0}}, \beta \in L_{\nu}, \kappa \in L_{\infty, \text { loc }}\left(\mathbb{R}^{+}\right)$;

- $(f, \vec{f}) \in \operatorname{Car}^{1}\left(\left(\Omega \times \mathbb{R}^{+}\right) \times \mathbb{R}, \mathbb{R} \times \mathbb{R}^{n}\right)$ with $(f, \vec{f})(\cdot, \cdot, 0)=(0,0)$;

- $\left|\partial_{3} f(x, t, \xi)\right| \leq \kappa(t)\left(1+\beta(x)|\xi|^{\lambda-1}\right)$;

- $\left|\partial_{3} \vec{f}(x, t, \xi)\right| \leq \kappa(t)\left(\beta_{0}(x)+\beta(x)|\xi|^{\lambda-1}\right)$ 
for $(x, t, \xi) \in \Omega \times \mathbb{R}^{+} \times \mathbb{R}$. We also suppose that either

- $r=1$;

- $\vec{\mu} \in C_{b}^{1-}\left(L_{\lambda, \text { loc }}\left(\mathbb{R}^{+}, W_{q}^{s}\right), \mathcal{M}_{\text {loc }}\left(\mathbb{R}^{+}, \overrightarrow{\mathcal{M}}_{\text {sing }}\right)\right)$;

- $u^{0} \in \mathcal{M}^{-1}$;

where, as usual, $\vec{\mu}=\left(\mu_{\Omega}, \mu_{0}, \mu_{1}\right)=\left(\mu_{\Omega}, \mu_{\Gamma}\right)$, or

- $1<r<2 /\left(1-n / q^{\prime}-s\right)$;

- $\vec{\mu} \in C_{b}^{1-}\left(L_{r \lambda, \text { loc }}\left(\mathbb{R}^{+}, W_{q}^{s}\right), L_{r, \text { loc }}\left(\mathbb{R}^{+}, \overrightarrow{\mathcal{M}}_{\text {sing }}\right)\right)$;

- $u^{0} \in W_{q, \mathcal{B}}^{1-n / q^{\prime}-2 / r}$

or

- $2 /\left(1-n / q^{\prime}-s\right)<r<\infty, 0 \leq 2 \rho<1-n / q^{\prime}-s-2 / r$;

- $\vec{\mu} \in C_{b}^{1-}\left(C^{\rho}\left(\mathbb{R}^{+}, W_{q}^{s}\right), L_{r, \text { loc }}\left(\mathbb{R}^{+}, \overrightarrow{\mathcal{M}}_{\text {sing }}\right)\right)$;

- $u^{0} \in W_{q, \mathcal{B}}^{1-n / q^{\prime}-2 / r}$.

In each case we assume that

- $\vec{\mu}$ has the Volterra property.

We set

$$
\lambda^{*}:=\lambda^{*}(n, r, \nu):=\frac{2+n r / \nu^{\prime}}{2+(n-1) r}=1+\frac{1-n / \nu}{n-1+2 / r}
$$

and observe that $\lambda^{*}$ is increasing in $r$ and $\nu$.

Now we can prove the following general existence and uniqueness result for (5.1).

Theorem 5.1. Let assumption (5.2) be satisfied and suppose that

$$
0 \leq \frac{r}{2+(n-1) r}\left[\frac{n}{\nu^{\prime}}-\lambda\left(\frac{n}{q}-s\right)\right]<\lambda^{*}-\lambda .
$$

If $\Omega$ is unbounded, also assume that

$$
\frac{\lambda}{q} \geq \frac{s}{n}+\frac{1}{\nu^{\prime}}
$$

Moreover, let one of conditions (5.3)-(5.5), as well as assumption (5.6) be satisfied. Then problem (5.1) has a unique maximal $L_{r \lambda}\left(W_{q}^{s}\right)$-solution, $u\left(\cdot, u^{0}, \vec{\mu}\right)$, if either (5.3) or (5.4) is true, and a unique maximal $C^{\rho}\left(W_{q}^{s}\right)$-solution if (5.5) holds. In either case the solution depends Lipschitz continuously on the data.

Proof. First we note that the second inequality of (5.7) is equivalent to

$$
r \lambda\left(s-1+n / q^{\prime}+2 / r\right)<2 .
$$

From this we infer that we can fix $\sigma \in\left(s, 1-n / q^{\prime}\right)$ if either (5.3) or (5.4) is satisfied, and $\sigma \in\left(s+2 / r+2 \rho, 1-n / q^{\prime}\right)$ otherwise, with $r \lambda<2 /(s-\sigma+2 / r)$.

Set $m_{0}(x, t):=\kappa(t)$, respectively $m_{0}(x, t):=\kappa(t) \beta_{0}(x)$, and $m_{1}(x, t):=\kappa(t) \beta(x)$ for $(x, t) \in \Omega \times \mathbb{R}^{+}$. Then it follows from (5.2) and (5.8) that the hypotheses of Proposition 15.4 are satisfied. Hence

$$
(F, \vec{F}):=\left(f^{\natural}, \vec{f}^{\natural}\right) \in C_{b}^{1-}\left(X_{p, q}^{s}, L_{r, \text { loc }}\left(\mathbb{R}^{+},\left(L_{1}+L_{q}\right) \times\left(L_{1}+L_{q}\right)^{n}\right)\right),
$$


where $p:=r \lambda$ if either (5.3) or (5.4) holds, and $p:=\rho$ otherwise. From this we infer that conditions (4.5) (with $G:=0$ ) and (4.20) hold. Thus we see from (4.6) and (4.22) that

$$
\mu:=F+\chi(\vec{F}, \vec{\mu})+u^{0} \otimes \delta_{0} \in C_{b}^{1-}\left(X_{p, q}^{s}, \mathcal{M}_{\mathrm{loc}}\left(\mathbb{R}^{+}, W_{q, \mathcal{B}}^{\sigma-2}\right)\right)
$$

if $r=1$, and $\mu \in C_{b}^{1-}\left(X_{p, q}^{s}, X_{r, q}^{\sigma-2}\right)$ otherwise, thanks to Remark 4.3(a). Now Theorems 3.23 .4 imply the assertions.

Remarks 5.2. (a) Suppose that $q \in(1, n /(n-1)), \quad \lambda \in\left(1, \lambda^{*}\right), \quad \nu \in(n, \infty]$, and $r \in[1, \infty)$ with $q \leq \lambda \nu^{\prime}$ are given. Then we can find $s$ in the interval $\left[0,1-n / q^{\prime}\right)$ with $\lambda(n / q-s)=n / \nu^{\prime}$. Hence (5.7) and (5.8) are satisfied for this choice of $s$.

Proof. From $\nu>n$ and $1<\lambda<\lambda^{*}$ we deduce that

$$
1 / n^{\prime}<1 / \lambda^{*} \nu^{\prime}<1 / \lambda \nu^{\prime} \leq 1 / q \text {. }
$$

Since

$$
\left\{1 / q-s / n ; 0 \leq s<1-n / q^{\prime}\right\}=\left(1 / n^{\prime}, 1 / q\right],
$$

the assertion follows.

(b) Of course, Theorem 5.1 remains valid if we add to the right-hand side of the first equation in (5.1) a nonlocal term of the form $F_{0}(u)$, where

$$
F_{0} \in \begin{cases}C_{b}^{1-}\left(L_{r \lambda, \text { loc }}\left(\mathbb{R}^{+}, W_{q}^{s}\right), L_{r, \text { loc }}\left(\mathbb{R}^{+}, L_{1}\right)\right), & r<2 /\left(1-n / q^{\prime}-s\right), \\ C_{b}^{1-}\left(C^{\rho}\left(\mathbb{R}^{+}, W_{q}^{s}\right), L_{r, \text { loc }}\left(\mathbb{R}^{+}, L_{1}\right)\right), & \text { otherwise },\end{cases}
$$

and $F_{0}$ has the Volterra property.

(c) The assumption that $(f, \vec{f})(\cdot, \cdot, 0)=(0,0)$ is not restrictive, since a nonzero term can be included in $\mu_{\Omega}$.

(d) If assumption (5.4) holds, then the solution of Theorem 5.1 belongs to $C\left(J(u), W_{q, \mathcal{B}}^{s-2 / r}\right)$ whenever $s-2 / r \in I_{q}$ with $s<1-n / q^{\prime}$.

Proof. This follows from Remark 3.5)(e).

(e) The assumption in (5.4) and (5.5) that $u^{0} \in W_{q, \mathcal{B}}^{1-n / q^{\prime}-2 / r}$ has been imposed for simplicity. It can be replaced by

$$
u^{0} \in W_{q, \mathcal{B}}^{\sigma-2 / r},
$$

where $\sigma-2 / r \in I_{q}$ and $s<\sigma<1-n / q^{\prime}$ if either (5.3) or (5.4) is satisfied, and $s+2 / r+2 \rho<\sigma<1-n / q^{\prime}$ otherwise, provided $r \lambda<2 /(s-\sigma+2 / r)$.

Proof. This follows from the proof of Theorem 5.1.

Comparison theorems. Next we prove a comparison theorem. For this we rely on a somewhat technical version of a weak maximum principle which is relegated to the appendix.

We consider the following hypothesis:

- Assumptions (5.2), (5.7), and (5.8) are satisfied;

- if $1 \leq r<2 /\left(1-n / q^{\prime}-s\right)$, then $p:=r \lambda$ and $s+2 / r \lambda^{\prime}<\sigma<1-n / q^{\prime}$

- if $r>2 /\left(1-n / q^{\prime}-s\right)$, then $2 p \in\left[0,1-n / q^{\prime}-s-2 / r\right)$ and $s+2 / r+2 p<\sigma<1-n / q^{\prime}$. 
Theorem 5.3. Let assumption (5.10) be satisfied. Suppose that $u, v \in X_{p, q}^{s}(J)$ and there exists

$$
\left(u^{*}, \vec{\mu}^{*}\right) \in\left\{\begin{array}{rr}
\{0\} \times \mathcal{M}\left(J, \overrightarrow{\mathcal{M}}_{\text {sing }}\right), & r=1, \\
W_{q, \mathcal{B}}^{\sigma-2 / r} \times L_{r}\left(J, \overrightarrow{\mathcal{M}}_{\text {sing }}\right), & r>1,
\end{array}\right.
$$

such that

$$
\begin{array}{rlrl}
\partial_{t}(u-v)+\mathcal{A} & (u-v) & \\
& =F(u)-F(v)+\nabla \cdot(\vec{F}(u)-\vec{F}(v))+\mu_{\Omega}^{*}+u^{*} \otimes \delta_{0} & & \text { in } \Omega \times J, \\
\mathcal{B}(u-v) & =\mu_{\Gamma}^{*} & & \text { on } \Gamma \times J .
\end{array}
$$

Then $\left(u^{*}, \vec{\mu}^{*}\right) \geq 0$ implies $u \geq v$.

Proof. We can assume that $J=J_{T}$ for some $T>0$. Set

$$
\vec{c}:=-\int_{0}^{1}\left(\partial_{3} \vec{f}^{\natural}\right)(v+\tau(u-v)) d \tau, \quad a_{0}:=-\int_{0}^{1}\left(\partial_{3} f\right)^{\natural}(v+\tau(u-v)) d \tau .
$$

Put $\pi:=\pi_{0}:=r \lambda^{\prime}$ if $r<2 /\left(1-n / q^{\prime}-s\right)$, and $\pi:=\pi_{0}:=r$ otherwise. Also set $\pi_{1}:=\infty, t_{0}:=t_{1}:=s$, and $\nu_{1}:=\nu$. Then, putting $m_{0}:=\kappa$, resp. $m_{0}:=\kappa \beta_{0}$, and $m_{1}:=\kappa \beta$, we deduce from (the proof of) Lemma 15.2 that

$$
\left(\vec{c}, a_{0}\right) \in L_{\pi}\left(J_{T},\left(L_{\nu_{0}}\right)^{n} \times\left(L_{\nu_{0}}+L_{\infty}\right)\right) .
$$

Consequently, $\left(0, \vec{c}, a_{0}, 0\right) \in L_{\pi}\left(J_{T}, \mathfrak{B}\right)$ (cf. Section [19). The mean-value theorem implies that $u-v$ is a solution of the linear equation

$$
\begin{array}{rlrlrl}
\partial_{t} w-\nabla \cdot(\boldsymbol{a} \nabla w)+\nabla \cdot(\vec{c}(t) w)+a_{0}(t) w & =\mu_{\Omega}^{*}+u^{*} \otimes \delta_{0} & & \text { in } & & \Omega \times J_{T}, \\
\mathcal{B} w & =\mu_{\Gamma}^{*} & & \text { on } & \Gamma \times J_{T} .
\end{array}
$$

Thus the assertion follows from Theorem 19.4

Corollary 5.4. Let the assumptions of Theorem 5.1 be satisfied. If $\left(u^{0}, \vec{\mu}\right) \geq 0$, then $u\left(\cdot, u^{0}, \vec{\mu}\right) \geq 0$.

Proof. This follows from Theorem 5.3 with $\left(u^{*}, \vec{\mu}^{*}\right):=\left(u^{0}, \vec{\mu}(u)\right)$, where we have set $u:=u\left(\cdot, u^{0}, \vec{\mu}\right)$.

Observe that Theorem 5.3 extends, in particular, the comparison principle of Remark 2.4(e).

The next theorem is useful for proving global existence.

Theorem 5.5. Let assumption (5.10) be satisfied with $s=0$. Also suppose that one of conditions (5.3)-(5.5) as well as assumption (5.6) are true. Finally, suppose that $u_{j} \in X_{p, q}^{0}\left(J_{T}\right)$ and there exists $\left(u_{j}^{0}, \vec{\mu}_{j}\right)$ satisfying (15.11) such that

$$
\begin{aligned}
\partial_{t} u_{j}+\mathcal{A} u_{j} & =F\left(u_{j}\right)+\nabla \cdot \vec{F}\left(u_{j}\right)+\mu_{j, \Omega}+u_{j}^{0} \otimes \delta_{0} & \text { in } & \Omega \times J_{T}, \\
\mathcal{B} u_{j} & =\mu_{j, \Gamma} & \text { on } & \Gamma \times J_{T}
\end{aligned}
$$

for $j=0,1$. If $u:=u\left(\cdot, u^{0}, \vec{\mu}\right)$ and

$$
\left(u_{0}^{0}, \vec{\mu}_{0}\right) \leq\left(u^{0}, \vec{\mu}(u)\right) \leq\left(u_{1}^{0}, \vec{\mu}_{1}\right),
$$

then $J(u) \supset J_{T}$ and $u_{0} \leq u \leq u_{1}$. 
Proof. Note that $\left(u^{*}, \vec{\mu}^{*}\right):=\left(u_{1}^{0}-u^{0}, \vec{\mu}_{1}-\vec{\mu}(u)\right)$ satisfies (5.11) and is nonnegative. Furthermore,

$$
\begin{array}{ll}
\partial_{t}\left(u_{1}-u\right)+\mathcal{A}\left(u_{1}-u\right) & \\
\quad=F\left(u_{1}\right)-F(u)+\nabla \cdot\left(\vec{F}\left(u_{1}\right)-\vec{F}(u)\right)+\mu_{\Omega}^{*}+u^{*} \otimes \delta_{0} & \text { in } \Omega \times\left(J(u) \cap J_{T}\right), \\
\mathcal{B}\left(u_{1}-u\right)=\mu_{\Gamma}^{*} & \text { on } \Gamma \times\left(J(u) \cap J_{T}\right) .
\end{array}
$$

From this, the corresponding relation for $u-u_{0}$, and Theorem 5.3 we infer that

$$
u_{0} \leq u \leq u_{1} \quad \text { on } J(u) \cap J_{T} .
$$

If $1 \leq r<2 /\left(1-n / q^{\prime}-s\right)$, then this implies

$$
u \in L_{r \lambda}\left(J(u) \cap J_{T}, L_{q}\right)=X_{p, q}^{0}\left(J(u) \cap J_{T}\right) .
$$

Otherwise, (5.14) implies $u \in L_{\infty}\left(J(u) \cap J_{T}, L_{q}\right)$. From this we deduce that

$$
\mu:=F(u)+\chi(\vec{F}, \vec{\mu})(u)+u^{0} \otimes \delta_{0} \in L_{r}\left(J(u) \cap J_{T}, W_{q, \mathcal{B}}^{\sigma-2}\right) .
$$

Since $u$ satisfies the linear equation $\dot{v}+A v=\mu$, it follows from [5. Proposition 2(iii)] that

$$
u=U \star \mu \in B U C^{\rho}\left(J(u) \cap J_{T}, L_{q}\right)=X_{p, q}^{0}\left(J(u) \cap J_{T}\right) .
$$

Thus we obtain $J(u) \supset J_{T}$ from (5.15), (5.16), and Theorems 1.1] 2.1, and 2.3 respectively.

Note that conditions (5.12) and (5.13) imply

$$
\begin{aligned}
& \partial_{t} u_{0}+\mathcal{A} u_{0} \leq F\left(u_{0}\right)+\nabla \cdot \vec{F}\left(u_{0}\right)+\mu_{\Omega}(u)+u^{0} \otimes \delta_{0} \quad \text { in } \quad \Omega \times J, \\
& \mathcal{B} u_{0} \leq \mu_{\Gamma}(u) \quad \text { on } \Gamma \times J,
\end{aligned}
$$

and $u_{1}$ satisfies the corresponding system with both inequalities reversed. This means that $u_{0}$ is a subsolution and $u_{1}$ is a supersolution for problem (5.1) with $\vec{\mu}$ being "frozen" at $u\left(\cdot, u^{0}, \vec{\mu}\right)$.

In the rest of this section we consider some illuminating special cases of these general results. For simplicity, we no longer stress the fact that the solutions depend Lipschitz continuously on the data.

Examples: Local nonlinearities. First we consider the case of local nonlinearities. More precisely, we assume that $\vec{\mu}$ is independent of $u$, that is, we consider the problem

$$
\begin{aligned}
& \partial_{t} u+\mathcal{A} u=f(x, t, u)+\nabla \cdot \vec{f}(x, t, u)+\mu_{\Omega}+u^{0} \otimes \delta_{0} \quad \text { in } \quad \Omega \times \mathbb{R}^{+}, \\
& \mathcal{B} u=\mu_{\Gamma} \quad \text { on } \Gamma \times \mathbb{R}^{+} .
\end{aligned}
$$

We also strengthen assumption (5.2) by assuming that $(f, \vec{f})$ is uniformly bounded with respect to $x$. Thus we replace (5.2) by

- $(f, \vec{f}) \in \operatorname{Car}^{1}\left(\left(\Omega \times \mathbb{R}^{+}\right) \times \mathbb{R}, \mathbb{R} \times \mathbb{R}^{n}\right)$

$$
\text { with }(f, \vec{f})(\cdot, \cdot, 0)=(0,0) \text {; }
$$

- $\left|\partial_{3} f(x, t, \xi)\right| \leq \kappa(t)\left(1+|\xi|^{\lambda-1}\right)$;

- $\left|\partial_{3} \vec{f}(x, t, \xi)\right| \leq \kappa(t)|\xi|^{\lambda-1}$

for $(x, t, \xi) \in \Omega \times \mathbb{R}^{+} \times \mathbb{R}$, where $\kappa: \mathbb{R}^{+} \rightarrow \mathbb{R}^{+}$is increasing.

First we consider the case where $u^{0}=0$ and $\vec{\mu}$ is a measure with respect to $t$. 
Proposition 5.6. Let (5.18) hold with $1<\lambda<(n+2) /(n+1)$. Then problem (5.17) has for each $\left(u^{0}, \vec{\mu}\right) \in \mathcal{M}^{-1} \times \mathcal{M}_{\mathrm{loc}}\left(\mathbb{R}^{+}, \overrightarrow{\mathcal{M}}_{\text {sing }}\right)$ a unique maximal $L_{\lambda}\left(L_{\lambda}\right)$ solution. It is positive if $\left(u^{0}, \vec{\mu}\right) \geq 0$.

Proof. This is a consequence of Theorem 5.1 and Corollary 5.4 setting $\nu:=\infty$, $r:=1, s:=0$, and $q:=\lambda$.

Corollary 5.7. Suppose that

$$
\begin{aligned}
& 1 \leq \lambda_{0} \leq \lambda<(n+2) /(n+1) \\
& \text { and }\left(a_{0}, \vec{a}\right) \in L_{\infty, \text { loc }}\left(\mathbb{R}^{+}, L_{\infty} \times\left(L_{\infty}\right)^{n}\right) \text {. Then the problem } \\
& \partial_{t} u-\Delta u=a_{0} u|u|^{\lambda_{0}-1}+\nabla \cdot\left(\vec{a} u|u|^{\lambda-1}\right)+\mu_{\Omega} \quad \text { in } \quad \Omega \times \mathbb{R}^{+}, \\
& u=\mu_{0} \quad \text { on } \Gamma_{0} \times \mathbb{R}^{+}, \\
& \partial_{\nu} u=\mu_{1} \quad \text { on } \quad \Gamma_{1} \times \mathbb{R}^{+}
\end{aligned}
$$

has for each $\vec{\mu} \in \mathcal{M}_{\text {loc }}\left(\mathbb{R}^{+}, \overrightarrow{\mathcal{M}}_{\text {sing }}\right)$ a unique maximal $L_{\lambda}\left(L_{\lambda}\right)$-solution. It is positive if $\left(\mu_{\Omega}, \mu_{0}, \mu_{1}\right) \geq 0$.

Remark 5.8. In the special case of (5.17) where $\vec{\mu} \in \mathcal{M}_{\text {loc }}\left(\mathbb{R}^{+}, \overrightarrow{\mathcal{M}}\right)$ and $\vec{f}$ vanishes identically, i.e., $\vec{\mu}$ is a measure in $x$ and $t$, the restriction $\lambda<(n+2) /(n+1)$ (like the corresponding condition [6, (1.4)] for $r_{0}$ ) does not seem to be optimal. Formal computations show that $\lambda<(n+3) /(n+1)$ should be the optimal growth restriction.

Next we consider the case where $\vec{\mu} \in L_{\infty, \text { loc }}\left(\mathbb{R}^{+}, \overrightarrow{\mathcal{M}}_{\text {sing }}\right)$.

Proposition 5.9. Let (5.18) hold with $1<\lambda<n /(n-1)$. Then problem (5.17) has for each

$$
\left(\mu^{0}, \vec{\mu}\right) \in W_{\lambda}^{1-n / \lambda^{\prime}} \times L_{\infty, \text { loc }}\left(\mathbb{R}^{+}, \overrightarrow{\mathcal{M}}_{\text {sing }}\right)
$$

a unique maximal $C\left(L_{\lambda}\right)$-solution. It is positive if $\left(\mu_{0}, \vec{\mu}\right) \geq 0$.

Proof. Fix $r \in(1, \infty)$ such that $\lambda<\lambda^{*}(n, r, \infty)$ and $r>2 /\left(1-n / \lambda^{\prime}\right)$. Then assumption (5.5) is satisfied with $s=0$ and $\rho=0$. Hence the assertion follows from Theorem 5.1 and Corollary 5.4 .

The only papers known to us involving data more singular than measures are [13], 14], 24], and [25]. In all those papers $\Omega=\mathbb{R}^{n}$ and $\mu_{\Omega}=u^{0} \otimes \delta_{0}$. Furthermore, $u^{0} \in H_{q}^{-\sigma}$ for some $\sigma \in(0,2)$. In particular, in 25, Remark 5] it is observed that problem (5.19) with $\Omega=\mathbb{R}$ (that is, $n=1$ ) and $a_{0}=0$ is solvable, provided $\lambda<3 / 2$.

Examples: Transmission problems. Now we consider cases where $\mu_{\Omega}$ is a $u$-dependent singular distribution in $\mathcal{M}^{-1}$. First we study problems in which $\mu_{\Omega}(u)$ is supported on a hypersurface of $\Omega \times \mathbb{R}^{+}$.

Suppose that $S$ is an oriented $C^{1}$-hypersurface of $\bar{\Omega} \times \mathbb{R}^{+}$. Denote the positive unit normal vector field of $S$ by $\vec{N}=\left(N^{1}, \ldots, N^{n+1}\right)$, and assume that

$$
\vec{n}(x, t):=\left(N^{1}(x, t), \ldots, N^{n}(x, t)\right) \neq 0, \quad(x, t) \in S .
$$

There exists a neighborhood $U$ of $S$ in $\Omega \times \mathbb{R}^{+}$such that $U \backslash S$ consists of precisely two components $U_{+}$and $U_{-}$such that $M_{+}:=U_{+} \cup S$ and $M_{-}:=U_{-} \cup S$ are $(n+1)$-dimensional $C^{1}$-submanifolds of $\Omega \times \mathbb{R}^{+}$with common boundary $S$, that is, 
$\partial M_{+}=S=\partial M_{-}$, and such that $N$, resp. $-N$, is the outward pointing unit normal field of $M_{+}$, resp. $M_{-}$, on $S$.

If $u \in C^{1}(M)$ with $M \in\left\{M_{+}, M_{-}\right\}$, then we define the conormal derivative of $u$ at $S$ with respect to $\boldsymbol{a}$ by

$$
\partial_{\boldsymbol{n}} u:=\vec{n} \cdot \gamma_{S}(\boldsymbol{a} \nabla u)
$$

where $\gamma_{S}$ is the trace map for $S$.

We say that $u \in L_{1, \text { loc }}\left(\Omega \times \mathbb{R}^{+}\right)$is piecewise $C^{1}$ along $S$ if there exist a neighborhood $U$ of $S$ as above and $u_{ \pm} \in C^{1}\left(M_{ \pm}\right)$such that $u\left|U_{ \pm}=u_{ \pm}\right| U_{ \pm}$. If this is the case, then the jump $[u]_{S}$ of $u$, resp. $\left[\partial_{\boldsymbol{n}} u\right]_{S}$ of $\partial_{\boldsymbol{n}} u$, along $S$ is well-defined by

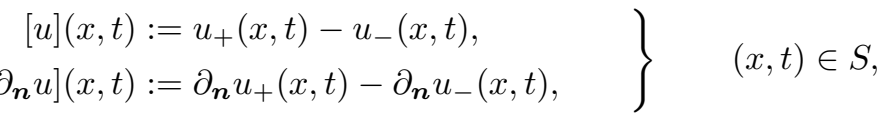

respectively, independently of the particular choice of $U$ and $u_{ \pm}$.

Lemma 5.10. Suppose that $u \in L_{1, \text { loc }}\left(\Omega \times \mathbb{R}^{+}\right)$is piecewise $C^{1}$ along $S$ and

$$
\partial_{t} u, \partial_{x}^{\alpha} u \in L_{1, \operatorname{loc}}\left(\left(\left(\Omega \times \mathbb{R}^{+}\right) \backslash S\right) \cap K\right), \quad|\alpha| \leq 2,
$$

for each compact subset $K$ of $\Omega \times \mathbb{R}^{+}$. Then, given $\varphi \in \mathcal{D}(U)$,

$$
\begin{aligned}
\int_{J}\left\langle\varphi, \partial_{t} u+\mathcal{A} u\right\rangle d t= & \int_{J}\left\langle-\partial_{t} \varphi+\mathcal{A} \varphi, u\right\rangle d t \\
& +\int_{S}[u] \partial_{\boldsymbol{n}} \varphi d \operatorname{vol}_{S}-\int_{S}\left\{\left[\partial_{\boldsymbol{n}} u\right]_{S}-N^{n+1}[u]_{S}\right\} \varphi d \operatorname{vol}_{S} .
\end{aligned}
$$

Proof. Since $S$ is a null set for the $(n+1)$-dimensional Lebesgue measure, it follows from Fubini's theorem that

$$
\begin{aligned}
\int_{J}\left\langle\varphi, \partial_{t} u+\mathcal{A} u\right\rangle d t & =\int_{U} \varphi\left(\partial_{t} u+\mathcal{A} u\right) d x d t \\
& =\left(\int_{M_{+}}+\int_{M_{-}}\right) \varphi\left(\partial_{t} u+\mathcal{A} u\right) d x d t .
\end{aligned}
$$

Thus, given $M \in\left\{M_{+}, M_{-}\right\}$,

$$
\begin{aligned}
\int_{M} \varphi\left(\partial_{t} u+\mathcal{A} u\right) d x d t= & \int_{M}\left(-\partial_{t} \varphi+\mathcal{A} \varphi\right) u d x d t \\
& +\int_{M}\left\{\partial_{t}(\varphi u)+\nabla \cdot(u \boldsymbol{a} \nabla \varphi-\varphi \boldsymbol{a} \nabla u)\right\} d x d t
\end{aligned}
$$

The divergence theorem implies

$$
\begin{aligned}
& \int_{M_{ \pm}}\left\{\partial_{t}(\varphi u)+\nabla \cdot(u \boldsymbol{a} \nabla \varphi-\varphi \boldsymbol{a} \nabla u)\right\} d x d t \\
&= \pm \int_{S}\left\{\varphi\left(N^{n+1} u_{ \pm}-\partial_{\boldsymbol{n}} u_{ \pm}\right)+u_{ \pm} \partial_{\boldsymbol{n}} \varphi\right\} d \operatorname{vol}_{S} .
\end{aligned}
$$

Hence the assertion is obvious.

For $\alpha \in L_{1, \text { loc }}(S)$ we define the "surface distribution" $\alpha \delta_{S}$ and the "dipol distribution" $\partial_{\boldsymbol{n}}\left(\alpha \delta_{S}\right)$ by

$$
\left(\alpha \delta_{S}\right)(\varphi):=\int_{S} \alpha \varphi d \operatorname{vol}_{S}, \quad \partial_{\boldsymbol{n}}\left(\alpha \delta_{S}\right)(\varphi):=-\alpha \delta_{S}\left(\partial_{n} \varphi\right), \quad \varphi \in \mathcal{D}\left(\bar{\Omega} \times \mathbb{R}^{+}\right),
$$

respectively. Then $\alpha \delta_{S} \in \mathcal{M}_{\text {loc }}\left(\Omega \times \mathbb{R}^{+}\right)$and $\partial_{\boldsymbol{n}}\left(\alpha \delta_{S}\right) \in \mathcal{M}^{-1}$. 
Now we consider the parabolic problem with transmission conditions along $S$

$$
\begin{aligned}
& \partial_{t} u+\mathcal{A} u=0 \quad \text { in } \Omega \times \mathbb{R}^{+}, \\
& \left.\begin{array}{rl}
{[u]_{S}} & =\alpha_{0} \\
{\left[\partial_{\boldsymbol{n}} u\right]_{S}} & =\alpha_{1}
\end{array}\right\} \quad \text { on } S, \\
& \mathcal{B} u=0 \quad \text { on } \Gamma \times \mathbb{R}^{+},
\end{aligned}
$$

where $\alpha_{0}, \alpha_{1} \in L_{1, \text { loc }}(S)$. By an $L_{p}\left(W_{q}^{s}\right)$-solution of (5.22) on $J$ we mean a function $u \in L_{p, \text { loc }}\left(J, W_{q}^{s}\right)$ satisfying

$$
\int_{J}\left\langle-\partial_{t} \varphi+\mathcal{A} \varphi, u\right\rangle d t=\int_{S}\left\{-\alpha_{0} \partial_{\boldsymbol{n}} \varphi+\varphi\left(\alpha_{1}-N^{n+1} \alpha_{0}\right)\right\} d \operatorname{vol}_{S}
$$

for all $\varphi \in \mathcal{D}\left(J^{*}, C_{\mathcal{B}}^{1}(\bar{\Omega})\right)$. In other words, $u \in L_{p, \text { loc }}\left(J, W_{q}^{s}\right)$ is an $L_{p}\left(W_{q}^{s}\right)$-solution of (5.22) on $J$ iff $u$ is such a solution of

$$
\dot{u}+A u=\partial_{\boldsymbol{n}}\left(\alpha_{0} \delta_{S}\right)+\left(\alpha_{1}-N^{n+1} \alpha_{0}\right) \delta_{S} .
$$

The following remark justifies this definition.

Remark 5.11. Suppose that $u \in L_{1, \text { loc }}(\Omega \times J)$, that it is piecewise $C^{1}$ along $S$, that (5.21) holds, and that it satisfies (5.22) on $(\Omega \times J) \backslash S$. Then $u$ is an $L_{p}\left(L_{q}\right)$-solution of (5.22) on $J$.

Proof. Lemma 5.10 implies the validity of (5.23) for each $\varphi \in \mathcal{D}(U)$. Now the assertion is clear.

Observe that $S$ can be interpreted as a family $\left\{S(t) ; t \in \mathbb{R}^{+}\right\}$of time-dependent interfaces in $\Omega$ along which a solution of $\partial_{t} u+\mathcal{A} u=\mu_{\Omega}(u)$ has prescribed jumps.

Let $\Sigma$ be a metric space. Then $\left\{S_{\sigma} ; \sigma \in \Sigma\right\}$ is said to be a time-like $C^{1-}$-family of oriented hypersurfaces in $\Omega \times \mathbb{R}^{+}$, provided

- $S_{\sigma}$ is for each $\sigma \in \Sigma$ an oriented $C^{1}$-hypersurface in $\Omega \times \mathbb{R}^{+}$;

- for each $\bar{\sigma} \in \Sigma$ and $(\bar{x}, \bar{t}) \in S_{\bar{\sigma}}$ there exist neighborhood

$$
V \times W \text { of }((\bar{x}, \bar{t}), \bar{\sigma}) \text { in }\left(\Omega \times \mathbb{R}^{+}\right) \times \Sigma \text { and a map }
$$

$$
\left(\sigma \mapsto \Phi_{\sigma}\right) \in C_{b}^{1-}\left(W, C^{1}(V, \mathbb{R})\right)
$$

such that $S_{\sigma} \cap V=\Phi_{\sigma}^{-1}(0)$ for $\sigma \in W$,

and $\operatorname{grad}_{x} \Phi_{\sigma}(x, t) \neq 0$ for $(x, t) \in V$ and $\sigma \in W$.

Then $\vec{N}_{\sigma}$, the positive normal of $S_{\sigma}$, has the representation

$$
\vec{N}_{\sigma}=\frac{\left(\operatorname{grad}_{x} \Phi_{\sigma}, \partial_{t} \Phi_{\sigma}\right)}{\sqrt{\left|\operatorname{grad}_{x} \Phi_{\sigma}\right|^{2}+\left(\partial_{t} \Phi_{\sigma}\right)^{2}}}
$$

since we can replace $\Phi$ by $-\Phi$, if necessary. Consequently,

$$
\vec{n}_{\sigma}(x, t)=\left(N_{\sigma}^{1}(x, t), \ldots, N_{\sigma}^{n}(x, t)\right) \neq 0, \quad(x, t) \in S_{\sigma}, \quad \sigma \in \Sigma .
$$

It follows that there exists a $C^{1}$-atlas $\mathrm{K}_{\sigma}$ for $S_{\sigma}$ such that $\kappa_{\sigma}\left(\operatorname{dom} \kappa_{\sigma}\right)=B$ for $\kappa_{\sigma} \in \mathrm{K}_{\sigma}$, where $B$ is the open unit ball in $\mathbb{R}^{n}$ if $\operatorname{dom}\left(\kappa_{\sigma}\right)$ does not intersect $\Omega \times\{0\}$, and $B:=\left\{y \in \mathbb{R}^{n} ;|y|<1, y^{n} \geq 0\right\}$ otherwise. Henceforth, we use the notation

$$
\left(\sigma \mapsto \alpha_{\sigma}\right) \in C_{b}^{1-}\left(\Sigma, L_{1, \mathrm{loc}}\left(S_{\sigma}\right)\right)
$$

to mean that

$$
\left(\sigma \mapsto\left(\alpha_{\sigma} \sqrt{g_{\sigma}}\right) \circ \kappa_{\sigma}^{-1}\right) \in C_{b}^{1-}\left(\Sigma, L_{1, \text { loc }}(B)\right), \quad \kappa_{\sigma} \in \mathrm{K}_{\sigma},
$$


where $g_{\sigma}$ is the Gram determinant of the first fundamental tensor for the standard Riemannian metric of $S_{\sigma}$ induced by the Euclidean metric of $\Omega \times \mathbb{R}$. Using these facts, it is not difficult to verify that

$$
\left(\sigma \mapsto \alpha_{j, \sigma}\right) \in C_{b}^{1-}\left(\Sigma, L_{1, \operatorname{loc}}\left(S_{\sigma}\right)\right), \quad j=0,1,
$$

implies

$$
\sigma \mapsto \partial_{\boldsymbol{n}_{\sigma}}\left(\alpha_{0, \sigma} \delta_{S_{\sigma}}\right)+\left(\alpha_{1, \sigma}-N_{\sigma}^{n+1} \alpha_{0, \sigma}\right) \delta_{S_{\sigma}} \in C_{b}^{1-}\left(\Sigma, \mathcal{M}^{-1}\right) .
$$

Now we suppose that

- $\left\{S_{\sigma} ; \sigma \in \Sigma\right\}$ is a time-like $C^{1-}$-family of oriented $C^{1-}$-hypersurfaces in $\Omega \times \mathbb{R}^{+}$.

We also suppose that

$$
\begin{array}{ll}
\text { - } & 1<\lambda<n /(n-1) \\
\text { - } & z \in C^{1-}\left(L_{\lambda}, \Sigma\right) \\
\text { - } & \left((v, \sigma) \mapsto \alpha_{j, \sigma}(v)\right) \in C^{1-}\left(L_{\lambda} \times \Sigma, L_{1, \mathrm{loc}}\left(S_{\sigma}\right)\right), j=0,1 .
\end{array}
$$

Then we put

$$
\alpha_{j}(u):=\alpha_{j, z(u)}(u), \quad j=0,1,
$$

as well as

$$
S(u):=S_{z(u)}, \quad \vec{N}(u):=\vec{N}_{z(u)}, \quad \vec{n}(u):=\vec{n}_{z(u)}
$$

for $u \in L_{\lambda}$. Then we consider the nonlinear parabolic transmission problem

$$
\begin{aligned}
& \partial_{t} u+\mathcal{A} u=f(x, t, u)+\nabla \cdot \vec{f}(x, t, u)+\mu_{\Omega} \quad \text { in } \Omega \times(0, \infty), \\
& \left.\begin{array}{rl}
{[u]_{S(u)}} & =\alpha_{0}(u) \\
u]_{S(u)} & =\alpha_{1}(u)
\end{array}\right\} \quad \text { on } S(u), \\
& \mathcal{B} u=\mu_{\Gamma} \quad \text { on } \Gamma \times(0, \infty), \\
& u(\cdot, 0)=u^{0} \quad \text { on } \Omega .
\end{aligned}
$$

Recall that the jumps on $S(u)$ occur orthogonally to the time direction, that is, they are evaluated in the actual time slice of $S(u)$. Of course, (5.28) is formal. Its precise meaning is derived from (5.23).

Possible choices for $z$ are given by

$$
z(u):=\varphi\left(\int_{\Omega_{0}} u d x\right) \quad \text { or } \quad z(u):=\varphi\left(\int_{\Omega_{0}}|u|^{\beta} d x\right), \quad \varphi \in C^{1-}(\mathbb{R}, \Sigma),
$$

where $\Omega_{0}$ is a nonempty bounded open subset of $\Omega$, and $1 \leq \beta \leq \lambda$, or combinations of such functions. In this case problem (5.28) can be interpreted as a nonlinear diffusion (heat, etc.) problem with sharp interior surfaces of discontinuity (cracks, etc.) which are moving in dependence on average densities (temperatures, etc.) in $\Omega_{0}$.

Proposition 5.12. Let (5.18), (5.26), and (5.27) be satisfied. Then problem (5.28) has for each $\left(u^{0}, \vec{\mu}\right)$ satisfying (5.20) a unique maximal $C\left(L_{\lambda}\right)$-solution.

Proof. Set

$$
\tau(u):=\partial_{\boldsymbol{n}(u)}\left(\alpha_{0}(u) \delta_{S(u)}\right)+\left(\alpha_{1}(u)-N^{n+1}(u) \alpha_{0}(u)\right) \delta_{S(u)} .
$$


Then it follows from (5.25)-(5.27) and the fact that locally Lipschitz continuous maps are uniformly continuous on compact sets that

$$
\tau \in C_{b}^{1-}\left(C\left(\mathbb{R}^{+}, L_{\lambda}\right), L_{\infty, \text { loc }}\left(\mathbb{R}^{+}, \overrightarrow{\mathcal{M}}_{\text {sing }}\right)\right) .
$$

It is obvious that $\tau$ has the Volterra property. Observe that (5.24) implies that $u$ is a $C\left(L_{\lambda}\right)$-solution of (5.28) iff it is such a solution of

$$
\dot{u}+A u=\tau(u)+\mu(u),
$$

where $\mu:=F+\chi(\vec{F}, \vec{\mu})+u^{0} \otimes \delta_{0}$. Now the assertion follows from Theorem 5.1 by the arguments of the proof of Proposition 5.9

To be more specific, we suppose that

- $\Phi \in C^{1}(\bar{\Omega} \times \mathbb{R}, \mathbb{R})$;

- $Q:=\left\{(y, \sigma) \in \bar{\Omega} \times \mathbb{R} ; \Phi(y, \sigma)=0, \nabla_{x} \Phi(y, \sigma) \neq 0\right\} \neq \emptyset$.

Then, putting

$$
\Sigma:=\{\sigma \in \mathbb{R} ; \text { there exists } x \in \bar{\Omega} \text { with }(x, \sigma) \in Q\}
$$

and

$$
M_{\sigma}:=\{x \in \bar{\Omega} ;(x, \sigma) \in Q\}, \quad \sigma \in \Sigma,
$$

it follows that $\left\{S_{\sigma}:=M_{\sigma} \times \mathbb{R}^{+} ; \sigma \in \Sigma\right\}$ is a time-like $C^{1-}$-family of $C^{1}$-hypersurfaces in $\bar{\Omega} \times \mathbb{R}^{+}$, oriented by $\vec{N}_{\sigma}:=\left(\vec{n}_{\sigma}, 0\right) \in \mathbb{R}^{n+1}$, where

$$
\vec{n}_{\sigma}:=\nabla_{x} \Phi(\cdot, \sigma), \quad \sigma \in \Sigma .
$$

In other words, $S_{\sigma}$ is a cylindrical hypersurface in $\bar{\Omega} \times \mathbb{R}^{+}$with cross-section $M_{\sigma}$ and axis parallel to $\mathbb{R}^{+}$. Thus the following result is an immediate consequence of Proposition 5.12

Corollary 5.13. Let assumptions (5.27) and (5.30) be satisfied. Also suppose that $a \in L_{\infty, \text { loc }}\left(\mathbb{R}^{+}, L_{\infty}\right)$ and $\left(u^{0}, \vec{\mu}\right)$ satisfy (5.20). Then the transmission problem with moving interfaces

$$
\begin{aligned}
& M(u):=\left\{y \in \Omega ; \Phi(u, z(u))=0, \nabla_{x} \Phi(u, z(u)) \neq 0\right\}, \\
& \partial_{t} u-\Delta u=a u|u|^{\lambda-1}+\mu_{\Omega} \quad \text { in } \quad \Omega \times(0, \infty) \text {, } \\
& \left.\begin{array}{l}
{[u]_{M(u)}=\alpha_{0}(u)} \\
u]_{M(u)}=\alpha_{1}(u)
\end{array}\right\} \quad \text { on } M(u) \times(0, \infty), \\
& \mathcal{B} u=\mu_{\Gamma} \quad \text { on } \quad \Gamma \times(0, \infty), \\
& u(\cdot, 0)=u^{0} \quad \text { on } \quad \Omega
\end{aligned}
$$

has a unique maximal $C\left(L_{\lambda}\right)$-solution. It is positive if $\alpha_{0}=0$ and $\alpha_{1} \geq 0$ as well as $\left(u^{0}, \vec{\mu}\right) \geq 0$.

Proof. For the last part, it suffices to observe that $\tau(u) \geq 0$ if $\alpha_{0}=0$ and $\alpha_{1} \geq 0$. Thus the positivity of the solution is a consequence of Corollary 5.4.

To give a simple example, we fix $\varphi, \psi \in C^{1}(\mathbb{R}, \mathbb{R})$, write $x=\left(x^{\prime}, y\right) \in \mathbb{R}^{n-1} \times \mathbb{R}$, and put

$$
\Phi(x, \sigma):=\left|x^{\prime}\right|^{2}+\varphi(\sigma) y^{2}-\psi(\sigma), \quad(x, \sigma) \in \Omega \times \mathbb{R} .
$$

Then

$$
M_{\sigma}=\left\{x \in \Omega ;\left|x^{\prime}\right|^{2}+\varphi(\sigma) y^{2}=\psi(\sigma), x \neq 0\right\}, \quad \sigma \in \mathbb{R}
$$


provided this set is not empty. Thus, depending on the signs of $\varphi(\sigma)$ and $\psi(\sigma)$, the hypersurface $M_{\sigma}$ in $\bar{\Omega}$ is the part of an $(n-1)$-dimensional ellipsoid, an $(n-1)$ dimensional hyperboloid, or an $(n-1)$-dimensional spherical cone without vertex, respectively, lying in $\bar{\Omega}$. Hence the transition surface $M(u)$ of Corollary 5.13 can deform continuously from a hypersurface of one of the preceding types to another one, disappear, and reappear in the course of the evolution, depending on the value of $z(u)$.

By imposing further restrictions we can prove that (15.31) is globally solvable. Henceforth, $\dot{J}:=J \backslash\{0\}$.

Theorem 5.14. Let assumption (5.27) be satisfied and suppose that $\alpha_{0, \sigma}=0$ and $0 \leq \alpha_{1, \sigma} \leq \bar{\alpha}<\infty$ for $\sigma \in \Sigma$. Also suppose that (5.30) is true and that $\operatorname{vol}\left(M_{\sigma}\right) \leq \omega$ for $\sigma \in \Sigma$ and some finite constant $\omega$. Finally, suppose that $a \in L_{\infty, \mathrm{loc}}\left(\mathbb{R}^{+}, L_{\infty}\right)$ and

$$
\left(u^{0}, \vec{\mu}\right) \in W_{\lambda}^{1-n / \lambda^{\prime}} \times L_{\infty, \text { loc }}\left(\mathbb{R}^{+}, \overrightarrow{\mathcal{M}}\right)
$$

with $a \leq 0$ and $\left(u^{0}, \vec{\mu}\right) \geq 0$. Then (5.31) has a unique global positive $C\left(L_{\lambda}\right)$ solution.

Proof. Fix $r \in(1, \infty)$ such that $\lambda<\lambda^{*}(n, r, \infty)$ and $r>2 /\left(1-n / \lambda^{\prime}\right)$. Set $s:=0$ and $\rho:=0$ and fix $\sigma \in\left(2 / r, 1-n / \lambda^{\prime}\right)$. Also put $q:=\lambda$.

Corollary 5.13 implies that (5.31) has a unique maximal positive $C\left(L_{\lambda}\right)$-solution, denoted by $u^{*}$. Assume $J^{*}:=J\left(u^{*}\right)=[0, T)$ with $T<\infty$. Put $\mu_{\Omega}^{*}:=\mu_{\Omega}+\bar{\alpha} \delta_{S\left(u^{*}\right)}$, and note that

$$
\left|\left\langle\bar{\alpha} \delta_{M\left(u^{*}(t)\right)}, \varphi\right\rangle\right| \leq \bar{\alpha} \int_{M\left(u^{*}(t)\right)}|\varphi| d \operatorname{vol}_{M\left(u^{*}(t)\right)} \leq \bar{\alpha} \omega\|\varphi\|_{\infty}
$$

for $\varphi \in C_{0}(\Omega)$ implies $\mu_{\Omega}^{*} \in L_{\infty}\left(J_{T}, \mathcal{M}\right)$. Set $\vec{\mu}^{*}:=\left(\mu_{\Omega}^{*}, \mu_{\Gamma}\right) \in L_{\infty}\left(J_{T}, \overrightarrow{\mathcal{M}}\right)$ and consider the linear problem

$$
\dot{v}+A v=\chi\left(\vec{\mu}^{*}\right), \quad 0<t<T, \quad v(0)=u^{0} .
$$

Since $\chi\left(\vec{\mu}^{*}\right) \in L_{\infty}\left(J_{T}, W_{q, \mathcal{B}}^{\sigma-2}\right)$ by [4.15], [5, Proposition 2(iii)] implies that $v$ belongs to $C\left(J_{T}, L_{\lambda}\right)$. Moreover, $v \geq 0$.

Put

$$
\nu^{*}:=\chi\left(\vec{\mu}^{*}\right)-F\left(u^{*}\right)-\chi(\vec{\mu})-\alpha_{1}\left(u^{*}\right) \delta_{S\left(u^{*}\right)}
$$

and note that $\nu^{*} \in L_{\infty, \text { loc }}\left(J^{*}, W_{\lambda, \mathcal{B}}^{\sigma-2}\right)$ and $\nu^{*} \geq 0$, thanks to $a \leq 0$ and $u^{*} \geq 0$. Also note that

$$
\left(v-u^{*}\right)^{\cdot}+A\left(v-u^{*}\right)=\nu^{*} \text { on } \dot{J}^{*}, \quad\left(v-u^{*}\right)(0)=0 .
$$

Consequently, $0 \leq u^{*} \leq v$ on $J^{*}$. From this it follows that $u^{*} \in L_{\infty}\left(J_{T}, L_{\lambda}\right)$. Thus $u^{*}$ solves the linear problem

$$
\dot{w}+A w=F\left(u^{*}\right)+\chi(\vec{\mu})+\alpha_{1}\left(u^{*}\right) \delta_{S\left(u^{*}\right)} \text { on } \dot{J}^{*}, \quad w(0)=u^{0},
$$

whose right-hand side belongs to $L_{\infty}\left(J_{T}, W_{\lambda, \mathcal{B}}^{\sigma-2}\right)$. Thus we infer, once more from 5, Proposition 2(iii)], that $u^{*} \in C\left(J_{T}, L_{\lambda}\right)$. But this contradicts the maximality of $J^{*}$. Consequently, $J\left(u^{*}\right)=\mathbb{R}^{+}$.

The assumption that $\operatorname{vol}\left(M_{\sigma}\right) \leq \omega<\infty$ for $\sigma \in \Sigma$ is satisfied, for example, if $\Omega$ is bounded and $M_{\sigma}$ is given by (5.32). 
Remarks 5.15. (a) It is clear that problem (5.28) can be generalized to include several transmission surfaces $S_{1}(u), \ldots, S_{m}(u)$, which, in particular, are allowed to intersect.

(b) The solutions guaranteed above are in fact $C^{\rho}\left(L_{\lambda}\right)$-solutions for sufficiently small $\rho$ (depending on the value of $\lambda$ ). Moreover, they depend continuously on the data.

(c) Suppose that $1<\lambda<(n+2) /(n+1)$ and replace the assumption on $z$ in (5.27) by $z \in C^{1-}\left(L_{\lambda, \text { loc }}\left(\mathbb{R}^{+}, L_{\lambda}\right), \Sigma\right)$ and $z$ has the Volterra property. Then (5.28) has for each

$$
\left(u^{0}, \vec{\mu}\right) \in \mathcal{M}^{-1} \times \mathcal{M}_{\text {loc }}\left(\mathbb{R}^{+}, \overrightarrow{\mathcal{M}}_{\text {sing }}\right)
$$

a unique maximal $L_{\lambda}\left(L_{\lambda}\right)$-solution.

Proof. This follows from Theorem 5.1 with $\nu:=\infty, r:=1, s:=0$, and $q:=\lambda$ by arguing as in the proof of Proposition 5.12 .

Typical examples for such $z$ are given by

$$
z(u):=\varphi\left(\int_{0}^{t} \int_{\Omega_{0}} u d x\right), \quad z(u):=\varphi\left(\int_{0}^{t} \int_{\Omega_{0}}|u|^{\beta} d x\right),
$$

where $\varphi \in C^{1-}(\mathbb{R}, \Sigma), \Omega_{0}$ is a nonempty bounded open subset of $\Omega$, and $1 \leq \beta \leq \lambda$.

Examples: Moving point sources. Now we consider another extreme situation where $\vec{\mu}$ is a measure supported on finitely many points moving in dependence on the solution $u$. More precisely, we consider the following problem:

$$
\begin{aligned}
& \partial_{t} u+\mathcal{A} u=f(x, t, u)+\nabla \cdot \vec{f}(x, t, u) \\
& +\sum_{i=0}^{k} \delta_{x_{i}(u)} \otimes \varphi_{i}+\mu_{\Omega} \quad \text { in } \quad \Omega \times(0, \infty), \\
& u=\mu_{0} \quad \text { on } \Gamma_{0} \times(0, \infty) \text {, } \\
& \partial_{\nu} u=\sum_{i=0}^{k} \delta_{y_{i}(u)} \otimes \psi_{i}+\mu_{1} \quad \text { on } \quad \Gamma_{1} \times(0, \infty), \\
& u(\cdot, 0)=u^{0} \quad \text { on } \Omega \text {. }
\end{aligned}
$$

In analogy to (5.27) we assume

- $1<\lambda<n /(n-1)$;

- $\left(x_{i}(\cdot), y_{i}(\cdot)\right) \in C_{b}^{1-}\left(L_{\lambda}, \Omega \times \Gamma_{1}\right)$ and $\left(\varphi_{i}, \psi_{i}\right) \in L_{\infty, \text { loc }}\left(\mathbb{R}^{+}, \mathbb{R} \times \mathbb{R}\right)$ for $0 \leq i \leq k$.

Problem (5.33) can be viewed as a diffusion (heat, etc.) problem with point sources whose locations move in relation to the solution $u$, more precisely, to the average densities of $u$ if $\left(x_{i}(\cdot), y_{i}(\cdot)\right)$ are defined analogously to (5.29).

Proposition 5.16. Let assumptions (5.18) and (5.34) be valid. Then problem (5.33) has for each $\left(u^{0}, \vec{\mu}\right)$ satisfying (5.20) a unique maximal $C\left(L_{\lambda}\right)$-solution. It is positive if $\left(u^{0}, \vec{\mu}\right) \geq 0$ and $\left(\varphi_{i}, \psi_{i}\right) \geq 0$ for $0 \leq i \leq k$. 
Proof. Fix $z \in\left\{x_{i}(\cdot), y_{i}(\cdot) ; 0 \leq i \leq k\right\}$ and put $M:=\Omega$ if $z=x_{i}$, and $M:=\Gamma_{1}$ if $z=y_{i}$. Then

$$
\begin{aligned}
\left|\delta_{z(u)}-\delta_{z(v)}\right|_{\mathcal{M}^{-1}(M)} & \leq c \sup _{\substack{w \in C_{0}^{1}(M) \\
\|w\|_{1}, \infty \leq 1}}\left|\left\langle\delta_{z(u)}-\delta_{z(v)}, w\right\rangle_{C(M)}\right| \\
& \leq c \sup _{\substack{w \in C_{0}^{1}(M) \\
\|w\|_{1, \infty} \leq 1}}|w(z(u))-w(z(v))| \leq c|z(u)-z(v)|
\end{aligned}
$$

for $u, v \in L_{\lambda}$, as follows from the mean value theorem (in local coordinates). From this and from assumption (5.34) we infer, setting

$$
\left(\widetilde{\mu}_{\Omega}, \widetilde{\mu}_{1}\right):=\left(\sum_{i=0}^{k} \delta_{x_{i}(\cdot)} \otimes \varphi_{i}, \sum_{i=0}^{k} \delta_{y_{i}(\cdot)} \otimes \psi_{i}\right) \in L_{\infty, \text { loc }}\left(\mathbb{R}^{+}, \mathcal{M} \times \mathcal{M}\left(\Gamma_{1}\right)\right),
$$

that

$$
\left(\widetilde{\mu}_{\Omega}, \widetilde{\mu}_{1}\right) \in C_{b}^{1-}\left(C\left(\mathbb{R}^{+}, L_{\lambda}\right), L_{\infty, \mathrm{loc}}\left(\mathbb{R}^{+}, \mathcal{M}^{-1} \times \mathcal{M}^{-1}\left(\Gamma_{1}\right)\right)\right) .
$$

Now the assertion follows once more from Theorem 5.1 the proof of Proposition 5.9 and Corollray 5.4

Remarks 5.17. (a) Observations analogous to Remarks 5.15(b) and (c) hold in this case also.

(b) Sometimes we can replace the nonlocal operators $\left(x_{i}, y_{i}\right), 0 \leq i \leq k$, by local ones. To see this we consider the model problem

$$
\begin{aligned}
\partial_{t} u-\Delta u & =\sum_{i=0}^{k} \delta_{x_{i}(u)} \otimes \varphi_{i} & & \text { in } \quad \Omega \times(0, \infty), \\
u & =0 & & \text { on } \Gamma \times(0, \infty), \\
u(\cdot, 0) & =u^{0} & & \text { on } \Omega,
\end{aligned}
$$

where

$$
x_{i}(u):=X_{i}\left(u\left(x_{0}^{*}, \cdot\right), \ldots, u\left(x_{m}^{*}, \cdot\right)\right), \quad 0 \leq i \leq k,
$$

and $x_{0}^{*}, \ldots, x_{m}^{*}$ are given points in $\Omega$. More precisely, let $K$ be a compact subset of $\Omega$ with $x_{j}^{*} \notin K$ and assume that $X_{i} \in C^{1-}\left(\mathbb{R}^{m+1}, K\right)$ for $0 \leq i \leq k$. Given points $y_{0}, \ldots, y_{m}, z_{0}, \ldots, z_{m} \in K$, let $v$, resp. $w$, be the solution on $[0, T]$ of $(5.35)$ with $x_{i}(u)$ replaced by $y_{i}$, resp. $z_{i}$, for $0 \leq i \leq k$. Then $v$ and $w$ are solutions of the homogeneous heat equation in $(\Omega \backslash K) \times[0, T]$. Hence they are smooth on $(\Omega \backslash K) \times[0, T]$, provided $u^{0}$ is appropriately smooth. Furthermore, standard interior regularity estimates yield

$$
\|v(t)-w(t)\|_{C^{\rho}\left(\Omega_{\varepsilon}\right)} \leq c\|v-w\|_{C\left([0, T], W_{q, \mathcal{B}}^{s}\right)},
$$

where $\Omega_{\varepsilon}$ is a compact $\varepsilon$-neighborhood of $\left\{x_{0}^{*}, \ldots, x_{m}^{*}\right\}$ in $\Omega \backslash K$. Now it is clear how to modify the proof of Remark 2.4(a) by incorporating condition (5.36) in the definition of the space in which Banach's fixed point theorem is applied in order to get the assertion. Details are left to the reader.

(c) Similarly as for (5.31), it is not difficult to give sufficient conditions for (5.33) to be globally solvable. For example, this is the case if (5.34) is satisfied, $f \leq 0$, $\vec{f}=0$, and $u^{0}, \vec{\mu}$, and the $\left(\varphi_{j}, \psi_{j}\right)$ are positive.

Proof. This follows analogously to the proof of Theorem5.14. 


\section{Measure Data}

Now we apply our general results to the case where we have homogeneous Dirichlet data and measures in $\Omega$ and on the Neumann boundary. In this case we can allow nonlinear gradient dependence. More precisely, we consider the problem

$$
\begin{array}{rlrlrl}
\partial_{t} u+\mathcal{A} u & =f(x, t, u, \nabla u)+\mu_{\Omega}(u) & & \text { in } & & \Omega \times \mathbb{R}^{+}, \\
u & =0 & & \text { on } & \Gamma_{0} \times \mathbb{R}^{+}, \\
\partial_{\boldsymbol{\nu}} u & =g(x, t, u)+\mu_{1}(u) & & \text { on } & \Gamma_{1} \times \mathbb{R}^{+} .
\end{array}
$$

The general theorem. We suppose throughout that

- $s_{j} \in\left[1,2-n / q^{\prime}\right), \lambda_{j} \in(1, \infty)$ for $0 \leq j \leq 4$;

- $1 \leq r<2 /\left(2-n / q^{\prime}-s_{j}\right)$ for $0 \leq j \leq 4$;

- $\nu_{0}, \nu_{1} \in(n / 2, \infty], \nu_{2}, \nu_{3} \in(n, \infty], \nu_{4} \in(n-1, \infty]$.

We also assume that

- $f \in \operatorname{Car}^{1}\left(\left(\Omega \times \mathbb{R}^{+}\right) \times\left(\mathbb{R} \times \mathbb{R}^{n}\right), \mathbb{R}\right)$ with $f_{0}:=f(\cdot, \cdot, 0,0) \in L_{r, \text { loc }}\left(\mathbb{R}^{+}, L_{1}+L_{q}\right)$;

- $\kappa: \mathbb{R}^{+} \rightarrow \mathbb{R}^{+}$is increasing, $\beta_{j} \in L_{\nu_{j}}$ for $0 \leq j \leq 3$;

- $\left|\partial_{3} f(x, t, \xi, \eta)\right| \leq \kappa(t)\left(1+\beta_{0}(x)|\xi|^{\lambda_{0}-1}+\beta_{1}(x)|\eta|^{\lambda_{1}-1}\right)$, $\left|\partial_{4} f(x, t, \xi, \eta)\right| \leq \kappa(t)\left(1+\beta_{2}(x)|\xi|^{\lambda_{2}-1}+\beta_{3}(x)|\eta|^{\lambda_{3}-1}\right)$ for $(x, t, \xi, \eta) \in \Omega \times \mathbb{R}^{+} \times \mathbb{R} \times \mathbb{R}^{n}$

and

- $g \in \operatorname{Car}^{1}\left(\left(\Gamma_{1} \times \mathbb{R}^{+}\right) \times \mathbb{R}, \mathbb{R}\right)$ with $g_{0}:=g(\cdot, \cdot, 0) \in L_{r, \text { loc }}\left(\mathbb{R}^{+}, L_{1}\left(\Gamma_{1}\right)\right)$;

- $\beta_{4} \in L_{\nu_{4}}\left(\Gamma_{1}\right)$ and $\left|\partial_{3} g(y, t, \xi)\right| \leq \kappa(t)\left(1+\beta_{4}(y)|\xi|^{\lambda_{4}-1}\right)$ for $(y, t, \xi) \in \Gamma_{1} \times \mathbb{R}^{+} \times \mathbb{R}$.

As for the measures $\mu_{\Omega}$ and $\mu_{1}$, we suppose, using the notations of Section 15 with $\vec{\lambda}:=\left(\lambda_{0}, \ldots, \lambda_{4}\right)$ and $\vec{s}:=\left(s_{0}, \ldots, s_{4}\right)$, that either

- $r=1$;

- $\left(\mu_{\Omega}, \mu_{1}\right) \in C_{b}^{1-}\left(X_{\vec{\lambda}, q}^{\vec{s}}, \mathcal{M}_{\text {loc }}\left(\mathbb{R}^{+}, \mathcal{M} \times \mathcal{M}\left(\Gamma_{1}\right)\right)\right)$, and it has the Volterra property

or

- $r>1,(n+1) / q+2 / r \notin\{1,2\}$;

- $\left(\mu_{\Omega}, \mu_{1}\right)=\left(h_{\Omega}+u^{0} \otimes \delta_{0}, h_{1}\right)$ with $\left(h_{\Omega}, h_{1}\right) \in C_{b}^{1-}\left(X_{r \vec{\lambda}, q}^{\vec{s}}, L_{r, \text { loc }}\left(\mathbb{R}^{+}, \mathcal{M} \times \mathcal{M}\left(\Gamma_{1}\right)\right)\right)$, and it has the Volterra property;

- $u^{0} \in W_{q, \mathcal{B}}^{2-n / q^{\prime}-2 / r}$.

We define numbers $\lambda_{j}^{*}:=\lambda_{j}^{*}\left(n, r, \nu_{j}\right)$ by

$$
\begin{array}{lll}
\lambda_{0}^{*}(2+(n-2) r)=2+r n / \nu_{0}^{\prime}, & \lambda_{1}^{*}(2+(n-1) r)=2+r n / \nu_{1}^{\prime}+r, \\
\lambda_{2}^{*}(2+(n-2) r)=2+r n / \nu_{2}^{\prime}-r, & \lambda_{3}^{*}(2+(n-1) r)=2+r n / \nu_{3}^{\prime}, \\
\lambda_{4}^{*}(2+(n-2) r)=2+r(n-1) / \nu_{4}^{\prime} . & &
\end{array}
$$


Observe that the restrictions for the $\nu_{j}$ of (6.2) guarantee that $\lambda_{j}^{*}>1$ for $0 \leq j \leq 4$.

The following theorem is the analogue to Theorem 5.1 for the present situation.

Theorem 6.1. Let assumptions (6.2)-(6.4) and either (6.5) or (6.6) be satisfied. Also suppose that

$$
\begin{aligned}
0 & \leq \frac{r}{2+(n-2) r}\left[\frac{n}{\nu_{0}^{\prime}}-\lambda_{0}\left(\frac{n}{q}-s_{0}\right)\right]<\lambda_{0}^{*}-\lambda_{0}, \\
0 & \leq \frac{r}{2+(n-1) r}\left[\frac{n}{\nu_{1}^{\prime}}+1-\lambda_{1}\left(\frac{n}{q}-s_{1}+1\right)\right]<\lambda_{1}^{*}-\lambda_{1}, \\
0 & \leq \frac{r}{2+(n-2) r}\left[\frac{n}{\nu_{2}^{\prime}}-1-\lambda_{2}\left(\frac{n}{q}-s_{2}\right)\right]<\lambda_{2}^{*}-\lambda_{2}, \\
0 & \leq \frac{r}{2+(n-1) r}\left[\frac{n}{\nu_{3}^{\prime}}-\lambda_{3}\left(\frac{n}{q}-s_{3}+1\right)\right]<\lambda_{3}^{*}-\lambda_{3}, \\
0 & \leq \frac{r}{2+(n-2) r}\left[\frac{n-1}{\nu_{4}^{\prime}}-\lambda_{4}\left(\frac{n}{q}-s_{4}\right)\right]<\lambda_{4}^{*}-\lambda_{4} .
\end{aligned}
$$

If $\Omega$ is unbounded, then assume, moreover, that

$$
\begin{aligned}
& \lambda_{0} / q \geq s_{0} / n+1 / \nu_{0}^{\prime}, \quad \lambda_{1} / q \geq s_{1} / n+1 / \nu_{1}^{\prime}, \\
& \lambda_{2} / q \geq\left(s_{2}-1\right) / n+1 / \nu_{2}^{\prime}, \quad \lambda_{3} / q \geq\left(s_{3}-1\right) / n+1 / \nu_{3}^{\prime} .
\end{aligned}
$$

Then (6.1) has a unique maximal $L_{r \vec{\lambda}}\left(W_{q}^{\vec{s}}\right)$-solution $u$, which is strong if $r>1$. It depends Lipschitz continuously on $f, \mu_{\Omega}, g$, and $\mu_{1}$.

Proof. The strict inequalities of conditions (6.7) are equivalent to

$$
r \lambda_{j}\left(s_{j}-2+n / q^{\prime}+2 / r\right)<2, \quad 0 \leq j \leq 4 .
$$

Thus we can fix $\sigma \in\left(\max s_{j}, 2-n / q^{\prime}\right)$ with $\sigma-2 / r \in I_{q}$ such that

$$
r \lambda_{j}<2 /\left(s_{j}-\sigma+2 / r\right), \quad 0 \leq j \leq 4 .
$$

From the inequalities on the left of (6.7), (6.8), and Propositions 15.6 and 15.7 we infer, thanks to 6.3 and 6.4 that

$$
(F, G) \in C_{b}^{1-}\left(X_{r \vec{\lambda}, q}^{\vec{s}}, L_{r, \text { loc }}\left(\mathbb{R}^{+},\left(L_{1}+L_{q}\right) \times L_{1}\left(\Gamma_{1}\right)\right),\right.
$$

where $F$ and $G$ possess the Volterra property, of course. Thus, setting

$$
\mu:=F+\gamma_{1}^{\prime} G+\mu_{\Omega}+\gamma_{1}^{\prime} \mu_{1}
$$

it follows from (6.5), (6.6), (4.6), 4.8), (4.9), and Remarks 4.2(b) and 4.3) (a) that

$$
\mu \in C_{b}^{1-}\left(X_{\vec{\lambda}, q}^{\vec{s}}, \mathcal{M}_{\mathrm{loc}}\left(\mathbb{R}^{+}, W_{q, \mathcal{B}}^{\sigma-2}\right)\right)
$$

if $r=1$, and

$$
\mu-u^{0} \otimes \delta_{0} \in C_{b}^{1-}\left(X_{r \vec{\lambda}, q}^{\vec{s}}, X_{r, q}^{\sigma-2}\right), \quad r>1 .
$$

Now the assertion is a consequence of Theorem 4.4, (6.9), Theorems 3.2 and 3.3 and Remark 2.4(d).

Remarks 6.2. (a) Suppose that $q \in(1, n /(n-1)), \quad \lambda_{j} \in\left(1, \lambda_{j}^{*}\right)$, and $r \in[1, \infty)$, as well as $\nu_{j} \in(1, \infty]$, satisfying the restrictions of ([6.2), are given. Put

$$
\begin{array}{rlrl}
d_{0}:=\frac{1}{\lambda_{0} \nu_{0}^{\prime}}, & d_{1}:=\frac{1}{\lambda_{1}}\left(\frac{1}{\nu_{1}^{\prime}}+\frac{1}{n}\right)-\frac{1}{n}, & d_{2}:=\frac{1}{\lambda_{2}}\left(\frac{1}{\nu_{2}^{\prime}}-\frac{1}{n}\right), \\
d_{3}:=\frac{1}{\lambda_{3} \nu_{3}^{\prime}}-\frac{1}{n}, & d_{4}:=\frac{1}{\lambda_{4} \nu_{4}^{\prime} n^{\prime}},
\end{array}
$$


and define $d_{j}^{*}$ for $j=0, \ldots, 4$ by replacing $\lambda_{j}$ in the definition of $d_{j}$ by $\lambda_{j}^{*}$. Suppose that

$$
1 / q \geq 1 / n+d_{j}, \quad 0 \leq j \leq 4 .
$$

Then there exist $s_{j} \in\left[1,2-n / q^{\prime}\right)$ for $0 \leq j \leq 4$ such that all squared brackets in (6.7) vanish. Hence (6.7) and (6.8) are satisfied for this choice of $s_{0}, \ldots, s_{4}$.

It is easy to see that $1 / n+d_{j}^{*}<1$ for $0 \leq j \leq 4$, unless

$$
j=0, \quad n=2, \quad r=1, \quad \nu_{0}=\infty .
$$

As a consequence of Remark 15.3, in all cases, except for (6.11), we can assume that $1 / n+d_{j}<1$, so that (6.10) is satisfied for $q$ sufficiently close to 1 . If (6.11) is true, then a choice of $q$ close to 1 and $s_{0}:=1$ guarantee (6.7), (6.8) as well.

Proof. First note that

$$
\left\{1 / q-s / n ; 1 \leq s<2-n / q^{\prime}\right\}=(1-2 / n, 1 / q-1 / n]
$$

and that the squared brackets in (6.7) vanish iff $1 / q-s_{j} / n=d_{j}$ for all $j$. Since it is easily verified that $d_{j}>d_{j}^{*} \geq 1-2 / n$, the assertion follows.

(b) Suppose that

$$
r>2 /\left(2-n / q^{\prime}-s_{j}\right), \quad 0 \leq 2 \rho<2-n / q^{\prime}-s_{j}-2 / r
$$

for $0 \leq j \leq 4$. Replace $X_{r \vec{\lambda}, q}^{\vec{s}}$ in (6.5) and (6.6) by $C^{\rho}\left(\mathbb{R}^{+}, \bigcap_{j=0}^{4} W_{q, \mathcal{B}}^{s_{j}}\right)$. Then problem (6.1) has a unique maximal $C^{\rho}\left(\bigcap_{j=0}^{4} W_{q}^{s_{j}}\right)$-solution, provided the hypotheses of Theorem 6.1 are satisfied.

Proof. The proof of Theorem 6.1 applies in this case also, with the modification that Theorem 3.4 is invoked.

(c) Of course, if $g=0$, then $\vec{\lambda}$ and $\vec{s}$ have to be replaced by $\vec{\lambda}:=\left(\lambda_{0}, \ldots, \lambda_{3}\right)$ and $\vec{s}:=\left(s_{0}, \ldots, s_{3}\right)$, respectively. Similarly, if $f$ is independent of $u$ (or of $\nabla u$, respectively), then $\left(\lambda_{0}, \lambda_{1}, \lambda_{2}\right)$ and $\left(s_{0}, s_{1}, s_{2}\right)$ (respectively, $\left(\lambda_{1}, \lambda_{2}, \lambda_{3}\right)$ and $\left.\left(s_{1}, s_{2}, s_{3}\right)\right)$ have to be omitted in the definition of $\vec{\lambda}$ and $\vec{s}$, respectively.

A comparison result. Problem (6.1) possesses a comparison theorem as well, analogous the one for (4.26).

Theorem 6.3. Let the assumptions of Theorem 6.1 be true and suppose that $\sigma$, belonging to $\left(\max s_{j}, 2-n / q^{\prime}\right) \cap I_{q}$, satisfies $\sigma-2 / r \in I_{q}$ and (6.9). Also assume that $u$ and $v$ belong to $L_{r \vec{\lambda}, \text { loc }}\left(J, W_{q}^{\vec{s}}\right)$ and $\mu_{0}^{*} \in \mathcal{M}_{\text {loc }}\left(J, \mathcal{M}\left(\Gamma_{0}\right)\right)$ is such that there exists a unique $w_{0} \in L_{1, \mathrm{loc}}\left(J, W_{q}^{1}\right)$ satisfying

$$
\begin{aligned}
& \partial_{t} w_{0}+\mathcal{A} w_{0}=0 \quad \text { in } \quad \Omega \times \dot{J}, \\
& \gamma w_{0}=\mu_{0}^{*} \quad \text { on } \quad \Gamma_{0} \times \dot{J}, \\
& \partial_{\nu} w_{0}=0 \quad \text { on } \quad \Gamma_{1} \times \dot{J}, \\
& w_{0}(\cdot, 0)=0 \quad \text { on } \quad \Omega \text {. }
\end{aligned}
$$

Finally, assume that there is

$$
\left(u^{*}, \vec{\mu}^{*}\right) \in\left\{\begin{aligned}
\{0\} & \times \mathcal{M}\left(J,\left(\mathcal{M}+L_{q}\right) \times\{0\} \times \mathcal{M}\left(\Gamma_{1}\right)\right), & & r=1, \\
W_{q, \mathcal{B}}^{\sigma-2 / r} & \times L_{r}\left(J,\left(\mathcal{M}+L_{q}\right) \times\{0\} \times \mathcal{M}\left(\Gamma_{1}\right)\right), & & r>1,
\end{aligned}\right.
$$


such that

$$
\begin{aligned}
& \partial_{t}(u-v)+\mathcal{A}(u-v)=F(u)-F(v)+\mu_{\Omega}^{*} \quad \text { in } \quad \Omega \times \dot{J}, \\
& \gamma(u-v)=\mu_{0}^{*} \quad \text { on } \Gamma_{0} \times \dot{J} \text {, } \\
& \partial_{\nu}(u-v)=G(u)-G(v)+\mu_{1}^{*} \quad \text { on } \quad \Gamma_{1} \times \dot{J}, \\
& (u-v)(\cdot, 0)=u^{*} \quad \text { on } \quad \Omega \text {. }
\end{aligned}
$$

Then $\left(u^{*}, \mu_{\Omega}^{*}, \mu_{0}^{*}, \mu_{1}^{*}\right) \geq 0$ implies $u \geq v$.

Proof. Note that

$$
\vec{\mu}(u, v):=(F(u)-F(v), 0, G(u)-G(v)) \in L_{r, \text { loc }}\left(J,\left(L_{1}+L_{q}\right) \times\{0\} \times L_{1}\left(\Gamma_{1}\right)\right) .
$$

Hence $\left(u^{*}, \mu(u, v)+\vec{\mu}^{*}\right)$ satisfies (6.12). Consequently, there exists a unique $L_{r}\left(W_{q}^{1}\right)$-solution $w_{1}$ of the linear problem

$$
\begin{aligned}
& \partial_{t} w+\mathcal{A} w=F(u)-F(v)+\mu_{\Omega}^{*} \quad \text { in } \quad \Omega \times \dot{J}, \\
& \gamma w=0 \quad \text { on } \Gamma_{0} \times \dot{J}, \\
& \partial_{\boldsymbol{\nu}} w=G(u)-G(v)+\mu_{1}^{*} \quad \text { on } \quad \Gamma_{1} \times \dot{J}, \\
& w(\cdot, 0)=u^{*} \quad \text { on } \Omega \text {. }
\end{aligned}
$$

Clearly, $u-v=w_{0}+w_{1}$, and $\mu_{0}^{*} \geq 0$ implies $w_{0} \geq 0$. Thus it suffices to prove the assertion if $\mu_{0}^{*}=0$. In this case an obvious modification of the proof of Theorem 5.3 implies the assertion.

Corollary 6.4. Let the hypotheses of Theorem 6.1 be satisfied. If $\left(f_{0}, g_{0}, \mu_{\Omega}, \mu_{1}\right)$ is positive, then the solution of (6.1) is positive as well.

We leave it to the reader to formulate and prove a "sub- and supersolution theorem" analogous to Theorem 5.5 .

Model problems. In order to simplify Theorem 6.1 we restrict ourselves to the case where $f$ and $g$ are uniformly bounded with respect to $x$ and $t$ (so that $\nu_{j}=\infty$ ). More precisely, we first suppose that $r=1$ and that the numbers $\lambda_{0}, \ldots, \lambda_{4}>1$ satisfy

$$
\begin{aligned}
& \lambda_{0}<1+2 / n, \quad \lambda_{1}<1+2 /(n+1), \\
& \lambda_{2}<1+1 / n, \quad \lambda_{3}<1+1 /(n+1), \quad \lambda_{4}<1+1 / n .
\end{aligned}
$$

Moreover, denoting, as usual, by $\kappa$ a positive increasing function on $\mathbb{R}^{+}$and assuming that $f$ and $g$ are $C^{1}$-Carathéodory functions, we suppose that

$$
\begin{aligned}
\left|\partial_{3} f(x, t, \xi, \eta)\right| & \leq \kappa(t)\left(1+|\xi|^{\lambda_{0}-1}+|\eta|^{\lambda_{1}-1}\right), \\
\left|\partial_{4} f(x, t, \xi, \eta)\right| & \leq \kappa(t)\left(1+|\xi|^{\lambda_{2}-1}+|\eta|^{\lambda_{3}-1}\right), \\
\mid \partial_{3} g(y, t, \xi \mid & \leq \kappa(t)\left(1+|\xi|^{\lambda_{4}-1}\right)
\end{aligned}
$$

for $x \in \Omega, y \in \Gamma_{1}, \quad t \in \mathbb{R}^{+}$, and $(\xi, \eta) \in \mathbb{R} \times \mathbb{R}^{n}$, and, for simplicity, that

$$
(f(\cdot, \cdot, 0,0), g(\cdot, \cdot, 0))=(0,0) .
$$


Proposition 6.5. Let (6.13) - 6.15) be true. Then there exist $q \in(1, n /(n-1))$ and $s_{j} \in\left[1,2-n / q^{\prime}\right)$ for $0 \leq j \leq 4$ such that the problem

$$
\begin{aligned}
& \partial_{t} u+\mathcal{A} u=f(x, t, u, \nabla u)+\mu_{\Omega} \quad \text { in } \quad \Omega \times \mathbb{R}^{+}, \\
& u=0 \quad \text { on } \Gamma_{0} \times \mathbb{R}^{+} \text {, } \\
& \partial_{\nu} u=g(x, t, u)+\mu_{1} \quad \text { on } \quad \Gamma_{1} \times \mathbb{R}^{+}
\end{aligned}
$$

has for each

$$
\left(\mu_{\Omega}, \mu_{1}\right) \in \mathcal{M}_{\mathrm{loc}}\left(\mathbb{R}^{+}, \mathcal{M} \times \mathcal{M}\left(\Gamma_{1}\right)\right)
$$

a unique maximal $L_{\vec{\lambda}}\left(W_{q}^{\vec{s}}\right)$-solution $u$, and $u \geq 0$ if $\left(\mu_{\Omega}, \mu_{1}\right) \geq 0$.

Proof. This follows from Theorem 6.1 Remark 6.2(a), and Corollary6.4.

Setting $\mu_{\Omega}:=u^{0} \otimes \delta_{0}$ with $u^{0} \in \mathcal{M}$, and $\mu_{1}:=0$, Proposition 6.5 guarantees the unique solvability of

$$
\begin{aligned}
& \partial_{t} u-\Delta u=f(x, t, u, \nabla u) \quad \text { in } \quad \Omega \times(0, \infty), \\
& u=0 \quad \text { on } \Gamma_{0} \times(0, \infty) \text {, } \\
& \partial_{\nu} u=g(x, t, u) \quad \text { on } \quad \Gamma_{1} \times(0, \infty) \text {, } \\
& u(\cdot, 0)=u^{0} \quad \text { on } \Omega .
\end{aligned}
$$

This particular case, and subcases thereof like

$$
\begin{aligned}
& \partial_{t} u-\Delta u=u|u|^{\lambda-1} \quad \text { in } \quad \Omega \times(0, \infty), \\
& u=0 \quad \text { on } \Gamma \times(0, \infty), \\
& u(\cdot, 0)=u^{0} \quad \text { on } \Omega,
\end{aligned}
$$

were studied by many authors (see [18, 10], 11], [12, 7], 8], [26], for example), and the corresponding growth conditions for functions $f$ and $g$ coincide with ours. From [18] it follows that the condition $\lambda_{0}<1+2 / n$ in (6.13) is optimal. The results of [11] and [12] show that $\lambda_{3} \leq 1+1 /(n+1)$ is necessary for the unique solvability if $\Omega=\mathbb{R}^{n}$ and $u^{0} \in L_{1}\left(\mathbb{R}^{n}\right)$. On the other hand, if one deals with particular nonlinearities, then the growth conditions mentioned above need not be optimal: see [9], where the authors consider nonnegative solutions of

$$
\partial_{t} u-\Delta u=-\vec{a} \cdot \nabla\left(u^{q}\right)-u^{p}+M \delta_{0} \otimes \delta_{0} \quad \text { in } \mathbb{R}^{n} \times \mathbb{R}^{+},
$$

where $\vec{a}$ is a constant vector, $p, q>1$, and $M>0$ (cf. also Theorem 7.7).

Having concentrated on the case $r=1$, we now consider the other extreme, namely $r \rightarrow \infty$.

Proposition 6.6. Suppose that the $\lambda_{j}>1$ satisfy

$$
\begin{array}{lll}
\lambda_{0}<1+2 /(n-2), & \lambda_{1}<1+2 /(n-1), & \\
\lambda_{2}<1+1 /(n-2), & \lambda_{3}<1+1 /(n-1), & \lambda_{4}<1+1 /(n-2)
\end{array}
$$

and that (6.14) and (6.15) hold. Then there exist $q \in(1, n /(n-1))$ and $s_{j}$, belonging to $\left[1,2-n / q^{\prime}\right)$ for $0 \leq j \leq 4$, such that (6.16) has for each

$$
\left(u^{0},\left(\mu_{\Omega}, \mu_{1}\right)\right) \in W_{q, \mathcal{B}}^{2} \times L_{\infty, \mathrm{loc}}\left(\mathbb{R}^{+}, \mathcal{M} \times \mathcal{M}\left(\Gamma_{1}\right)\right) .
$$

a unique maximal $C\left(W_{q}^{\vec{s}}\right)$-solution $u$ satisfying $u(0)=u^{0}$. Furthermore, $u$ is nonnegative if $\left(u^{0},\left(\mu_{\Omega}, \mu_{1}\right)\right) \geq 0$. 
Proof. Since $\lambda_{j}^{*}(n, r, \infty)$ tends towards the upper bound for $\lambda_{j}$ in (6.17) as $r \rightarrow \infty$, we can fix $r$ so large that $\lambda_{j}<\lambda_{j}^{*}(n, r, \infty)$ for $0 \leq j \leq 4$. Thus we deduce from Re$\operatorname{mark6.2(a)}$ that we can find $q \in(1, n /(n-1))$ and $s_{j} \in\left[1,2-n / q^{\prime}\right)$ for $0 \leq j \leq 4$ such that (6.7) and (6.8) are satisfied. By increasing $r$ further, if necessary, we can also assume that $r>2 /\left(2-n / q^{\prime}-s_{j}\right)$ for $0 \leq j \leq 4$. Now the assertion follows from Remark 6.2 b) and (the corresponding extension of) Corollary 6.4.

Note that $u$ is, in fact, a $C^{\rho}\left(W_{q}^{1}\right)$-solution of (6.16) for a sufficiently small $\rho>0$.

We recall that, in Propositions 6.5 and 6.6. the assumption that $\left(\mu_{\Omega}, \mu_{1}\right)$ be independent of $u$ has been made for simplicity only.

\section{INTEGRABLE DATA}

Now we consider initial boundary value problems with integrable data. For simplicity, we restrict our considerations to

$$
\begin{aligned}
& \partial_{t} u+\mathcal{A} u=f(x, t, u) \quad \text { in } \quad \Omega \times(0, \infty), \\
& \mathcal{B} u=0 \quad \text { on } \Gamma \times(0, \infty), \\
& u(\cdot, 0)=u^{0} \quad \text { on } \Omega \text {. }
\end{aligned}
$$

The general theorem. We suppose that

- $1<q<\infty, q \vee(n / 2)<\nu \leq \infty, 1 \leq r<\infty, 1<\lambda<\infty$;

- $n / q+2 / r>2$;

- $\kappa: \mathbb{R}^{+} \rightarrow \mathbb{R}^{+}$is increasing, $\beta \in L_{\nu}$;

- $f \in \operatorname{Car}^{1}\left(\left(\Omega \times \mathbb{R}^{+}\right) \times \mathbb{R}, \mathbb{R}\right)$ with $f_{0}:=f(\cdot, \cdot, 0) \in L_{r, \operatorname{loc}}\left(\mathbb{R}^{+}, L_{q}\right)$;

- $\left|\partial_{3} f(x, t, \xi)\right| \leq \kappa(t)\left(1+\beta(x)|\xi|^{\lambda-1}\right)$ for $(x, t, \xi) \in \Omega \times \mathbb{R}^{+} \times \mathbb{R}$.

In this case the following existence and uniqueness theorem is valid, where we put

$$
\lambda^{\bullet}:=\lambda^{\bullet}(q, n, r, \nu):=\frac{n / q+2 / r-n / \nu}{n / q+2 / r-2}=1+\frac{2-n / \nu}{n / q+2 / r-2} .
$$

Theorem 7.1. Suppose that (7.2) is satisfied and

$$
2 / r^{\prime}<s<2 \wedge(n / q), \quad s, 2 / r^{\prime} \notin\{1 / q, 1+1 / q\} .
$$

Also assume that

$$
0 \leq \frac{n}{n / q+2 / r-2}\left[\left(\frac{1}{q}-\frac{1}{\nu}\right)-\lambda\left(\frac{1}{q}-\frac{s}{n}\right)\right]<\lambda^{\bullet}-\lambda .
$$

If $\Omega$ is unbounded, then assume, in addition, that

$$
\frac{\lambda}{q} \geq \frac{s}{n}+\frac{1}{q}-\frac{1}{\nu}
$$

Then (17.1) has for each $u^{0} \in W_{q, \mathcal{B}}^{2 / r^{\prime}}$ a unique maximal $L_{r \lambda}\left(W_{q}^{s}\right)$-solution.

Proof. One verifies that the strict inequality in (7.4) is equivalent to

$$
r \lambda(s-2+2 / r)<2 .
$$

Moreover, (7.3) implies $s-2+2 / r>0$. Hence we can fix $\sigma \in(s, 2)$ with $\sigma-2 / r$ belonging to $I_{q}$ if $r>1$, such that

$$
p:=r \lambda<2 /(s-\sigma+2 / r) \text {. }
$$


Proposition 15.4 (with $\kappa:=q$ ), the left inequality in (7.4), and (7.5) guarantee that

$$
f^{\natural} \in C_{b}^{1-}\left(L_{r \lambda, \text { loc }}\left(\mathbb{R}^{+}, W_{q, \mathcal{B}}^{s}\right), L_{r, \text { loc }}\left(\mathbb{R}^{+}, L_{q}\right)\right) .
$$

Thus, setting $\mu(u):=f^{\natural}(u)+u^{0} \otimes \delta_{0}$, the assertion follows from Theorems 3.2 and 3.3 .

Henceforth, we find it convenient to use the following notation: $b:=a^{+}$(or $b:=a^{-}$), where $a \in \mathbb{R}$, means that $b>a$ (or $b<a$ ) and $|a-b|$ is sufficiently small.

Remarks 7.2. (a) Suppose that $q, r, \lambda<\lambda^{\bullet}$, and $\nu$ satisfy the assumptions of (7.2) and

$$
\frac{1}{q}<\frac{\lambda}{n}\left(\frac{n}{q}+\frac{2}{r}-2\right)+\frac{1}{\nu}
$$

Then there exists $s \in\left(2 / r^{\prime}, 2 \wedge(n / q)\right)$ such that $\lambda(1 / q-s / n)=1 / q-1 / \nu$. Hence condition (7.4) is satisfied for this choice of $s$.

Proof. Observe that

$$
\left\{1 / q-s / n ; 2 / r^{\prime}<s<2 \wedge(n / q)\right\}=\left((1 / q-2 / n)_{+},(1 / q+2 / r n-2 / n)\right) .
$$

Since

$$
(1 / q-1 / \nu) / \lambda>(1 / q-1 / \nu) / \lambda^{\bullet} \geq(1 / q-2 / n)_{+},
$$

the assertion follows.

(b) Clearly, $\lambda^{\bullet}(q, n, r, \nu)$ is an increasing function of $r$ and $\nu$, and

$$
1<\lambda^{\bullet}(q, n, 1, \nu)=1+(2 / n-1 / \nu) q \leq 1+2 q / n=\lambda^{\bullet}(q, n, 1, \infty)
$$

and

$\lambda^{\bullet}(q, n, \infty, \nu)=1+(2-n / \nu) /(n / q-2) \leq 1+2 q /(n-2 q)=\lambda^{\bullet}(q, n, \infty, \infty)$.

(c) Suppose that $\lambda=1^{+}, \nu=\infty$, and

$$
r<\rho<\frac{q r(n+2)}{r(n-2 q)+2 q}
$$

with $\rho \geq q$ if $\Omega$ is unbounded. Then the solution $u$ belongs to $L_{\rho}\left(\Omega \times J_{T}\right)$ for $T \in J(u)$.

Proof. Put $\sigma:=2^{-}$and $s:=2(1+1 / \rho-1 / r)^{-}$and apply Theorem 3.2 with $p:=\rho$ to obtain a unique maximal $L_{\rho}\left(W_{q}^{s}\right)$-solution. Since $W_{q, \mathcal{B}}^{s} \hookrightarrow L_{\rho}$, the assertion follows.

(d) If $r(n-2 q) \geq n q$, then the upper bound for $\rho$ in (7.6) is not greater than $r$ (which is the lower bound for $\rho$ ). In this case

$$
u \in L_{p}\left(J, W_{q, \mathcal{B}}^{2^{-}}\right) \hookrightarrow L_{\widetilde{p}}(\Omega \times J), \quad \widetilde{p}:=\left(\frac{n q}{n-2 q}\right)^{-},
$$

by choosing $p=: r^{+}$.

(e) If $r=1$, then we can add $\mu_{\Omega} \in \mathcal{M}_{\mathrm{loc}}\left(\mathbb{R}^{+}, L_{q}\right)$ to the right-hand side of the first equation in (7.1). Moreover, $\mu_{\Omega}$ may also depend nonlinearly on $u$.

The exponent appearing on the right-hand side of (7.6) is well-known in the linear theory. An analogous regularity result is also known in the quasilinear case (for equations involving the $p$-Laplacian and a linear right-hand side, for example); see [15] Introduction and Theorem 1.3]. 
A comparison principle. Similarly as in the preceding sections, we can prove a comparison principle for (7.1).

Theorem 7.3. Let the hypotheses of Theorem 7.1 be satisfied. Suppose that u,v belong to $L_{r \lambda, \text { loc }}\left(J, W_{q, \mathcal{B}}^{s}\right)$ and satisfy

$$
\begin{aligned}
& \partial_{t}(u-v)+\mathcal{A}(u-v)=F(u)-F(v)+f^{*} \quad \text { in } \quad \Omega \times(0, \infty), \\
& \mathcal{B}(u-v)=0 \quad \text { on } \quad \Gamma \times(0, \infty), \\
& (u-v)(\cdot, 0)=u^{*} \quad \text { on } \Omega \text {, }
\end{aligned}
$$

where

$$
\left(u^{*}, f^{*}\right) \in W_{q, \mathcal{B}}^{2 / r^{\prime}} \times L_{r, \text { loc }}\left(J, L_{q}\right) .
$$

If $\left(u^{*}, f^{*}\right) \geq 0$, then $u \geq v$.

Proof. This follows once more from Theorem 19.4 by the arguments of the proof of Theorem 5.3.

Corollary 7.4. Let the hypotheses of Theorem 7.1 be satisfied. If $\left(u^{0}, f_{0}\right) \geq 0$, then the solution is positive.

For simplicity, we restricted our considerations to the case where $\mathcal{B}(u-v)=0$. Similarly as in Theorem 6.3 this assumption can be replaced by $\mathcal{B}(u-v)=g^{*}$, provided $g^{*}$ satisfies appropriate regularity conditions. Details are left to the reader.

It is also clear that we can formulate and prove an analogue to Theorem [5.5. We leave this to the reader as well.

Model problems. Theorem 7.1 applies, in particular, to the model problem on $\mathbb{R}^{n}$

$$
\begin{aligned}
\partial_{t} u-\Delta u & =V(x)|u|^{\lambda-1} u+f_{0}(x, t) & & \text { in } \quad \mathbb{R}^{n} \times(0, \infty), \\
u(\cdot, 0) & =0 & & \text { on } \quad \mathbb{R}^{n} .
\end{aligned}
$$

Our next result shows that in this case the growth restriction (7.4) for $\lambda$ is optimal, in general (except, possibly, for the equality sign).

In the following theorem and its proof we denote by $\mathbf{1}_{\rho}$ the characteristic function of $\mathbb{B}_{\rho}:=\left\{x \in \mathbb{R}^{n} ;|x|<\rho\right\}$, and $\mathbf{1}:=\mathbf{1}_{1}$.

Theorem 7.5. Set $\Omega:=\mathbb{R}^{n}$ and let $\nu, r \in[1, \infty)$ and $q \in(1, \infty)$ be fixed such that $\nu>q \vee(n / 2)$ and $n / q+2 / r>2$. Put $\ell:=(n / \nu)^{-}, \alpha:=(n / q)^{-}$, and $\beta:=(1 / r)^{-}$, and define $V \in L_{\nu}$ and $f_{0} \in L_{r, \text { loc }}\left(\mathbb{R}^{+}, L_{q}\right)$ by

$$
V(x):=|x|^{-\ell} \mathbf{1}(x), \quad f_{0}(x, t):=|x|^{-\alpha} \mathbf{1}(x) t^{-\beta}, \quad(x, t) \in \mathbb{R}^{n} \times(0, \infty) .
$$

$$
\lambda>\lambda^{\bullet}(q, n, r, \nu),
$$

then, given any $T>0$, problem (7.7) does not possess any nonnegative weak solution $u \in L_{1, \mathrm{loc}}\left(\Omega \times J_{T}\right)$ satisfying $V u^{\lambda} \in L_{1, \mathrm{loc}}\left(\Omega \times J_{T}\right)$ and the comparison principle of Remark 2.4 (e).

Proof. Set $\psi(x):=|x|^{-\alpha} \mathbf{1}(x)$ and $\varphi(t):=t^{-\beta}$. Let $u \in L_{1, \text { loc }}\left(\Omega \times J_{T}\right)$ be a nonnegative weak solution of (7.7) possessing the stated properties for some $T>0$. We can assume that $T<1$. Due to the comparison principle, we have $u \geq w \vee v$, where $w$ is the solution of (7.7) with $V \equiv 0$, and $v:=u-w$ is a solution of

$$
v_{t}-\Delta v=V u^{\lambda} \quad \text { on } \Omega \times J_{T} .
$$


Denote by $e^{t \Delta}$ the heat (Gauß-Weierstraß) semigroup in $\mathbb{R}^{n}$. Then

$$
\left(e^{s \Delta} \psi\right)(x) \geq \int_{\sqrt{s / 2}<|y|<\sqrt{s}}(4 \pi s)^{-n / 2} e^{-|x-y|^{2} / 4 s}|y|^{-\alpha} d y \geq c s^{-\alpha / 2}
$$

for $|x|<\sqrt{s}<1$.

Let $|x|<\sqrt{t / 2}$ and $t<T$. Then, using (7.9), we obtain

$$
\begin{aligned}
u(x, t) & \geq w(x, t)=\int_{0}^{t}\left(e^{(t-s) \Delta} \psi\right)(x) \varphi(s) d s \\
& \geq \int_{0}^{t-|x|^{2}} c(t-s)^{-\alpha / 2} s^{-\beta} d s \\
& =c t^{1-\alpha / 2-\beta} \int_{0}^{1-|x|^{2} / t}(1-\theta)^{-\alpha / 2} \theta^{-\beta} d \theta \\
& \geq c t^{1-\alpha / 2-\beta} ;
\end{aligned}
$$

hence

$$
u(x, t) \geq c t^{-z} \quad \text { if } \quad|x|<\sqrt{t / 2}, \quad t<T,
$$

where $z:=\alpha / 2+\beta-1$ satisfies

$$
z>(1-\ell / 2) /(\lambda-1),
$$

due to (7.8) and the choice of $\ell, \alpha$, and $\beta$.

Now assume that (7.10) is true for some $z$ satisfying (17.11). Then, assuming $|x|<\sqrt{t}$ and $t<T$, we obtain

$$
\begin{aligned}
u(x, t) & \geq v(x, t)=\int_{0}^{t}\left(e^{(t-s) \Delta} V(\cdot) u^{\lambda}(\cdot, s)\right)(x) d s \\
& \geq c \int_{0}^{t}\left(e^{(t-s) \Delta}\left[|\cdot|^{-\ell} s^{-\lambda z} \mathbf{1} \sqrt{s / 2}\right]\right)(x) d s \\
& \geq c \int_{0}^{t} s^{-\lambda z} \int_{\sqrt{s} / 2<|y|<\sqrt{s / 2}} 4 \pi(t-s)^{-n / 2} e^{-|x-y|^{2} / 4(t-s)}|y|^{-\ell} d y d s \\
& \geq c \int_{t / 4}^{t / 2} s^{-\lambda z-\ell / 2} d s \geq c t^{1-\lambda z-\ell / 2} ;
\end{aligned}
$$

hence (7.10) is satisfied with $\widetilde{z}:=\lambda z+\ell / 2-1$. Due to (7.11) we find $\widetilde{z}>z$, and an obvious bootstrapping argument proves (7.10) for any $z>0$. This contradicts $u \in L_{1, \text { loc }}\left(\mathbb{R}^{n} \times J_{T}\right)$ and concludes the proof.

By using similar estimates as in 23, an obvious modification of the above proof gives a similar result if $\Omega$ is bounded.

Remark 7.6. Above we have restricted ourselves to the most interesting case where $n / q+2 / r>2$. If $n / q+2 / r \leq 2$ and $s>2 / r^{\prime}$, then $s>n / q$, so that $W_{q}^{s}$ embeds in $C_{0}(\bar{\Omega})$. Hence in this case problem (7.1) is uniquely solvable for any function $f$ satisfying the hypotheses of (7.2), without any further restriction on $\lambda>1$. Details are left to the reader.

Special cases of the bound $\lambda^{\bullet}(q, n, r, \nu)$ appear in many papers (see, for example, [28, Corollary A] for the case $q=1$ and $r=\infty$, [10] for $q=1$ and $r=\nu=\infty$, and [27], [18], [17] if $r=1$ and $\nu=\infty)$. In many of those papers borderline cases 
are studied as well (also see [16] Section 6] for $r=\infty, \quad q=n / 2, \quad \lambda=2$, and $\left.V(x)=|x|^{-2}\right)$.

Given additional structural conditions, it is sometimes possible to relax the growth restriction on $f$. We illustrate this by a model problem where, for simplicity, we choose an easy setting.

Theorem 7.7. Let $\Omega$ be bounded and suppose that $1<r<\infty$ with $2 / r^{\prime} \neq 1 / q$,

$$
\lambda\left(\frac{n}{q}+\frac{2}{r}-2\right)<n+2, \quad \lambda\left(\frac{1}{q}-\frac{2}{n}\right)<1 .
$$

Then, given $f_{0} \in L_{r, \text { loc }}\left(\mathbb{R}^{+}, L_{q}\right)$ and $u^{0} \in W_{q, \gamma}^{2 / r^{\prime}}$, the problem

$$
\begin{aligned}
& \partial_{t} u-\Delta u=-|u|^{\lambda-1} u+f_{0} \quad \text { in } \quad \Omega \times(0, \infty) \text {, } \\
& u=0 \quad \text { on } \Gamma \times(0, \infty), \\
& u(\cdot, 0)=u^{0} \quad \text { on } \Omega
\end{aligned}
$$

has a unique global weak solution.

Proof. Fix $T>0$ and put $J:=J_{T}$. Let $w$ be the solution of

$$
\begin{aligned}
& \partial_{t} w-\Delta w=\left|f_{0}\right| \quad \text { in } \quad \Omega \times J, \\
& w=0 \quad \text { on } \quad \Gamma \times J, \\
& w(\cdot, 0)=\left|u^{0}\right| \quad \text { on } \quad \Omega .
\end{aligned}
$$

It follows from Remarks $7.2(\mathrm{c})$ and $(\mathrm{d})$ and $(7.12)$ and (7.14) that $w^{\lambda} \in L_{s}(Q)$ for some $s=1^{+}$, where $Q:=\Omega \times J$.

Let $\left(u_{k}^{0}\right)$ be a bounded sequence in $W_{z, \gamma}^{2 / z^{\prime}}$ for some $z=1^{+}$with $z>s$ such that $\left|u_{k}^{0}\right| \leq\left|u^{0}\right|$ and $u_{k}^{0} \rightarrow u^{0}$ in $L_{q}$. Also suppose that $\left(f_{0, k}\right)$ is a sequence in $L_{\infty}(Q)$ satisfying $\left|f_{0, k}\right| \leq\left|f_{0}\right|$ and $f_{0, k} \rightarrow f_{0}$ in $L_{r}\left(J, L_{q}\right)$. Let $u_{k}$ be the solution of (7.13) and $w_{k}$ be the solution of (7.14), respectively, with $\left(u^{0}, f_{0}\right)$ replaced by $\left(u_{k}^{0}, f_{0, k}\right)$. It is well-known that $u_{k}$ is well-defined, and the classical comparison principle implies $\left|u_{k}\right| \leq w_{k}$, whereas $w_{k} \leq w$ follows from Theorem[7.3, for example. Thus the sequence $\left(\left|u_{k}\right|^{\lambda}\right)$ is bounded in $L_{s}(Q)$. Consequently, $\left(u_{k}\right)$ is bounded in $W_{s}^{2,1}(Q)$ by classical regularity theory (e.g., [21, Theorem IV.9.1]). Since $W_{s}^{2,1}(Q)$ is compactly embedded in $L_{1}(Q)$, we can assume, by passing to a subsequence, that $u_{k} \rightarrow u$ a.e. and in $L_{1}(Q)$. Since $\left(\left|u_{k}\right|^{\lambda}\right)$ is bounded in $L_{s}(Q)$ and $\left|u_{k}\right|^{\lambda-1} u_{k} \rightarrow|u|^{\lambda-1} u$ a.e., it follows that $\left|u_{k}\right|^{\lambda-1} u_{k} \rightarrow|u|^{\lambda-1} u$ weakly in $L_{s}(Q)$ (e.g., [20, Theorem 13.44]). Thus, passing to the limit in the weak formulation for the solution $u_{k}$, we obtain a weak solution of (7.13). Uniqueness follows by the arguments of [18, Lemma 3].

Observe that condition (7.12) is weaker than $\lambda<\lambda^{\bullet}(q, n, r, \infty)$.

Let $x_{0} \in \Omega$ and set $f_{0}:=\delta_{x_{0}} \otimes v$ with $v \in L_{r}(J)$. Assuming also that $u^{0}$ belongs to $L_{n r^{\prime} /\left(n r^{\prime}-2\right)}$, problem (7.13) is studied in [19], where the main assumption is equivalent to (7.12) with $q=1$ (see (A2) and the last sentence in Remark 2.2 of [19], where $\gamma, q$, and $N$ play the rôles of $\lambda, r$, and $n$, respectively).

\section{HigheR ORDER PROBLEMS}

The abstract results are also applicable to semilinear parabolic equations of order greater than two. For illustration we consider a simple model problem and leave it to the reader to prove more general results along the lines of the preceding sections. 
We denote by $D^{i} u$ the vector of all spatial derivatives of $u$ of order $i$ and set $D^{0} u:=u$. Then we consider

$$
\begin{aligned}
& \partial_{t} u+\Delta^{2} u=\sum_{i=0}^{3} a_{i}\left|D^{i} u\right|^{\lambda_{i}}+\mu_{\Omega} \quad \text { in } \quad \Omega \times(0, \infty), \\
& u=\partial_{\nu} u=0 \quad \text { on } \Gamma \times(0, \infty),
\end{aligned}
$$

where $\mu_{\Omega} \in \mathcal{M}_{\text {loc }}\left(\mathbb{R}^{+}, \mathcal{M}(\Omega)\right), a_{i} \in L_{\infty}$, and

$$
1<\lambda_{i}<(n+4) /(n+i), \quad 0 \leq i \leq 3 .
$$

We put

$$
W_{q, \mathcal{B}}^{s}:=\left\{\begin{array}{cc}
\left\{u \in W_{q}^{s} ; \gamma u=\partial_{\nu} u=0\right\}, & 1+1 / q<s \leq 4, \\
\left\{u \in W_{q}^{s} ; \gamma u=0\right\}, & 1 / q<s<1+1 / q, \\
W_{q}^{s}, & 0 \leq s<1 / q .
\end{array}\right.
$$

We also fix

$$
q:=1^{+} \quad \text { and } \quad \sigma:=4^{-} \quad \text { with } \quad \sigma<4-n / q^{\prime}
$$

and

$$
s_{i}:=\left(4 / \lambda_{i}\right)^{-} \quad \text { with } \quad \sigma-s_{i}>4 / \lambda_{i}^{\prime}
$$

for $0 \leq i \leq 3$. Then $\vec{\lambda}:=\left(\lambda_{0}, \ldots, \lambda_{3}\right)$ and $\vec{s}:=\left(s_{0}, \ldots, s_{3}\right)$.

Theorem 8.1. Given the above hypotheses, problem (8.1) has a unique maximal $L_{\vec{\lambda}}\left(W_{q}^{\vec{s}}\right)$-solution.

Proof. Set $E_{0}:=L_{q}$ and $E_{1}:=W_{q, \mathcal{B}}^{4}$. Also define a linear operator $A_{0}$ in $E_{0}$ by $A_{0} u:=\Delta^{2} u$ for $u \in W_{q, \mathcal{B}}^{4}$. Then $-A_{0}$ generates a strongly continuous analytic semigroup on $E_{0}$, and $D\left(A_{0}\right) \doteq E_{1}$. Denote by $\left[\left(E_{\xi}, A_{\xi}\right) ;-1 \leq \xi \leq 1\right]$ the interpolation extrapolation scale generated by $\left(E_{0}, A_{0}\right)$ and the interpolation functors

$$
(\cdot, \cdot)_{\theta}:= \begin{cases}{[\cdot, \cdot]_{\theta},} & \theta \in\{1 / 4,1 / 2,3 / 4\}, \\ (\cdot, \cdot)_{\theta, q}, & \text { else. }\end{cases}
$$

Then it follows that $E_{\xi} \doteq W_{q, \mathcal{B}}^{4 \xi}$ for $4 \xi \notin \mathbb{Z}+1 / q$, where $W_{q, \mathcal{B}}^{-s}:=\left(W_{q^{\prime}, \mathcal{B}}^{s}\right)^{\prime}$ by means of the $L_{q}$-duality pairing $\langle\cdot, \cdot\rangle$. From this, (8.3), and Sobolev's embedding theorem we deduce that $\mathcal{M}(\Omega) \hookrightarrow W_{q, \mathcal{B}}^{\sigma-4}$.

Set $X:=L_{\vec{\lambda}}\left(J, W_{q, \mathcal{B}}^{\vec{s}}\right)$ and $Y:=L_{1}\left(J, L_{q}\right)$. Since (8.2)-(8.4) imply the inequality $1 / \lambda_{0} q \geq 1 / q-s_{0} / n$, it follows that $W_{q, \mathcal{B}}^{s_{0}} \hookrightarrow L_{\lambda_{0} q}$. Thus we find that

$$
\left\|a_{0}|u|^{\lambda_{0}-1}|v|\right\|_{Y} \leq c\|u\|_{X}^{\lambda_{0}-1}\|v\|_{X}
$$

Analogous estimates hold for $a_{i}|u|^{\lambda_{i}-1}|v|$ for $1 \leq i \leq 3$, as may be verified by the reader. Using this, we infer, by means of the proof of Proposition 15.4, that $F$, defined by

$$
F(u):=\sum_{i=0}^{3} a_{i}\left|D^{i} u\right|^{\lambda_{j}},
$$

belongs to $C^{1-}\left(L_{\vec{\lambda}, \text { loc }}\left(\mathbb{R}^{+}, W_{q, \mathcal{B}}^{\vec{s}}\right), L_{1, \text { loc }}\left(\mathbb{R}^{+}, L_{q}\right)\right)$. Since $F$ is a local map, it satisfies the Volterra property. Now an obvious modification of the proof of Theorem 3.2 gives the assertion. Details are left to the reader. 


\section{Part 3. Systems}

In this part we discuss extensions of the preceding results to systems. First we present an abstract theorem for semilinear parabolic evolution equations with a diagonal principal part. This theorem is then applied to parabolic initial boundary value problems for which the different components of the solution vector do not possess the same regularity. In the last section we describe the modifications which are needed to handle strongly coupled systems.

\section{Abstract systems}

We suppose that

$m \in \mathbb{N} \backslash\{0\}$, and for each $j \in\{1, \ldots, m\}$

- $E_{j, 0}$ is a reflexive Banach space;

- $A_{j, 0}$ is the negative infinitesimal generator of a strongly continuous analytic semigroup on $E_{j, 0}$;

- $\left[\left(E_{j, \xi}, A_{j, \xi}\right) ;-1 \leq \xi \leq 1\right]$ is the interpolation extrapolation scale generated by $\left(E_{j, 0}, A_{j, 0}\right)$ and a fixed choice of admissible interpolation functors $(\cdot, \cdot)_{j, \theta}, 0<\theta<1$;

- $0 \leq \alpha_{j}<\beta_{j}<1$

- there exists an admissible interpolation functor $\{\cdot, \cdot\}_{j, \beta_{j}-\alpha_{j}}$ of exponent $\beta_{j}-\alpha_{j}$ such that

$$
E_{j, \beta_{j}-1} \doteq\left\{E_{j, \alpha_{j}-1}, E_{j, \alpha_{j}}\right\}_{j, \beta_{j}-\alpha_{j}} \text {. }
$$

Then we put

We also assume that

$$
A_{j}:=A_{j, \alpha_{j}-1}, \quad 1 \leq j \leq m .
$$

- $0 \leq m_{0} \leq m_{1} \leq m$;

- $1<p_{j}<1 /\left(\alpha_{j}-\beta_{j}+1\right)$ for $1 \leq j \leq m_{0}$;

- $1<r_{j}<1 /\left(\beta_{j}-\alpha_{j}\right)$ and $1 \leq p_{j}<1 /\left(\alpha_{j}-\beta_{j}+1 / r_{j}\right)$ for $m_{0}+1 \leq j \leq m_{1}$

- $1 /\left(\beta_{j}-\alpha_{j}\right)<r_{j}<\infty$ and $\rho_{j} \in\left[0, \beta_{j}-\alpha_{j}-1 / r_{j}\right)$ for $m_{1}+1 \leq j \leq m$.

Given a perfect subinterval $J$ of $[0, \mathrm{~T}]$ containing 0 , we put

$$
\boldsymbol{X}_{\mathrm{loc}}(J):=\prod_{j=1}^{m_{1}} L_{p_{j}, \mathrm{loc}}\left(J, E_{j, \alpha_{j}}\right) \times \prod_{j=m_{1}+1}^{m} C^{\rho_{j}}\left(J, E_{j, \alpha_{j}}\right)
$$

and

$$
\boldsymbol{X}(J):=\prod_{j=1}^{m_{1}} L_{p_{j}}\left(J, E_{j, \alpha_{j}}\right) \times \prod_{j=m_{1}+1}^{m} B U C^{\rho_{j}}\left(J, E_{j, \alpha_{j}}\right) .
$$

For $\boldsymbol{u} \in \boldsymbol{X}(J)$ we write

$$
\boldsymbol{u}=(\boldsymbol{v}, \boldsymbol{w}), \quad \boldsymbol{v}:=\left(u_{1}, \ldots, u_{m_{0}}\right), \quad \boldsymbol{w}:=\left(u_{m_{0}+1}, \ldots, u_{m}\right)
$$

and

$$
\boldsymbol{A}:=[\boldsymbol{B}, \boldsymbol{C}], \quad \boldsymbol{B}:=\operatorname{diag}\left[A_{1}, \ldots, A_{m_{0}}\right], \quad \boldsymbol{C}:=\operatorname{diag}\left[A_{m_{0}+1}, \ldots, A_{m}\right] .
$$


Then we assume that

- $\boldsymbol{\mu} \in C_{b}^{1-}\left(\boldsymbol{X}([0, \boldsymbol{T}]), \prod_{j=1}^{m_{0}} \mathcal{M}\left([0, \boldsymbol{T}], E_{j, \beta_{j}-1}\right)\right)$;

- $\boldsymbol{f} \in C_{b}^{1-}\left(\boldsymbol{X}([0, \mathrm{~T}]), \prod_{j=m_{0}+1}^{m} L_{r_{j}}\left((0, \mathrm{~T}), E_{j, \beta_{j}-1}\right)\right)$;

- $\boldsymbol{\mu}$ and $\boldsymbol{f}$ possess the Volterra property;

- $\boldsymbol{w}^{0} \in \prod_{j=m_{0}+1}^{m} E_{j, \beta_{j}-1 / r_{j}}$,

and consider the system

$$
\begin{array}{rlrl}
\dot{\boldsymbol{v}}+\boldsymbol{B} \boldsymbol{v} & =\boldsymbol{\mu}(\boldsymbol{v}, \boldsymbol{w}) \quad \text { in } & {[0, \mathrm{~T}],} \\
\dot{\boldsymbol{w}}+\boldsymbol{C} \boldsymbol{w} & =\boldsymbol{f}(\boldsymbol{v}, \boldsymbol{w}) \quad \text { in } \quad & (0, \mathrm{~T}], \\
\boldsymbol{w}(0) & =\boldsymbol{w}^{0}
\end{array}
$$

Setting $\overline{\boldsymbol{\mu}}(\boldsymbol{u}):=\left(\boldsymbol{\mu}(\boldsymbol{u}), \boldsymbol{f}(\boldsymbol{u})+\boldsymbol{w}^{0} \otimes \delta_{0}\right)$, we can rewrite (9.4) as

$$
\dot{\boldsymbol{u}}+\boldsymbol{A} \boldsymbol{u}=\overline{\boldsymbol{\mu}}(\boldsymbol{u}) \quad \text { on }[0, \boldsymbol{T}] .
$$

We also set

$$
\boldsymbol{E}_{\boldsymbol{\xi}}:=\prod_{j=1}^{m} E_{j, \xi_{j}}, \quad \boldsymbol{E}_{\boldsymbol{\xi}}^{\sharp}:=\prod_{j=1}^{m} E_{j, \xi_{j}}^{\sharp}
$$

for $\boldsymbol{\xi}=\left(\xi_{1}, \ldots, \xi_{m}\right) \in[-1,1]^{m}$, as well as

$$
\langle\boldsymbol{\varphi}, \boldsymbol{u}\rangle_{\boldsymbol{\alpha}}:=\sum_{j=1}^{m}\left\langle\varphi_{j}, u_{j}\right\rangle_{\alpha_{j}}, \quad(\boldsymbol{\varphi}, \boldsymbol{u}) \in \boldsymbol{E}_{-\boldsymbol{\alpha}}^{\sharp} \times \boldsymbol{E}_{\boldsymbol{\alpha}} .
$$

Moreover, $A_{j}^{\top}:=A_{j,-\alpha_{j}}^{\sharp}$ for $1 \leq j \leq m$, and

$$
\boldsymbol{A}^{\top}:=\operatorname{diag}\left[A_{1}^{\top}, \ldots, A_{m}^{\top}\right] \in \mathcal{L}\left(\boldsymbol{E}_{\mathbf{1}-\boldsymbol{\alpha}}^{\sharp}, \boldsymbol{E}_{-\boldsymbol{\alpha}}^{\sharp}\right) .
$$

Then $\boldsymbol{u}$ is said to be an $\boldsymbol{X}$-solution of (9.4) on $J$, provided $\boldsymbol{u} \in \boldsymbol{X}_{\text {loc }}\left(J^{*}\right)$ and

$$
\int_{J}\left\langle-\dot{\varphi}+\boldsymbol{A}^{\top} \boldsymbol{\varphi}, \boldsymbol{u}\right\rangle_{\boldsymbol{\alpha}} d t=\int_{J} \boldsymbol{\varphi} d \overline{\boldsymbol{\mu}}(\boldsymbol{u})
$$

for every $\boldsymbol{\varphi} \in C_{c}\left(J^{*}, \boldsymbol{E}_{1-\boldsymbol{\alpha}}^{\sharp}\right) \cap C^{1}\left(J^{*}, \boldsymbol{E}_{-\boldsymbol{\alpha}}^{\sharp}\right)$.

Now we can formulate the following existence, uniqueness, and continuity theorem for system (9.4).

Theorem 9.1. Let assumptions (9.1)-(9.3) be satisfied. Then problem (9.4) has a unique maximal $\boldsymbol{X}$-solution, $\boldsymbol{u}:=\boldsymbol{u}\left(\boldsymbol{\mu}, \boldsymbol{f}, \boldsymbol{w}^{0}\right)$, satisfying

$$
u_{j} \in L_{r_{j}, \mathrm{loc}}\left(J^{*}, E_{j, \alpha_{j}}\right) \cap W_{r_{j}, \text { loc }}^{1}\left(J^{*}, E_{j, \alpha_{j}-1}\right), \quad m_{0}+1 \leq j \leq m .
$$

If $\boldsymbol{u} \in \boldsymbol{X}(J(\boldsymbol{u}))$, then $\boldsymbol{u}$ is global. The map sending $\left(\boldsymbol{\mu}, \boldsymbol{f}, \boldsymbol{w}^{0}\right)$ into $\boldsymbol{u}\left(\boldsymbol{\mu}, \boldsymbol{f}, \boldsymbol{w}^{0}\right)$ is Lipschitz continuous from the spaces occurring in (9.3) into $\boldsymbol{X}([0, \mathrm{~T}])$.

The proof of this theorem is also postponed to the end of Section 14.

Clearly, the Lipschitz continuity of the solution $\boldsymbol{u}(\cdot, \cdot, \cdot)$ is to be understood in the sense made precise in Theorems 1.1, 2.1] and 2.3, respectively. 


\section{WEAKLY COUPLED PARABOLIC SYSTEMS}

It is the purpose of this section to show how Theorem 9.1 can be applied to concrete situations. For this we restrict ourselves to second order systems, for simplicity.

Henceforth, $(\mathcal{A}, \mathcal{B})$ is said to be a strongly uniformly elliptic boundary value problem whenever $(\mathrm{P} 0)$ is satisfied and $\mathcal{A}$ and $\mathcal{B}$ are given by (P1) and (P2), respectively. Of course, if $\left(\mathcal{A}_{1}, \mathcal{B}_{1}\right)$ and $\left(\mathcal{A}_{2}, \mathcal{B}_{2}\right)$ are two such problems, then each one can have its own boundary decomposition, that is, $\Gamma=\Gamma_{1,0} \cup \Gamma_{1,1}$ and $\Gamma=\Gamma_{2,0} \cup \Gamma_{2,1}$, with $\Gamma_{1,0} \neq \Gamma_{2,0}$, in general.

We suppose that

- $0 \leq m_{0} \leq m_{1} \leq m$;

- $\left(\mathcal{A}_{j}, \mathcal{B}_{j}\right)$ is a strongly uniformly elliptic boundary value problem for $1 \leq j \leq m$;

- $1<q_{j}<\infty, s_{j}, \sigma_{j} \in I_{q_{j}}$ with $0 \leq s_{j}<\sigma_{j}<2$ for $1 \leq j \leq m$;

- $1 \leq p_{j}<2 /\left(s_{j}-\sigma_{j}+2\right)$ for $1 \leq j \leq m_{0}$

- $1<r_{j}<2 /\left(\sigma_{j}-s_{j}\right)$ with $\sigma_{j}-2 / r_{j} \in I_{q_{j}}$ and $1 \leq p_{j}<2 /\left(s_{j}-\sigma_{j}+2 / r_{j}\right)$ for $m_{0}+1 \leq j \leq m_{1}$

- $2 /\left(\sigma_{j}-s_{j}\right)<r_{j}<\infty$ and $0 \leq 2 p_{j}<\sigma_{j}-s_{j}-2 / r_{j}$ for $m_{1}+1 \leq j \leq m$.

Then we put

$$
\boldsymbol{X}_{\boldsymbol{p}, \boldsymbol{q}, \mathrm{loc}}^{\boldsymbol{s}}(J):=\prod_{j=1}^{m_{1}} L_{p_{j}, \mathrm{loc}}\left(J, W_{q_{j}, \mathcal{B}_{j}}^{s_{j}}\right) \times \prod_{j=m_{1}+1}^{m} C^{p_{j}}\left(J, W_{q_{j}, \mathcal{B}_{j}}^{s_{j}}\right)
$$

and assume that

- $\mu_{j} \in C_{b}^{1-}\left(\boldsymbol{X}_{\boldsymbol{p}, \boldsymbol{q}, \mathrm{loc}}^{\boldsymbol{s}}\left(\mathbb{R}^{+}\right), \mathcal{M}_{\mathrm{loc}}\left(\mathbb{R}^{+}, W_{q_{j}, \mathcal{B}_{j}}^{\sigma_{j}-2}\right)\right)$ for $1 \leq j \leq m_{0}$;

- $\left(u_{j}^{0}, f_{j}\right) \in W_{q_{j}, \mathcal{B}_{j}}^{\sigma_{j}-2 / r_{j}} \times C_{b}^{1-}\left(\boldsymbol{X}_{\boldsymbol{p}, \boldsymbol{q}, \mathrm{loc}}^{s}\left(\mathbb{R}^{+}\right), L_{r_{j}, \text { loc }}\left(\mathbb{R}^{+}, W_{q_{j}, \mathcal{B}_{j}}^{\sigma_{j}-2}\right)\right)$ for $m_{0}+1 \leq j \leq m$

- $\mu_{j}$ and $f_{k}$ possess the Volterra property.

We denote by $A_{j}$ the $W_{q_{j}, \mathcal{B}_{j}}^{\sigma_{j}-2}$-realization of $\left(\mathcal{A}_{j}, \mathcal{B}_{j}\right)$, that is, $A_{j}$ is the unique extension in $\mathcal{L}\left(W_{q_{j}, \mathcal{B}_{j}}^{\sigma_{j}}, W_{q_{j}, \mathcal{B}_{j}}^{\sigma_{j}-2}\right)$ of $\mathcal{A}_{j} \mid W_{q_{j}, \mathcal{B}_{j}}^{2}$. Then we consider the problem

$$
\begin{array}{rlrl}
\dot{u}_{j}+A_{j} u_{j}=\mu_{j}\left(u_{1}, \ldots, u_{m}\right) & \text { on } \mathbb{R}^{+}, & 1 \leq j \leq m_{0}, \\
\dot{u}_{j}+A_{j} u_{j}=f_{j}\left(u_{1}, \ldots, u_{m}\right) & \text { on }(0, \infty), \\
u_{j}(0)=u_{j}^{0} & & m_{0}+1 \leq j \leq m .
\end{array}
$$

Put $2 \alpha_{j}:=\sigma_{j}$ and $2 \beta_{j}:=s_{j}$ for $1 \leq j \leq m$, and set

$$
E_{j, \xi}:=W_{q_{j}, \mathcal{B}_{j}}^{2 \xi}, \quad \xi \in I_{q_{j}}, \quad 1 \leq j \leq m .
$$

Then it follows that (9.2) is satisfied with $\rho_{j}=p_{j}$ and $\boldsymbol{X}(J)=\boldsymbol{X}_{\boldsymbol{p}, \boldsymbol{q}}^{\boldsymbol{s}}(J)$, where this space has the obvious meaning. Thus assumption (10.2) implies (9.3), and it follows that (10.3) is a particular instance of (9.4). Hence it is clear what is meant

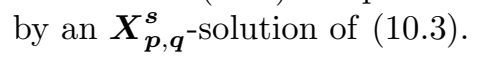


Theorem 10.1. Given assumptions (10.1) and (10.2), problem (10.3) possesses a

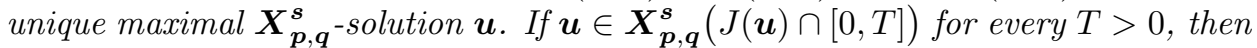
$\boldsymbol{u}$ is global. Moreover, $\boldsymbol{u}$ depends Lipschitz continuously on the data.

Proof. This is a consequence of the preceding observations and Theorem 9.1

It is clear that Remarks 3.5 possess appropriate extensions to system (10.3).

A model problem. As an illustration for Theorem 10.1 we consider the following model problem:

$$
\begin{aligned}
& \left.\begin{array}{l}
\partial_{t} u_{1}+\mathcal{A}_{1} u_{1}=g_{1}\left(x, u_{2}\right) \mu \\
\partial_{t} u_{2}+\mathcal{A}_{2} u_{2}=g_{2}\left(x, u_{1}, u_{2}\right)
\end{array}\right\} \quad \text { in } \Omega \times(0, \infty), \\
& \mathcal{B}_{j} u_{j}=0 \quad \text { on } \Gamma \times(0, \infty), \\
& u_{j}(\cdot, 0)=u_{j}^{0} \quad \text { on } \Omega
\end{aligned}
$$

for $j=1,2$. Here we assume that

- $\Omega$ is bounded and $n \in\{2,3\}$;

- $\left(\mathcal{A}_{j}, \mathcal{B}_{j}\right)$ is a strongly uniformly elliptic boundary value problem for $j=1,2$;

- $n-1<r<\infty$ and $\mu \in L_{r, \text { loc }}\left(\mathbb{R}^{+}, \mathcal{M}\right)$;

- $g_{1} \in C^{0,1}(\bar{\Omega} \times \mathbb{R}, \mathbb{R}), g_{2} \in \operatorname{Car}^{1}\left(\Omega \times \mathbb{R}^{2}, \mathbb{R}\right)$;

- $1<\lambda<2 r /(2+(n-2) r)$;

- $\kappa: \mathbb{R}^{+} \rightarrow \mathbb{R}^{+}$is increasing and $\left|\partial_{2} g_{2}(x, \xi, \eta)\right| \leq c(|\eta|)\left(1+|\xi|^{\lambda-1}\right)$ for $x \in \Omega$ and $\xi, \eta \in \mathbb{R}$.

Note that $r>n-1$ and $n \in\{2,3\}$ guarantee that the upper bound for $\lambda$ is larger than 1.

In general, $u_{1}$ will not be continuous in the variable $x \in \Omega$, due to the singular right-hand side of the first equation of (10.4). On the other hand, in order that $g_{1}\left(x, u_{2}\right) \mu$ be well-defined, $u_{2}$ has to be continuous in $x$. Thus we will look for solutions in

$$
L_{p_{1}}\left(J, W_{q_{1}, \mathcal{B}_{1}}^{s_{1}}\right) \times C\left(J, W_{q_{2}, \mathcal{B}_{2}}^{s_{2}}\right)
$$

for appropriate values of $p_{1}, q_{j}$, and $s_{j}$ such that $s_{2}>n / q_{2}$, which guarantees that $C\left(J, W_{q_{2}, \mathcal{B}_{2}}^{s_{2}}\right) \hookrightarrow C(J, C(\bar{\Omega}))$. For this reason we need a growth restriction for the nonlinearity with respect to the $u_{1}$-variable, whereas no such assumption has to be imposed with respect to the $u_{2}$-variable.

Theorem 10.2. Let (10.5) be satisfied. Suppose that $1<q_{1}<n /(n-2)$, and fix $s_{1}$ such that

$$
n / q_{1}+2 / r-2 / \lambda<s_{1}<2-n+n / q_{1}
$$

and $q_{2} \in(1, \infty)$ with

$$
1 / q_{2} \geq \lambda\left(1 / q_{1}-s_{1} / n\right)
$$

Also fix $s_{2}$ and $\rho$ with

$$
n / q_{2}<s_{2}<2-2 \lambda / r, \quad 0 \leq 2 \rho<2-s_{2}-2 \lambda / r .
$$

Finally, suppose that

$$
2-2 / r \notin \mathbb{N}+1 / q_{1}, \quad 2-2 \lambda / r \notin \mathbb{N}+1 / q_{2} .
$$


Then problem (10.4) has for each

$$
\boldsymbol{u}_{0}:=\left(u_{1}^{0}, u_{2}^{0}\right) \in W_{q_{1}, \mathcal{B}_{1}}^{2-2 / r} \times W_{q_{2}, \mathcal{B}_{2}}^{2-2 \lambda / r}
$$

a unique maximal $L_{r}\left(W_{q_{1}}^{s_{1}}\right) \times C^{\rho}\left(W_{q_{2}}^{s_{2}}\right)$-solution $\boldsymbol{u}\left(\cdot, \boldsymbol{u}^{0}\right)$, and $\boldsymbol{u}\left(\cdot, \boldsymbol{u}^{0}\right)$ is independent of the particular choice of $s_{j}, q_{j}$, and $\rho$. If

$$
\sup _{t \in J(\boldsymbol{u}) \cap[0, T]}\left\|u_{2}\left(t, \boldsymbol{u}^{0}\right)\right\|_{\infty}<\infty \quad \text { for every } T>0,
$$

then $J(\boldsymbol{u})=\mathbb{R}^{+}$.

Proof. First we observe that the upper bound for $\lambda$ in 10.5 implies that we can find $s_{1}$ satisfying (10.6). From this it follows that we can choose $q_{2} \in(1, \infty)$ satisfying (10.7). Thanks to the upper bound for $\lambda$, we also see that condition (10.8) can be satisfied if $1 / q_{2}$ is close to its lower bound in (10.7). Clearly, (10.9) can be satisfied as well.

Put $\boldsymbol{s}:=\left(s_{1}, s_{2}\right), \boldsymbol{q}:=\left(q_{1}, q_{2}\right)$, and $\boldsymbol{p}:=(r, \rho)$ so that

$$
\boldsymbol{X}_{\boldsymbol{p}, \boldsymbol{q}, \text { loc }}^{\boldsymbol{s}}(J)=L_{r, \text { loc }}\left(J, W_{q_{1}, \mathcal{B}_{1}}^{s_{1}}\right) \times C^{\rho}\left(J, W_{q_{2}, \mathcal{B}_{2}}^{s_{2}}\right) .
$$

Given $T>0$, it follows from (10.7), (10.8), and Lemma 4.1 that

$$
\boldsymbol{X}_{\boldsymbol{p}, \boldsymbol{q}}^{\boldsymbol{s}}\left(J_{T}\right) \hookrightarrow L_{r}\left(J_{T}, L_{\lambda q_{2}}\right) \times C\left(J_{T}, C(\bar{\Omega})\right)=: \boldsymbol{Y}_{r, q_{2}}\left(J_{T}\right) .
$$

Set

$$
\boldsymbol{f}(\boldsymbol{u})=\left(f_{1}(\boldsymbol{u}), f_{2}(\boldsymbol{u})\right):=\left(g_{1}^{\natural}\left(u_{2}\right) \mu, g_{2}^{\natural}\left(u_{1}, u_{2}\right)\right) .
$$

It is easily verified that

$$
f_{1} \in C_{b}^{1-}\left(\boldsymbol{Y}_{r, q_{2}}\left(J_{T}\right), L_{r}\left(J_{T}, \mathcal{M}\right)\right), \quad T>0 .
$$

An obvious modification of the proof of Proposition 15.4 shows that

$$
f_{2} \in C_{b}^{1-}\left(\boldsymbol{Y}_{r, q_{2}}\left(J_{T}\right), L_{r / \lambda}\left(J_{T}, L_{q_{2}}\right)\right), \quad T>0 .
$$

Fix $\sigma_{j}$ with $s_{1}<\sigma_{1}<2-n / q_{1}^{\prime}$ and $s_{2}<\sigma_{2}<2-2 \lambda / r$ as well as $\sigma_{1}-2 / r \in I_{q_{1}}$, $\sigma_{2}-2 \lambda / r \in I_{q_{2}}, \quad r<2 /\left(\sigma_{1}-s_{1}\right)$, and $2 \rho<\sigma_{2}-s_{2}-2 \lambda / r$. Then we infer from Lemma 4.1 and (10.12) -10.14 ) that

$$
\boldsymbol{f} \in C_{b}^{1-}\left(\boldsymbol{X}_{\boldsymbol{p}, \boldsymbol{q}}^{\boldsymbol{s}}\left(J_{T}\right), L_{r}\left(J_{T}, W_{q_{1}, \mathcal{B}_{1}}^{\sigma_{1}-2}\right) \times L_{r / \lambda}\left(J_{T}, W_{q_{2}, \mathcal{B}_{2}}^{\sigma_{2}-2}\right)\right), \quad T>0 .
$$

Note that $1<r<2 /\left(s_{1}-\sigma_{1}+2 / r\right)$ and $r / \lambda>2 /\left(\sigma_{2}-s_{2}\right)$. Thus assumptions (10.1) and (10.2) are satisfied with $\left(m_{0}, m_{1}, m\right):=(0,1,2)$ and $\left(r_{1}, r_{2}\right):=(r, r / \lambda)$, and Theorem 10.1 guarantees that problem (10.4) possesses a unique maximal $\boldsymbol{X}_{\boldsymbol{p}, \boldsymbol{q}}^{\boldsymbol{s}}$-solution, $\boldsymbol{u}\left(\cdot, \boldsymbol{u}^{0}\right)$, whenever $\boldsymbol{u}^{0}$ satisfies (10.10). Remarks 3.5 (a) and (b) imply that $\boldsymbol{u}\left(\cdot, \boldsymbol{u}^{0}\right)$ is independent of the particular choice of $\boldsymbol{s}$ and $\boldsymbol{q}$.

Let (10.11) be satisfied. Then

$$
h_{1}:=g_{1}^{\natural}\left(u_{2}\left(\cdot, \boldsymbol{u}^{0}\right)\right) \mu \in L_{r}\left(J(\boldsymbol{u}) \cap J_{T}, \mathcal{M}\right), \quad T>0,
$$

and $u_{1}\left(\cdot, \boldsymbol{u}^{0}\right)$ satisfies the equation

$$
\dot{v}+A_{1} v=h_{1}(t), \quad t \in J(\boldsymbol{u}) \cap J_{T}, \quad v(0)=u_{1}^{0} .
$$

Hence

$$
u_{1}\left(\cdot, \boldsymbol{u}^{0}\right) \in L_{r}\left(J(\boldsymbol{u}) \cap J_{T}, W_{q_{1}, \mathcal{B}_{1}}^{s_{1}}\right), \quad T>0,
$$

by [5, Theorem 4], for example. From this we infer, by the above arguments, that

$$
h_{2}:=g_{1}^{\natural}\left(u_{1}\left(\cdot, \boldsymbol{u}^{0}\right), u_{2}\left(\cdot, \boldsymbol{u}^{0}\right)\right) \in L_{r / \lambda}\left(J(\boldsymbol{u}) \cap J_{T}, L_{q_{2}}\right), \quad T>0 .
$$


Thus, since $u_{2}\left(\cdot, \boldsymbol{u}^{0}\right)$ solves the linear problem

$$
\dot{w}+A_{2} w=h_{2}(t), \quad t \in J(\boldsymbol{u}) \cap J_{T}, \quad w(0)=u_{2}^{0},
$$

we infer from from [5, Proposition 2(iii)] that

$$
u_{2}\left(\cdot, \boldsymbol{u}^{0}\right) \in B U C^{\rho}\left(J(\boldsymbol{u}) \cap J_{T}, W_{q_{2}, \mathcal{B}_{2}}^{s_{2}}\right) .
$$

Hence it follows from Theorem 10.1 that $\boldsymbol{u}\left(\cdot, \boldsymbol{u}^{0}\right)$ is a global solution.

Remarks 10.3. (a) The preceding theorem remains valid if we assume instead of (10.10) that

$$
\boldsymbol{u}^{0} \in W_{q_{1}, \mathcal{B}_{1}}^{\left(s_{1}-2 / r\right)^{+}} \times W_{q_{2}, \mathcal{B}_{2}}^{\left(s_{2}-2 \lambda / r\right)^{+}} .
$$

Proof. This follows from the above proof and from Theorem 10.1 .

(b) It is clear that, by invoking Theorem 9.1, we can also handle systems where the orders of the differential operators differ from component to component of the solution vector.

A system similar to (10.4) has been studied in [22], where it is assumed that $\mu$ is independent of $t$.

\section{Strongly COUpled Systems}

In this section we briefly indicate the modifications for Part2 which are necessary to deal with strongly coupled systems.

We assume that $N$ is a positive integer and interpret all distributions as having values in $\mathbb{R}^{N}$. Thus $W_{q}^{s}:=W_{q}^{s}\left(\Omega, \mathbb{R}^{N}\right)$, etc. Then we suppose that

$$
(\boldsymbol{\alpha}, \mathrm{A}) \in B U C^{\infty}\left(\Omega, \mathbb{R}^{n \times n} \times \mathbb{R}^{N \times N}\right),
$$

and $\boldsymbol{\alpha}=\left[\alpha_{j k}\right]$ is symmetric and uniformly positive definite, and that there exists $\varepsilon>0$ such that

$$
\sigma(\mathrm{A}(x)) \subset\{z \in \mathbb{C} ; \operatorname{Re} z \geq \varepsilon\}, \quad x \in \Omega,
$$

where $\sigma(\cdot)$ denotes the spectrum. Then we set

$$
a_{j k}:=\mathrm{A} \alpha_{j k} \in B U C^{\infty}\left(\Omega, \mathbb{R}^{N \times N}\right), \quad 1 \leq j, k \leq n,
$$

and, using the summation convention with $j$ and $k$ running from 1 to $n$,

$$
\mathcal{A} u:=-\partial_{j}\left(a_{j k} \partial_{k} u\right), \quad u \in \mathcal{D}(\bar{\Omega}) .
$$

We also define the "conormal derivative" by

$$
\partial_{\nu} u:=\nu^{j} \gamma\left(a_{j k} \partial_{k} u\right), \quad u \in \mathcal{D}(\bar{\Omega}),
$$

and set

$$
\mathcal{B} u:=\left\{\begin{aligned}
\gamma u & \text { on } \Gamma_{0}, \\
\partial_{\boldsymbol{\nu}} u & \text { on } \Gamma_{1}
\end{aligned}\right.
$$

Then $(\mathcal{A}, \mathcal{B})$ is a normally elliptic boundary value system (in separated divergence from, using the terminology of [2]).

We write $a^{\top}$ for the transpose of the matrix $a \in \mathbb{R}^{N \times N}$. Then we put

$$
\mathcal{A}^{\sharp} v:=-\partial_{j}\left(a_{j k}^{\top} \partial_{k} v\right), \quad \partial_{\nu}^{\sharp} v:=\nu^{j} \gamma\left(a_{j k}^{\top} \partial_{k} v\right)
$$

for $v \in \mathcal{D}(\bar{\Omega})$ and define $\mathcal{B}^{\sharp}$ by replacing $\partial_{\boldsymbol{\nu}}$ in $(11.2)$ by $\partial_{\boldsymbol{\nu}}^{\sharp}$, so that $\left(\mathcal{A}^{\sharp}, \mathcal{B}^{\sharp}\right)$ is the elliptic boundary value system formally dual to $(\mathcal{A}, \mathcal{B})$. 
The spaces $W_{q, \mathcal{B}}^{s}$ are now defined in analogy to (B.2), except that

$$
W_{q, \mathcal{B}}^{s}:=\left(W_{q^{\prime}, \mathcal{B}^{\sharp}}^{-s}\right)^{\prime}, \quad s \in I_{q} \cap[-2,0) .
$$

We set $E_{0}^{\sharp}:=L_{q^{\prime}}$ and denote by $A_{0}^{\sharp}$ the $L_{q^{\prime}}$-realization of $\left(\mathcal{A}^{\sharp}, \mathcal{B}^{\sharp}\right)$, given by

$$
\operatorname{dom}\left(A_{0}^{\sharp}\right):=W_{q^{\prime}, \mathcal{B}^{\sharp}}^{2}, \quad A_{0}^{\sharp} v:=\mathcal{A}^{\sharp} v .
$$

Then parts (i) and (ii) of Lemma 3.1 remain valid with the modification that $A_{0}^{\prime}=A_{0}^{\sharp}$ (cf. [2]).

Assuming again (3.4) and defining $A$ by (3.5), a (weak) $L_{p}\left(W_{q}^{s}\right)$-solution of (3.6) on $J$ is now an element $u \in L_{p, \text { loc }}\left(J^{*}, W_{q, \mathcal{B}}^{s}\right)$ satisfying

$$
\int_{J}\left\langle-\partial_{t} v+\mathcal{A}^{\sharp} v, u\right\rangle d t=\int_{J} v d \mu(u), \quad v \in \mathcal{D}\left(J^{*}, \mathcal{D}_{\mathcal{B}^{\sharp}}\right),
$$

where $\mathcal{D}_{\mathcal{B}^{\sharp}}:=\left\{v \in \mathcal{D}(\bar{\Omega}) ; \mathcal{B}^{\sharp} v=0\right\}$, of course.

Theorem 11.1. Given the above hypotheses and modifications, all results of Section 3 remain valid if $A$ is induced by a normally elliptic boundary value problem.

Proof. This is obvious from the validity of the analogue to Lemma 3.1

Remarks 11.2. (a) Clearly, the Dirichlet form $\mathfrak{a}$ of $(\mathcal{A}, \mathcal{B})$ is now defined by

$$
\mathfrak{a}(w, z):=\left\langle\partial_{j} w, a_{j k} \partial_{k} z\right\rangle, \quad w, z \in \mathcal{D}(\bar{\Omega}) .
$$

(b) It is also obvious that Theorem 10.1 remains valid if it is supposed that $\left(\mathcal{A}_{j}, \mathcal{B}_{j}\right)$ is a normally elliptic boundary value system for $1 \leq j \leq m$, whose dimension may depend on $j$, of course.

(c) The regularity hypothesis (11.1) has been imposed for convenience only. Everything said above remains true if

$$
(\boldsymbol{\alpha}, \mathrm{A}) \in B U C^{1}\left(\Omega, \mathbb{R}^{n \times n} \times \mathbb{R}^{N \times N}\right)
$$

and $\Gamma$ is a $C^{2}$-submanifold of $\mathbb{R}^{n}$, provided $\mathcal{D}_{\mathcal{B}^{\sharp}}$ is replaced by $W_{q^{\prime}, \mathcal{B}^{\sharp}}^{2-s}$.

(d) Of course, Theorem 10.1 can also be applied if $\left(\mathcal{A}_{j}, \mathcal{B}_{j}\right)$ are suitable strongly coupled elliptic boundary value systems of higher order.

\section{Part 4. Proofs of the Abstract Theorems}

In this part we furnish the proofs for the general theorems presented in Part — and in Section 9

We assume throughout that assumptions (A0) and (A1) are satisfied. For abbreviation, given a perfect subinterval $J$ of $[0, \mathrm{~T}]$ containing 0 , we put

$$
\mathbb{C}_{c}^{1}\left(J^{*},\left(E_{\gamma-1}, E_{\gamma}\right)\right):=C_{c}\left(J^{*}, E_{\gamma}\right) \cap C^{1}\left(J^{*}, E_{\gamma-1}\right) .
$$

We also set

$$
\mathcal{M}_{T}:=\mathcal{M}\left([0, T], E_{\beta-1}\right), \quad 0<T \leq \mathrm{T},
$$

and recall that $J_{T}:=[0, T]$. 


\section{Gluing of solutions}

First we consider the linear case where $\mu$ is independent of $u$, and show that solutions of

$$
\dot{u}+A u=\mu,
$$

defined on adjoining intervals, can be pieced together to yield a solution on the union of these intervals. Since there is no (explicit) initial value in (12.1), this is not immediately clear.

For concise and precise formulations we introduce some further notation. Let $I$ be a nonempty compact interval and $s \in \mathbb{R}$. Denote by $\tau_{s}$ the right translation of functions, that is,

$$
\tau_{s}: E^{I} \rightarrow E^{I+s}, \quad \varphi \mapsto \varphi(\cdot-s)
$$

Then we define the right translation of measures

$$
\tau_{s}: \mathcal{M}\left(I, E_{\beta-1}\right) \rightarrow \mathcal{M}\left(I+s, E_{\beta-1}\right), \quad \mu \mapsto \tau_{s} \mu,
$$

by

$$
\left\langle\tau_{s} \mu, \varphi\right\rangle_{C\left(I+s, E_{1-\beta}^{\sharp}\right)}:=\left\langle\mu, \tau_{-s} \varphi\right\rangle_{C\left(I, E_{1-\beta}^{\sharp}\right)}, \quad \varphi \in C\left(I+s, E_{1-\beta}^{\sharp}\right) .
$$

It is obvious that (12.2) is a linear bijective isometry with $\left(\tau_{s}\right)^{-1}=\tau_{-s}$.

Suppose that $I_{j}:=\left[0, s_{j}\right]$ for $s_{j}>0$ and $j=0,1$, and set $I:=I_{0} \cup\left(s_{0}+I_{1}\right)$. Given $q \in[1, \infty]$ and $v_{j} \in L_{q}\left(I_{j}, E_{\alpha}\right)$ for $j=0,1$, we define $v_{0} \oplus v_{1} \in L_{q}\left(I, E_{\alpha}\right)$ by

$$
v_{0} \oplus v_{1}:=v_{0} \oplus_{s_{0}} v_{1}:=\left\{\begin{array}{cl}
v_{0} & \text { on } I_{0}, \\
\tau_{s_{0}} v_{1} & \text { on } I_{1}+s_{0} .
\end{array}\right.
$$

Below we often simply write $\mu$ for $\mu \mid[0, S]$ if $0<S<T$ and $\mu \in \mathcal{M}_{T}$, if no confusion seems likely.

Assume that $0<T \leq \mathrm{T}$ and $\mu \in \mathcal{M}_{T}$, and let (1.4) be satisfied. Then [5, Theorem 4] guarantees that (12.1) possesses a unique global weak $L_{1}\left(E_{\alpha}\right)$-solution, $u(\mu)$, and

$$
u(\mu) \in \bigcap_{1 \leq p<1 /(\alpha-\beta+1)} L_{p}\left((0, T), E_{\alpha}\right) .
$$

Moreover, from [5. Corollary 2] we know that $u(\mu)$ has a well-defined trace, $u(\mu)(T)$, in $E_{\beta-1}$, and that the map

$$
(\mu \mapsto u(\mu)(T)) \in \mathcal{L}\left(\mathcal{M}_{T}, E_{\beta-1}\right)
$$

is well-defined and uniformly bounded with respect to $T \in(0, \mathrm{~T}]$.

Now we are ready for the proof of the following "gluing lemma".

Lemma 12.1. Let (1.4) be satisfied and suppose that $0<S<T \leq \mathrm{T}$ and $\mu \in \mathcal{M}_{T}$. Denote by $u_{0}$ the weak $L_{1}\left(E_{\alpha}\right)$-solution of (12.1) on $J_{S}$. Set

$$
\mathrm{m}_{T-S}\left(\mu, u_{0}(S)\right):=\tau_{-S}(\mu \mid[S, T])+\left(u_{0}(S)-\mu(\{S\})\right) \otimes \delta_{0} \in \mathcal{M}_{T-S}
$$

and let $u_{1}$ be the weak $L_{1}\left(E_{\alpha}\right)$-solution of

$$
\dot{u}+A u=\mathrm{m}_{T-S}\left(\mu, u_{0}(S)\right) \quad \text { on } J_{T-S} .
$$

Then $u_{0} \oplus u_{1}$ is the weak $L_{1}\left(E_{\alpha}\right)$-solution of (12.1) on $J_{T}$. 
Proof. Suppose that $v \in \mathbb{C}_{c}^{1}\left([0, T),\left(E_{-\alpha}^{\sharp}, E_{1-\alpha}^{\sharp}\right)\right)$. Then

$$
\begin{aligned}
\int_{J_{T}} & \left\langle\left(-\partial+A^{\top}\right) v, u_{0} \oplus u_{1}\right\rangle_{\alpha} d t \\
& =\int_{J_{S}}\left\langle\left(-\partial+A^{\top}\right) v, u_{0}\right\rangle_{\alpha} d t+\int_{J_{T-S}}\left\langle\left(-\partial+A^{\top}\right) \tau_{-S} v, u_{1}\right\rangle_{\alpha} d t .
\end{aligned}
$$

Proposition 1 of [5] and (12.3) imply

$$
\int_{J_{S}}\left\langle\left(-\partial+A^{\top}\right) v, u_{0}\right\rangle_{\alpha} d t=\langle\mu, v\rangle_{C\left(J_{S}, E_{1-\beta}^{\sharp}\right)}-\left\langle v(S), u_{0}(S)\right\rangle_{\beta-1}
$$

and

$$
\begin{gathered}
\int_{J_{T-S}}\left\langle\left(-\partial+A^{\top}\right) \tau_{-S} v, u_{1}\right\rangle_{\alpha} d t=\left\langle\mathrm{m}_{T-S}\left(\mu, u_{0}(S)\right), \tau_{-S} v\right\rangle_{C\left(J_{T-S}, E_{1-\beta}^{\sharp}\right)} \\
=\langle\mu, v\rangle_{C\left([S, T], E_{1-\beta}^{\sharp}\right)}+\left\langle v(S), u_{0}(S)-\mu(\{S\})\right\rangle_{\beta-1} .
\end{gathered}
$$

Note that

$$
\begin{array}{r}
\langle\mu, v\rangle_{C\left(J_{T}, E_{1-\beta}^{\sharp}\right)}=\int_{J_{T}} v d \mu=\int_{J_{S}} v d \mu+\int_{[S, T]} v d \mu-\langle v(S), \mu(\{S\})\rangle_{\beta-1} \\
=\langle\mu, v\rangle_{C\left(J_{S}, E_{1-\beta}^{\sharp}\right)}+\langle\mu, v\rangle_{C\left([S, T], E_{1-\beta}^{\sharp}\right)}-\langle v(S), \mu(\{S\})\rangle_{\beta-1}
\end{array}
$$

for all $v \in \mathbb{C}_{c}^{1}\left([0, T),\left(E_{-\alpha}^{\sharp}, E_{1-\alpha}^{\sharp}\right)\right)$. Consequently, we deduce from (12.4)-12.7) that

$$
\int_{J_{T}}\left\langle\left(-\partial+A^{\top}\right) v, u_{0} \oplus u_{1}\right\rangle_{\alpha} d t=\langle\mu, v\rangle_{C\left(J_{T}, E_{1-\beta}^{\sharp}\right)}, \quad v \in \mathbb{C}_{c}^{1}\left([0, T),\left(E_{-\alpha}^{\sharp}, E_{1-\alpha}^{\sharp}\right)\right) .
$$

Hence $u_{0} \oplus u_{1}$ is the unique weak $L_{1}\left(E_{\alpha}\right)$-solution of (12.1) on $J_{T}$.

\section{Proof of Theorems 1.1 and 1.2}

Assume that $E$ is a Banach space and that $F \hookrightarrow L_{1, \text { loc }}\left(J_{\mathrm{T}}, E\right)$. Then we write $\operatorname{Volt}\left(F, \mathcal{M}_{T}\right)$ for the set of all maps $\mu: F \rightarrow \mathcal{M}_{T}$ possessing the Volterra property. Recall that this means that, given $T \in(0, \mathrm{~T}]$ and $u \in F$,

$$
\mu(u)\left|J_{T}=\mu\left(\left(u \mid J_{T}\right) \oplus 0\right)\right| J_{T} .
$$

We also set

$$
L_{q, T, \gamma}:=L_{q}\left(J_{T}, E_{\gamma}\right), \quad\|\cdot\|_{q, T, \gamma}:=\|\cdot\|_{L_{q}, T, \gamma}
$$

for $0<T \leq T$ and $-1 \leq \gamma \leq 1$, and recall that $\mathbb{B}_{E}$ is the open unit ball in $E$.

We denote by $\mathrm{M}_{T}(\lambda, \omega)$ the set of all $\mu \in \operatorname{Volt}\left(L_{p, T, \alpha}, \mathcal{M}_{T}\right)$ satisfying

$$
\|\mu(0)\|_{\mathcal{M}_{T}} \leq \omega, \quad\|\mu(u)-\mu(v)\|_{\mathcal{M}_{T}} \leq \lambda(R)\|u-v\|_{p, T, \alpha}, \quad u, v \in R \overline{\mathbb{B}}_{p, T, \alpha},
$$

for each $R>0$. Note that

$$
\bigcup_{\lambda, \omega} \mathrm{M}_{T}(\lambda, \omega)=C_{b}^{1-} \cap \operatorname{Volt}\left(L_{p, T, \alpha}, \mathcal{M}_{T}\right) .
$$

Henceforth, $c$ denotes positive constants whose values may differ from occurrence to occurrence, but they are always independent of all free variables of a given situation. 
Proof of Theorem 1.1] Choose $\lambda$ and $\omega$ such that $\mu \in \mathrm{M}_{\mathrm{T}}:=\mathrm{M}_{\mathrm{T}}(\lambda, \omega)$, and fix $R_{0}>0$. Thanks to (13.2), this is possible. Also put $\mathbb{B}_{T}:=\mathbb{B}_{p, T, \alpha},\|\cdot\|_{T}:=\|\cdot\|_{p, T, \alpha}$, and

$$
\mathbf{q}_{T, R}(\nu):=\sup _{v \in R \overline{\mathbb{B}}_{T}}\|\nu(v)\|_{\mathcal{M}_{T}}, \quad \nu \in C_{b}^{1-}\left(L_{p, T, \alpha}, \mathcal{M}_{T}\right) .
$$

(a) Given $v \in R_{0} \overline{\mathbb{B}}_{\mathrm{T}}$, the linear problem $\dot{u}+A u=\mu(v)$ possesses a unique weak $L_{p}\left(E_{\alpha}\right)$-solution $u(\mu(v))$ on $J_{\mathrm{T}}$. From [5. Corollary 3], the Volterra property, and (13.1) we infer that

$$
\|u(\mu(v))\|_{\tau} \leq c \tau^{\varepsilon}, \quad\|u(\mu(v))-u(\mu(w))\|_{\tau} \leq c \tau^{\varepsilon}\|v-w\|_{\tau}
$$

for $v, w \in R_{0} \bar{B}_{\mathrm{T}}, \quad \tau \in \dot{J}_{\mathrm{T}}$, and $\mu \in \mathrm{M}_{\mathrm{T}}$, where $\varepsilon:=\beta-\alpha-1 / p^{\prime}$. Since $\varepsilon>0$, we find $T_{0} \in \dot{J}_{\mathrm{T}}$ such that

$$
\|u(\mu(v))\|_{T_{0}} \leq R_{0}, \quad\|u(\mu(v))-u(\mu(w))\|_{T_{0}} \leq \frac{1}{2}\|v-w\|_{T_{0}}
$$

for $v, w \in R_{0} \overline{\mathbb{B}}_{\mathrm{T}}$ and $\mu \in \mathrm{M}_{\mathrm{T}}$.

Put $\varphi_{\mu}(v):=u(\mu(v \oplus 0))$ for $v \in R_{0} \overline{\mathbb{B}}_{T_{0}}$. Since

$$
L_{p, T_{0}, \alpha} \rightarrow L_{p, \mathrm{~T}, \alpha}, \quad v \mapsto v \oplus 0,
$$

is a linear isometry, it follows from (13.3) that $\varphi_{\mu}$ is for each $\mu \in \mathrm{M}_{\mathrm{T}}$ a contraction mapping $R_{0} \overline{\mathbb{B}}_{T_{0}}$ into itself. Hence Banach's fixed point theorem guarantees the existence of a unique $u_{0}(\mu) \in R_{0} \overline{\mathbb{B}}_{T_{0}}$ satisfying $u_{0}(\mu)=\varphi_{\mu}\left(u_{0}(\mu)\right)$. It is clear that $u_{0}(\mu)$ is a weak $L_{p}\left(E_{\alpha}\right)$-solution of $(\mathrm{EV})$ on $J_{T_{0}}$, and the only one in $R_{0} \overline{\mathbb{B}}_{T_{0}}$.

(b) Suppose that $\nu \in \mathrm{M}_{\mathrm{T}}(\lambda, \omega)$ and $u(\nu)$ is a solution of $\dot{u}+A u=\nu(u)$ on $J_{T}$ for some $T \in(0, \mathrm{~T})$. Fix $R \geq\|u(\nu)\|_{T}$ and set $\mathrm{T}_{1}:=\mathrm{T}-T$. Consider the map

$$
\mathrm{N}_{\mathrm{T}_{1}}(\nu): L_{p, \mathrm{~T}_{1}, \alpha} \rightarrow \mathcal{M}_{\mathrm{T}_{1}}, \quad v \mapsto \mathrm{m}_{\mathrm{T}_{1}}\left(\nu\left(u(\nu) \oplus_{T} v\right), u(\nu)(T)\right),
$$

where $\mathrm{m}_{\mathrm{T}_{1}}$ has been defined in Lemma 12.1 Note that

$$
\left\|\tau_{-T}(\nu(u(\nu) \oplus 0) \mid[T, \mathrm{~T}])\right\|_{\mathcal{M}_{\boldsymbol{T}_{1}}} \leq \omega+R \lambda(R)
$$

and

$$
\left\|\tau_{-T}((\nu(u(\nu) \oplus v)-\nu(u(\nu) \oplus w)) \mid[T, \mathbf{T}])\right\|_{\mathcal{M}_{\mathrm{T}_{1}}} \leq \lambda\left(R+R^{\prime}\right)\|v-w\|_{\mathrm{T}_{1}}
$$

for all $v, w \in R^{\prime} \overline{\mathbb{B}}_{\mathrm{T}_{1}}$. From $u(\nu)=u(\nu(u(\nu)))$ and [5, Corollary 3] we infer that there exists $\kappa \geq 1$ such that

$$
\|u(\nu)(T)\|_{\beta-1} \leq \kappa\|\nu(u(\nu))\|_{\mathcal{M}_{T}} \leq \kappa(\omega+R \lambda(R)) .
$$

Thus, setting

$$
\omega_{1}(R):=3 \kappa(\omega+R \lambda(R)), \quad \lambda_{1}(R):=\lambda(R+\cdot),
$$

it follows that $\omega_{1}(R) \geq \omega$ and $\lambda_{1}(R) \geq \lambda$, as well as

$$
\mathrm{N}_{\mathrm{T}_{1}}(\nu) \in \mathrm{M}_{\mathrm{T}_{1}}\left(\lambda_{1}(R), \omega_{1}(R)\right) .
$$

Note that $\lambda_{1}(R)$ and $\omega_{1}(R)$ are independent of $T \in{\stackrel{\circ}{J_{\top}} \text {. }}$.

(c) Assume $T_{0}<\mathrm{T}$, and put $T:=T_{0}$ and $R:=R_{0}$ in (b). Then it follows from (a) and (b) that there exists $T_{1} \in \dot{J}_{\mathrm{T}-T_{0}}$ such that $\dot{u}+A u=\mathrm{N}_{\mathrm{T}-T_{0}}(\mu)(u)$ has for each $\mu \in \mathrm{M}_{\mathrm{T}}(\lambda, \omega)$ a unique weak $L_{p}\left(E_{\alpha}\right)$-solution $u_{1, \mu}$ on $J_{T_{1}}$ belonging to $R_{0} \overline{\mathbb{B}}_{T_{1}}$.

Set $u_{1}(\mu):=u_{0}(\mu) \oplus u_{1, \mu}$. Then Lemma [2.1 and (12.3) guarantee that $u_{1}(\mu)$ is a weak $L_{p}\left(E_{\alpha}\right)$-solution of $\dot{u}+A u=\mu(u)$ on $J_{T_{0}+T_{1}}$ lying in $2 R_{0} \overline{\mathbb{B}}_{T_{0}+T_{1}}$, and the only one coinciding on $J_{T_{0}}$ with $u_{0}(\mu)$. 
(d) Suppose that $0<T<\mathrm{T}$ and $u \in L_{p, T, \alpha}$ is a weak $L_{p}\left(E_{\alpha}\right)$-solution of (EV) on $J_{T}$. Then we can apply the extension procedure of step (c) to $u$ to obtain a uniquely determined extension to a weak $L_{p}\left(E_{\alpha}\right)$-solution $\bar{u}$ on $J_{S}$ for some $S \in(T, \mathrm{~T}]$, and $\bar{u}$ belongs to $L_{p, S, \alpha}$.

(e) Let $u$ and $v$ be weak $L_{p}\left(E_{\alpha}\right)$-solutions of $(\mathrm{EV})$ on $J_{T}$. Choose $R_{0}>0$ such that $\|u\|_{p, T, \alpha} \vee\|v\|_{p, T, \alpha}<R_{0}$. Then step (a) guarantees that there exists $T_{0} \in \dot{J}_{T}$ such that $u$ and $v$ coincide on $J_{T_{0}}$. Let $T^{*} \in J_{T}$ be the supremum of all $T_{0} \in \dot{J}_{T}$ for which $u=v$ on $J_{T_{0}}$, and suppose that $T^{*}<T$. Since $u \mid J_{T^{*}}$ belongs to $L_{p, T^{*}, \alpha}$, we can apply step (d) to obtain a unique extension of $u \mid J_{T^{*}}$ to a weak $L_{p}\left(E_{\alpha}\right)$-solution on $J_{S}$, where $T^{*}<S \leq T$. Thus $u\left|J_{S}=v\right| J_{S}$, contradicting the maximality of $T^{*}$. Hence $u=v$. This shows that there is at most one maximal solution of (EV).

(f) An obvious argument, based on step (d), now guarantees the existence of a unique maximal solution $u:=u(\mu)$ of $(\mathrm{EV})$. Moreover, $J(u)=J_{\mathrm{T}}$ if $u$ belongs to $L_{p}\left(J(u), E_{\alpha}\right)$, since, otherwise, the extension procedure of step (c) would lead to a contradiction. This proves Theorem 1.1

Proof of Theorem 1.2, For $\varepsilon>0$ we set

$$
\mathrm{U}(\varepsilon):=\left\{\widetilde{\nu} \in \mathrm{M}_{\mathrm{T}}(\lambda, \omega) ; \sup _{\|v\|_{p, \mathrm{~T}, \alpha} \leq R}\|(\widetilde{\nu}-\mu)(v)\|_{\mathcal{M}_{\mathrm{T}}} \leq \varepsilon\right\} .
$$

(a) Fix $T \in J(u(\mu)) \backslash\{0\}$ such that $T<\sup (J(u(\mu)))$ if $u$ is not global. Also fix $R>\|u(\mu)\|_{T}=: R^{*}$. Set $R_{0}:=\left(R-R^{*}\right) / 2$ as well as $\lambda_{1}:=\lambda_{1}(R)$ and $\omega_{1}:=\omega_{1}(R)$. We infer from (a) of the preceding proof that we can find $T_{0}>0$ and $m \in \mathbb{N}$ satisfying $m T_{0}=T$ such that $\dot{u}+A u=\nu(u)$ has for each $\nu \in \mathrm{M}_{T_{0}}\left(\lambda_{1}, \omega_{1}\right)$ a unique weak $L_{p}\left(E_{\alpha}\right)$-solution on $J_{T_{0}}$ belonging to $R_{0} \overline{\mathbb{B}}_{T_{0}}$. We also deduce from (13.3) that

$$
\begin{aligned}
\|u(\nu)-u(\widetilde{\nu})\|_{T_{0}} & \leq\|u(\nu(u(\nu)))-u(\nu(u(\widetilde{\nu})))\|_{T_{0}}+\|u(\nu(u(\widetilde{\nu})))-u(\widetilde{\nu}(u(\widetilde{\nu})))\|_{T_{0}} \\
& \leq \frac{1}{2}\|u(\nu)-u(\widetilde{\nu})\|_{T_{0}}+\|u(\nu(u(\widetilde{\nu})))-u(\widetilde{\nu}(u(\widetilde{\nu})))\|_{T_{0}}
\end{aligned}
$$

for $\nu, \widetilde{\nu} \in \mathrm{M}_{T_{0}}\left(\lambda_{1}, \omega_{1}\right)$. Thus $u(\nu), u(\widetilde{\nu}) \in R_{0} \overline{\mathbb{B}}_{T_{0}}$ and [5, Corollary 3] imply the existence of $c_{1}>0$ such that

$$
\|u(\nu)-u(\widetilde{\nu})\|_{T_{0}} \leq c_{1} \mathrm{q}_{\mathrm{T}, R}(\nu-\widetilde{\nu}), \quad \nu, \widetilde{\nu} \in \mathrm{M}_{\mathrm{T}}\left(\lambda_{1}, \omega_{1}\right) .
$$

Hence there is $\varepsilon_{1}>0$ with

$$
\|u(\nu)\|_{T_{0}} \leq\|u(\mu)\|_{T_{0}}+c_{1} \mathrm{q}_{\mathrm{T}, R}(\nu-\mu) \leq R^{*}+R_{0}=: R_{1}<R
$$

for $\nu \in \mathrm{U}\left(\varepsilon_{1}\right)$, and (13.5)) is true for $\nu, \widetilde{\nu} \in \mathrm{U}\left(\varepsilon_{1}\right)$ as well.

(b) Suppose that $m \geq 2$. It follows from (b) of the preceding proof that

$$
\mathrm{N}_{\mathrm{T}-T_{0}}(\nu) \in \mathrm{M}_{\mathrm{T}-T_{0}}\left(\lambda_{1}, \omega_{1}\right), \quad \nu \in \mathrm{U}\left(\varepsilon_{1}\right) .
$$

Furthermore, (13.5) and (13.6) imply

$$
\begin{aligned}
\| \tau_{-T_{0}}([ & \left.\nu(u(\nu) \oplus v)-\widetilde{\nu}(u(\widetilde{\nu}) \oplus v)] \mid\left[T_{0}, \mathrm{~T}\right]\right) \|_{\mathcal{M}_{\mathrm{T}-T_{0}}} \\
& \leq\|\nu(u(\nu) \oplus v)-\nu(u(\widetilde{\nu}) \oplus v)\|_{\mathcal{M}_{\mathrm{T}}}+\|(\nu-\widetilde{\nu})(u(\widetilde{\nu}) \oplus v)\|_{\mathcal{M}_{\mathrm{T}}} \\
& \leq \lambda(R)\|u(\nu)-u(\widetilde{\nu})\|_{T_{0}}+\mathrm{q}_{\mathrm{T}, R}(\nu-\widetilde{\nu}) \\
& \leq\left(\lambda(R) c_{1}+1\right) \mathbf{q}_{\mathrm{T}, R}(\nu-\widetilde{\nu})
\end{aligned}
$$


for $v \in R_{0} \overline{\mathbb{B}}_{\mathrm{T}-T_{0}}$ and $\nu, \widetilde{\nu} \in \mathrm{U}\left(\varepsilon_{1}\right)$. Similarly,

$$
\begin{aligned}
\left\|u(\nu)\left(T_{0}\right)-u(\widetilde{\nu})\left(T_{0}\right)\right\|_{\beta-1} & \leq \kappa\|\nu(u(\nu))-\widetilde{\nu}(u(\widetilde{\nu}))\|_{\mathcal{M}_{T_{0}}} \\
& \leq \kappa\|\nu(u(\nu))-\nu(u(\widetilde{\nu}))\|_{\mathcal{M}_{T_{0}}}+\kappa \mathrm{q}_{\mathrm{T}, R}(\nu-\widetilde{\nu}) \\
& \leq \kappa \lambda(R)\|u(\nu)-u(\widetilde{\nu})\|_{T_{0}}+\kappa \mathrm{q}_{\mathrm{T}, R}(\nu-\widetilde{\nu}) \\
& \leq \kappa\left(c_{1} \lambda(R)+1\right) \mathrm{q}_{\mathrm{T}, R}(\nu-\widetilde{\nu})
\end{aligned}
$$

for $\nu, \widetilde{\nu} \in \mathrm{U}\left(\varepsilon_{1}\right)$. From this we infer that there exists $\kappa_{1}>0$ such that

$$
\mathrm{q}_{\mathrm{T}-T_{0}, R_{0}}\left(\mathrm{~N}_{\mathrm{T}-T_{0}}(\nu)-\mathrm{N}_{\mathrm{T}-T_{0}}(\widetilde{\nu})\right) \leq \kappa_{1} \mathrm{q}_{\mathrm{T}, R}(\nu-\widetilde{\nu}), \quad \nu, \widetilde{\nu} \in \mathrm{U}\left(\varepsilon_{1}\right) .
$$

Thanks to (13.7) we can apply the arguments of (a) to

$$
\dot{u}+A u=\mathrm{N}_{\mathrm{T}-T_{0}}(\nu)(u)
$$

for $\nu \in \mathrm{U}\left(\varepsilon_{1}\right)$. Thus we find a unique weak $L_{p}\left(E_{\alpha}\right)$-solution $u_{1, \nu}$ of (13.9) belonging to $R_{0} \overline{\mathbb{B}}_{T_{0}}$. From (13.8) and the analogue of (13.5) we deduce that

$$
\left\|u_{1, \nu}-u_{1, \widetilde{\nu}}\right\|_{T_{0}} \leq c_{1} \kappa_{1} \mathbf{q}_{\mathrm{T}, R}(\nu-\widetilde{\nu}), \quad \nu, \widetilde{\nu} \in \mathrm{U}\left(\varepsilon_{1}\right) .
$$

Since

$$
u(\nu)\left|J_{2 T_{0}}=u(\nu)\right| J_{T_{0}} \oplus u_{1, \nu},
$$

we see from (13.5) and (13.10) that, setting $c_{2}:=c_{1}\left(1+\kappa_{1}\right)$,

$$
\|u(\nu)-u(\widetilde{\nu})\|_{2 T_{0}} \leq c_{2} \mathbf{q}_{\mathrm{T}, R}(\nu-\widetilde{\nu}), \quad \nu, \widetilde{\nu} \in \mathrm{U}\left(\varepsilon_{1}\right) .
$$

Hence there exists $\varepsilon_{2} \in\left(0, \varepsilon_{1}\right]$ such that

$$
\|u(\nu)\|_{2 T_{0}} \leq\|u(\mu)\|_{2 T_{0}}+c_{2} \mathrm{q}_{\mathrm{T}, R}(\nu-\mu) \leq R^{*}+R_{0}=R_{1}<R
$$

for $\nu \in \mathrm{U}\left(\varepsilon_{2}\right)$.

(c) Suppose that $2 \leq j \leq m-1$ and put $T_{j}:=j T_{0}$. Also suppose that it has already been shown that there exists $\varepsilon_{j} \in\left(0, \varepsilon_{j-1}\right]$ such that $J(u(\nu)) \supset J_{T_{j}}$ and $u(\nu) \in R_{1} \overline{\mathbb{B}}_{T_{j}}$ for $\nu \in \mathrm{U}\left(\varepsilon_{j}\right)$. Then it follows from (b) of the preceding proof and the arguments used in (b) that

$$
\mathrm{N}_{\mathrm{T}-T_{j}}(\nu) \in \mathrm{M}_{\mathrm{T}-T_{j}}\left(\lambda_{1}, \omega_{1}\right), \quad \nu \in \mathrm{U}\left(\varepsilon_{j}\right),
$$

and that there exists $c_{j}>0$ such that

$$
\mathrm{q}_{\mathrm{T}-T_{j}, R_{0}}\left(\mathrm{~N}_{\mathrm{T}-T_{j}}(\nu)-\mathrm{N}_{\mathrm{T}-T_{j}}(\widetilde{\nu})\right) \leq \kappa_{j} \mathrm{q}_{\mathrm{T}, R}(\nu-\widetilde{\nu}), \quad \nu, \widetilde{\nu} \in \mathrm{U}\left(\varepsilon_{j}\right) .
$$

Thus, similarly as in (b), we find that $J(u(\nu)) \supset J_{T_{j+1}}$ and that there exist constants $c_{j+1}>0$ and $\varepsilon_{j+1} \in\left(0, \varepsilon_{j}\right]$ with

$$
\|u(\nu)-u(\widetilde{\nu})\|_{T_{j+1}} \leq c_{j+1} \mathbf{q}_{\mathrm{T}, R}(\nu-\widetilde{\nu}), \quad \nu, \widetilde{\nu} \in \mathrm{U}\left(\varepsilon_{j}\right),
$$

and

$$
\|u(\nu)\|_{T_{j+1}} \leq\|u(\mu)\|_{T_{j+1}}+c_{j+1} \mathrm{q}_{\mathrm{T}, R}(\nu-\mu) \leq R^{*}+R_{0}=R_{1}<R
$$

for $\nu \in \mathrm{U}\left(\varepsilon_{j+1}\right)$. This implies that we can proceed inductively, so we obtain the assertion for $j=m-1$. 


\section{The CASE of Strong SOlutions}

In this section we prove the remaining abstract theorems of Part 1 . For this we denote by $\Phi_{r, T}(\lambda, \omega)$ the set of all $f \in \operatorname{Volt}\left(L_{p, T, \alpha}, L_{r, T, \beta-1}\right)$ satisfying

$$
\|f(0)\|_{r, T, \beta-1} \leq \omega, \quad\|f(u)-f(v)\|_{r, T, \beta-1} \leq \lambda(R)\|u-v\|_{p, T, \alpha}
$$

for all $u, v \in R \overline{\mathbb{B}}_{p, T, \alpha}$ and $R>0$. Hence

$$
\bigcup_{\lambda, \omega} \Phi_{r, T}(\lambda, \omega)=C_{b}^{1-} \cap \operatorname{Volt}\left(L_{p, T, \alpha}, L_{r, T, \beta-1}\right) .
$$

We also set $\mathbb{B}_{s}:=\mathbb{B}_{E_{s}}$.

Proof of Theorem 2.1. Thanks to (14.2) we can find $\lambda$ and $\omega$ such that $(x, f) \in$ $Y_{\mathrm{T}}:=\omega \overline{\mathbb{B}}_{\beta-1 / r} \times \Phi_{\mathrm{T}}$, where $\Phi_{\mathrm{T}}:=\Phi_{r, \mathrm{~T}}(\lambda, \omega)$. We can also assume that $\gamma$ is close to $\beta$; more precisely, $p<1 /(\alpha-\gamma+1 / r)$.

(a) Fix $R_{0}>0$. Then, given $v \in X_{R_{0}, \mathrm{~T}}:=R_{0} \overline{\mathbb{B}}_{p, \mathrm{~T}, \alpha}$, we denote by $u_{0}(x, f(v))$ the unique strong $L_{r}\left(E_{\gamma}\right)$-solution of the linear Cauchy problem

$$
\dot{u}+A u=f(v) \text { in } \dot{J}_{\mathrm{T}}, \quad u(0)=x,
$$

whose existence is guaranteed by Theorem 5 of $[5]$.

Similarly as in part (a) of the proof of Theorem 1.1, we see, by invoking Proposition 2(ii) of [5], the Volterra property, and (14.1), that there exists $T_{0} \in \dot{J}_{\mathrm{T}}$ such that

$$
\left\|u_{0}(x, f(v))\right\|_{p, T_{0}, \alpha} \leq R_{0}, \quad\left\|u_{0}(x, f(v))-u_{0}(x, f(w))\right\|_{p, T_{0}, \alpha} \leq \frac{1}{2}\|v-w\|_{p, T_{0}, \alpha}
$$

for $v, w \in X_{R_{0}, \mathrm{~T}}$ and $(x, f) \in Y_{\mathrm{T}}$. Thus, setting

$$
\varphi_{(x, f)}(v):=u(x, f(v \oplus 0))=U x+U \star(f(v \oplus 0)), \quad v \in X_{R_{0}, T_{0}},
$$

we infer from (13.4) and the contraction mapping principle that for each $(x, f) \in Y_{\mathrm{T}}$ there exists a unique fixed point $u_{0}(x, f)$ of $\varphi_{(x, f)}$ in $X_{R_{0}, T_{0}}$. Fixing $(x, f)$ and setting $u_{0}:=u_{0}(x, f)$, we see that the Volterra property implies

$$
u_{0}=U x+U \star f\left(u_{0}\right) \quad \text { on } J_{T_{0}} .
$$

Thus, thanks to Theorem 5 of [5], $u_{0}$ is a strong $L_{r}\left(E_{\gamma}\right)$-solution of (2.2), and the only one belonging to $X_{R_{0}, T_{0}}$.

(b) Suppose that $\mathrm{T}_{1}:=\mathrm{T}-T_{0}>0$. From $u_{0}(x, f) \in X_{R_{0}, T_{0}}$ and $f \in \Phi_{\mathrm{T}}$ we infer that

$$
\left\|f\left(u_{0}(x, f)\right)\right\|_{r, T_{0}, \beta-1} \leq c, \quad(x, f) \in Y_{\mathrm{T}} .
$$

Hence [5. Proposition 2(i)] guarantees that

$$
\left\|u_{0}(x, f)\right\|_{\mathbb{W}_{r, T_{0}, \gamma}^{1}} \leq c, \quad(x, f) \in Y_{\mathrm{T}},
$$

where

$$
\mathbb{W}_{r, T, \gamma}^{1}:=L_{r}\left((0, T), E_{\gamma}\right) \cap W_{r}^{1}\left((0, T), E_{\gamma-1}\right) .
$$

Choose $\beta_{1} \neq 1 / r$ and $\gamma_{1}$ satisfying

$$
\gamma>\beta_{1}>\gamma_{1}>\gamma-1 / r^{\prime}, \quad \gamma_{1}>\alpha, \quad p<1 /\left(\alpha-\gamma_{1}+1 / r\right) .
$$

Set $\left(X_{0}, X_{1}\right):=\left(E_{\gamma-1}, E_{\gamma}\right)$ and $\theta:=\beta_{1}-\gamma+1 / r^{\prime}$. Then [4, Lemma 1.1] implies

$$
\left(X_{0}, X_{1}\right)_{\theta, 1} \hookrightarrow E_{\beta_{1}-1 / r} .
$$


Also pick $s$ satisfying $1 / r<s<\gamma-\beta_{1}+1 / r=1-\theta$. Then we deduce from [5] Theorem 3] and (14.5) that

$$
\mathbb{W}_{r, T_{0}, \gamma}^{1} \hookrightarrow C\left(J_{T_{0}}, E_{\beta_{1}-1 / r}\right) .
$$

It follows from (14.4) and (14.6) that there exists $\omega_{1}$ such that

$$
\left\|u_{0}(x, f)\left(T_{0}\right)\right\|_{\beta_{1}-1 / r} \leq \omega_{1}, \quad(x, f) \in Y_{\mathrm{T}} .
$$

Set

$$
\tilde{f}_{x}(v):=\tau_{-T_{0}} f\left(u_{0}(x, f) \oplus v\right), \quad v \in L_{\mathrm{T}_{1}}, \quad(x, f) \in Y_{\mathrm{T}} .
$$

Then $f \in \Phi_{\mathrm{T}}$ and $u_{0}(x, f) \in X_{R_{0}, T_{0}}$ imply that we can choose $\omega_{1}$ such that

$$
\left\|\tilde{f}_{x}(0)\right\|_{r, \mathrm{~T}_{1}, \beta_{1}-1} \leq \omega_{1}, \quad(x, f) \in Y_{\mathrm{T}} .
$$

Similarly, we find that

$$
\left\|\tilde{f}_{x}(v)-\tilde{f}_{x}(w)\right\|_{r, \mathrm{~T}_{1}, \beta_{1}-1} \leq \lambda\left(R_{0}+R\right)\|v-w\|_{p, \mathrm{~T}_{1}, \alpha}, \quad v, w \in X_{R, \mathrm{~T}_{1}},
$$

for $R>0$ and $(x, f) \in Y_{\mathrm{T}}$.

Put $\lambda_{1}:=\lambda\left(R_{0}+\cdot\right)$. Then it follows from (14.7) (14.9) that

$$
\left(u_{0}(x, f)\left(T_{0}\right), \tilde{f}_{x}\right) \in \omega_{1} \overline{\mathbb{B}}_{\beta_{1}-1 / r} \times \Phi_{r, \mathrm{~T}_{1}}\left(\lambda_{1}, \omega_{1}\right), \quad(x, f) \in Y_{\mathrm{T}} .
$$

Since $\alpha<\gamma_{1}<\beta_{1}<\beta$ and $r<1 /(\beta-\alpha)<1 /\left(\beta_{1}-\alpha\right)$, we deduce from (14.10) and step (a) that there exists $T_{1} \in \dot{J}_{\mathrm{T}_{1}}$ such that the Cauchy problem

$$
\dot{u}+A u=\widetilde{f}_{x}(u) \text { in } \dot{J}_{\mathrm{T}_{1}}, \quad u(0)=u_{0}(x, f)\left(T_{0}\right)
$$

has for each $(x, f) \in Y_{\mathrm{T}}$ a unique strong $L_{r}\left(E_{\gamma_{1}}\right)$-solution $v(x, f)$ on $J_{T_{1}}$ belonging to $X_{R_{0}, T_{1}}$. Fixing $(x, f)$ and setting $u_{0}:=u_{0}(x, f)$ and $v:=v(x, f)$, we see that the Volterra property implies

$$
v=U u_{0}\left(T_{0}\right)+U \star \widetilde{f}_{x}(v) \quad \text { on } J_{T_{1}}
$$

(cf. Remark 2.4 f)). Observe that $E_{\gamma-1} \hookrightarrow E_{\gamma_{1}-1}$ and $U \supset U_{\gamma_{1}-1} \supset U_{\gamma-1}$, where $U_{s}$ is the semigroup generated by $-A_{s}$ on $E_{s}$ for $s \in \mathbb{R}$. Hence we can interpret (14.3) as an equation in $E_{\gamma_{1}-1}$. Since (14.11) is also an equation in $E_{\gamma_{1}-1}$, we obtain by inserting $u_{0}\left(T_{0}\right)=U\left(T_{0}\right) x+U \star f\left(u_{0}\right)\left(T_{0}\right)$ in 14.11 that

$$
v(s)=U(s) U\left(T_{0}\right) x+\int_{0}^{T_{0}} U(s) U\left(T_{0}-\tau\right) f\left(u_{0}\right)(\tau) d \tau+\int_{0}^{s} U(s-\tau) \widetilde{f}_{x}(v)(\tau) d \tau
$$

for $s \in J_{T_{1}}$, thanks to the Volterra property of $f$. Thus, by using the Volterra property once more, we find for $T_{0} \leq t \leq T_{0}+T_{1}$ that

$$
\begin{aligned}
v\left(t-T_{0}\right) & =U(t) x+\int_{0}^{T_{0}} U(t-\tau) f\left(u_{0}\right)(\tau) d \tau+\int_{0}^{t-T_{0}} U\left(t-T_{0}-\tau\right) \widetilde{f}_{x}(v)(\tau) d \tau \\
& =U(t) x+\int_{0}^{T_{0}} U(t-\tau) f\left(u_{0} \oplus v\right)(\tau) d \tau+\int_{T_{0}}^{t} U(t-s) f\left(u_{0} \oplus v\right)(s) d s \\
& =U(t) x+U \star f\left(u_{0} \oplus v\right)(t) .
\end{aligned}
$$

Set $u_{1}(x, f):=u_{0}(x, f) \oplus v(x, f)$ for $(x, f) \in Y_{\mathrm{\top}}$. Then $u_{1}(x, f) \in X_{2 R_{0}, T_{0}+T_{1}}$ and the preceding considerations show that

$$
u_{1}(x, f)=U x+U \star f\left(u_{1}(x, f)\right) \quad \text { on } J_{T_{0}+T_{1}},
$$


where this equality holds in $E_{\gamma_{1}-1}$. Since $f \in \Phi_{\mathrm{T}}$ and $u_{1}(x, f) \in X_{2 R_{0}, T_{0}+T_{1}}$, we deduce that

$$
\left\|f\left(u_{1}(x, f)\right)\right\|_{r, T_{0}+T_{1}, \beta-1} \leq c, \quad(x, f) \in Y_{\mathrm{T}} .
$$

Hence we infer from [5, Proposition 2(i)] that

$$
\left\|u_{1}(x, f)\right\|_{\mathbb{W}_{r, T_{0}+T_{1}, \gamma}^{1}} \leq c, \quad(x, f) \in Y_{\mathrm{T}},
$$

and that $u_{1}(x, f)$ is a strong $L_{r}\left(E_{\gamma}\right)$-solution of (2.2) on $J_{T_{0}+T_{1}}$ lying in $X_{2 R_{0}, T_{0}+T_{1}}$, and the only one coinciding on $J_{T_{0}}$ with $u_{0}(x, f)$.

(c) Now the assertions follow by the arguments of steps (d)-(f) of the proof of Theorem 1.1.

Proof of Theorem [2.2, By invoking [5, Proposition 2(ii)] instead of [5, Corollary 3] it is easily verified that the arguments of the proof of Theorem 1.2 yield the assertion in this case also.

Proof of Theorem 2.3. The reader may easily check that the proofs of Theorems 2.1 and 2.2 remain true if the spaces $L_{p}\left(J, E_{\alpha}\right)$ are everywhere replaced by $B U C^{\rho}\left(J, E_{\alpha}\right)$.

For example, denoting by $\|\cdot\|_{\rho, T}$ the norm in $B U C^{\rho}\left(J_{T}, E_{\alpha}\right)$, one obtains the second inequality in (13.3) from the estimate

$$
\begin{aligned}
\|u(x, f(v))-u(x, f(w))\|_{\rho, T} & \leq T^{\rho^{\prime}-\rho}\|u(x, f(v))-u(x, f(w))\|_{\rho^{\prime}, T} \\
& \leq c T^{\rho^{\prime}-\rho}\|f(v)-f(w)\|_{r, T, \beta-1} \\
& \leq c T^{\rho^{\prime}-\rho}\|v-w\|_{\rho, T} \leq \frac{1}{2}\|v-w\|_{\rho, T},
\end{aligned}
$$

where $\rho<\rho^{\prime}<\beta-\alpha-1 / r$ and $T$ is small enough (cf. [5, Proposition 2(iii)]). Notice also that [5. Theorem 3] implies $\mathbb{W}_{r}^{1}\left(J,\left(E_{\gamma-1}, E_{\gamma}\right)\right) \hookrightarrow B U C^{\rho}\left(J, E_{\alpha}\right)$ if $\gamma$ is close to $\beta$.

Proof of Remarks 2.4. (a) The stated conditions are obviously necessary. Since $u=U \star \mu(u)$ (cf. [5]) and $u=U u^{0}+U \star f(u)$, respectively, it follows from Corollary 1 and Proposition 2(i) of [5] that they are sufficient as well.

(b) It is easily verified that the continuation arguments of the proofs of these theorems allow us to construct a solution on $[0, T]$.

(c) Since $u:=u(x, f) \in \mathbb{W}_{r, \text { loc }}^{1}\left(J(u),\left(E_{\gamma-1}, E_{\gamma}\right)\right)$ for $\alpha \leq \gamma<\beta$, it follows from (2.11) that $u \in C\left(J(u),\left(E_{0}, E_{1}\right)_{1 / r^{\prime}, r}\right)$ for $\alpha \leq \gamma<\beta$. Now the assertion is a consequence of the embedding $\left(E_{0}, E_{1}\right)_{\xi, r} \hookrightarrow E_{\eta}$ for $\eta<\xi$.

(d) The assertions concerning Theorems 1.1 and 1.2 follow from the obvious fact that the linear problem $\dot{u}+A u=\mu$ has for each $\mu \in \mathcal{M}_{T}$ a unique solution belonging to $L_{\vec{p}, T, \vec{\alpha}}$, and that [5, Corollary 3] remains valid if $L_{p, T, \alpha}$ is replaced by $L_{\vec{p}, T, \vec{\alpha}}$ and $\varepsilon$ by $\min \left\{1 / p_{j}-1 /\left(\alpha_{j}-\beta+1\right) ; j=0,1, \ldots, m\right\}$. Similar arguments imply the other assertions.

(e) This follows from [5, Proposition 3] and the construction of the solution in the proof of Theorem 1.1 .

Proof of Theorem 9.1. It should be clear by now how obvious adaptions of the preceding proofs yield the asserted results. 


\section{Appendix}

In this appendix we first derive the mapping properties of Nemytskii operators which we used in Sections 5, 8 . Then we present extensions of the results of [5] to the case of nonautonomous principal parts. They are needed for the proof of the positivity theorem which we used in Part 2. In addition, we include some compactness results for solutions of linear evolution equations involving measures and low regularity data. They are required for applying the existence and continuity results of this paper to control problems.

\section{NemYtSkit OPERATORS}

In this section we derive sufficient conditions for the validity of (4.5) in the cases where $F$ and $G$ are Nemytskii operators possessing only a little regularity.

Lemma 15.1. Suppose that

$$
0 \leq t<n / q, \quad 1 \leq r \leq p<\infty, \quad 1 \leq \kappa \leq q .
$$

Put $1 / \pi:=1 / r-1 / p$. Then the following maps are bilinear and continuous, uniformly with respect to $T>0$ :

(i) $L_{\pi}\left(J_{T}, L_{\infty}\right) \times L_{p}\left(J_{T}, W_{q}^{t}\right) \rightarrow L_{r}\left(J_{T}, L_{q}\right), \quad(a, u) \mapsto a u$;

(ii) $L_{\pi}\left(J_{T}, L_{\nu}\right) \times L_{p}\left(J_{T}, W_{q}^{t}\right) \rightarrow L_{r}\left(J_{T}, L_{\kappa}\right), \quad(a, u) \mapsto a u$, if $1 / \kappa-1 / q \leq 1 / \nu \leq 1 / \kappa-1 / q+t / n$, where the lower bound can be replaced by 0 if $\Omega$ is bounded.

(iii) $L_{\pi}\left(J_{T}, L_{\tau}\left(\Gamma_{1}\right)\right) \times L_{p}\left(J_{T}, W_{q}^{t}\right) \rightarrow L_{r}\left(J_{T}, L_{\kappa}\left(\Gamma_{1}\right)\right), \quad(a, u) \mapsto a \gamma_{1} u$, provided $t>1 / q$ and $1 / \tau \leq 1 / \kappa-(n / q-t) /(n-1)$.

Proof. (i) and (ii) follow immediately from Hölder's inequality and Lemma 4.1

(iii) is obtained by similar arguments, using the trace theorem in addition.

We denote by $|\cdot|_{\xi, p}$ and $|\cdot|_{\xi, p, \Gamma}$ the norm in $W_{p}^{\xi}$ and $W_{p}^{\xi}(\Gamma)$, respectively, where $|\cdot|_{p}:=|\cdot|_{0, p}$ and $|\cdot|_{p, \Gamma}:=|\cdot|_{0, p, \Gamma}$

Lemma 15.2. Suppose that $t_{0}, t_{1} \in[0, n / q), 1 \leq r \leq p<\infty, 1 \leq \kappa \leq q$, and $\nu_{0}$, $\nu_{1}, \pi_{0}$, and $\pi_{1}$ belong to $[1, \infty]$. Also suppose that $1<\lambda<\infty$ and

$$
\frac{1}{\pi_{0}}=\frac{1}{r}-\frac{1}{p}, \quad \frac{1}{\pi_{1}}=\frac{1}{r}-\frac{\lambda}{p}, \quad \frac{1}{\kappa}-\frac{1}{q} \leq \frac{1}{\nu_{0}} \leq \frac{1}{\kappa}-\frac{1}{q}+\frac{t_{1}}{n}
$$

as well as

$$
(\lambda-1)\left(\frac{1}{q}-\frac{t_{0}}{n}\right) \leq \frac{1}{\nu_{0}}-\frac{1}{\nu_{1}} \leq \frac{\lambda-1}{q},
$$

where the last upper bound can be omitted if $\Omega$ is bounded.

Finally, assume that

$$
m_{0} \in L_{\pi_{0}}\left(J_{T}, L_{\nu_{0}}+L_{\infty}\right), \quad m_{1} \in L_{\pi_{1}}\left(J_{T}, L_{\nu_{1}}\right),
$$

and $a \in \operatorname{Car}\left(\left(\Omega \times J_{T}\right) \times \mathbb{R}, \mathbb{R}\right)$ satisfies

$$
|a(\cdot, \cdot, \xi)| \leq m_{0}+m_{1}|\xi|^{\lambda-1}, \quad \xi \in \mathbb{R} .
$$

Then $(u, v) \mapsto a^{\natural}(u) v$ maps $L_{p}\left(J_{T}, W_{q}^{t_{0}} \times W_{q}^{t_{1}}\right)$ into $L_{r}\left(J_{T}, L_{\kappa}+L_{q}\right)$, and

$$
\left\|a^{\natural}(u) v\right\|_{L_{r}\left(J_{T}, L_{\kappa}+L_{q}\right)} \leq c\left(1+\|u\|_{L_{p}\left(J_{T}, W_{q}^{t_{0}}\right)}^{\lambda-1}\right)\|v\|_{L_{p}\left(J_{T}, W_{q}^{t_{1}}\right)} .
$$


Proof. Define $a_{1} \in \operatorname{Car}\left(\left(\Omega \times J_{T}\right) \times \mathbb{R}, \mathbb{R}\right)$ by $a_{1}(\cdot, \cdot, \xi):=m_{1}|\xi|^{\lambda-1}$. Then, given $u \in W_{q}^{t_{0}}$, Hölder's inequality implies

$$
\left|a_{1}^{\natural}(u)(t)\right|_{\nu_{0}} \leq\left.\left.\left|m_{1}(\cdot, t)\right|_{\nu_{1}}|| u(t)\right|^{\lambda-1}\right|_{\rho}=\left|m_{0}(\cdot, t)\right|_{\nu_{1}}|u(t)|_{(\lambda-1) \rho}^{\lambda-1},
$$

where $1 / \rho:=1 / \nu_{0}-1 / \nu_{1}$. If $(\lambda-1) \rho<1$, which can occur if $\Omega$ is bounded, then we deduce from Hölder's inequality, applied to $u(t) \cdot 1$, that $|u(t)|_{(\lambda-1) \rho}^{\lambda-1} \leq c|u(t)|_{1}^{\lambda-1}$. Hence it follows from (15.2) and Sobolev's embedding theorem that

$$
\left|a_{1}^{\natural}(u)(t)\right|_{\nu_{0}} \leq c\left|m_{1}(\cdot, t)\right|_{\nu_{1}}|u(t)|_{t_{0}, q}^{\lambda-1}, \quad t \in J_{T} .
$$

Thus, by applying Hölder's inequality once more,

$$
\left\|a_{1}^{\natural}(u)\right\|_{L_{\pi_{0}}\left(J_{T}, L_{\nu_{0}}\right)} \leq c\left\|m_{1}\right\|_{L_{\pi_{1}}\left(J_{T}, L_{\nu_{1}}\right)}\|u\|_{L_{p}\left(J_{T}, W_{q}^{t_{0}}\right)}^{\lambda-1},
$$

because

$$
\frac{1}{\pi_{1}}=\frac{1}{r}-\frac{\lambda}{p}=\frac{1}{\pi_{0}}-\frac{\lambda-1}{p}
$$

Now we infer from Lemma 15.1 and the last condition in (15.1) that

$$
\left\|a_{1}^{\natural}(u) v\right\|_{L_{r}\left(J_{T}, L_{\kappa}\right)} \leq c\|u\|_{L_{p}\left(J_{T}, W_{q}^{t_{0}}\right)}^{\lambda-1}\|v\|_{L_{p}\left(J_{T}, W_{q}^{t_{1}}\right)}
$$

for $(u, v) \in L_{p}\left(J_{T}, W_{q}^{t_{0}} \times W_{q}^{t_{1}}\right)$. Hence the assertion is a consequence of (15.3), (15.4), and Lemma 15.1.

Remark 15.3. If $\pi_{1}=\infty$, then we can assume, by increasing $\lambda$ if necessary, that the first inequality in (15.2) is an equality.

Proof. Suppose that $\lambda<\bar{\lambda}$. Then $\pi_{0}=r \lambda^{\prime}>r \bar{\lambda}^{\prime}=: \bar{\pi}_{0}$. Furthermore, $\nu_{1}>\nu_{0}$ implies $L_{\nu_{1}} \hookrightarrow L_{\nu_{0}}+L_{\infty}$, by Hölder's inequality. Thus, since $|\xi|^{\lambda-1} \leq 1+|\xi|^{\bar{\lambda}-1}$ for $\xi \in \mathbb{R}$, it follows that

$$
m_{0}+m_{1}|\xi|^{\lambda-1} \leq \bar{m}_{0}+m_{1}|\xi|^{\bar{\lambda}-1},
$$

where $\bar{m}_{0}:=m_{0}+m_{1} \in L_{\bar{\pi}_{0}}\left(J_{T}, L_{\nu_{0}}+L_{\infty}\right)$. Now the assertion is obvious.

First we consider Nemytskii operators depending on $u$ only.

Proposition 15.4. Suppose that $0<s<n / q$ and $1 \leq r<\infty$. Also suppose that $\kappa \in[1, q], \quad \nu \in(1, \infty]$, and $\lambda \in(1, \infty)$ with

$$
\lambda\left(\frac{1}{q}-\frac{s}{n}\right) \leq \frac{1}{\kappa}-\frac{1}{\nu}, \quad \frac{\lambda}{q} \geq \frac{s}{n}+\frac{1}{\kappa}-\frac{1}{\nu},
$$

where the lower bound for $\lambda / q$ is to be omitted if $\Omega$ is bounded. Let

$$
f \in \operatorname{Car}^{1}\left(\left(\Omega \times \mathbb{R}^{+}\right) \times \mathbb{R}, \mathbb{R}\right)
$$

satisfy

$$
f(\cdot, \cdot, 0) \in L_{r, \text { loc }}\left(\mathbb{R}^{+}, L_{\kappa}+L_{q}\right) .
$$

Put $1 / \nu_{0}:=1 / \kappa-1 / q+s / n$ and assume that there are

$$
m_{0} \in L_{r \lambda^{\prime}, \text { loc }}\left(\mathbb{R}^{+}, L_{\nu_{0}}+L_{\infty}\right), \quad m_{1} \in L_{\infty, \text { loc }}\left(\mathbb{R}^{+}, L_{\nu}\right)
$$

such that

$$
\left|\partial_{3} f(\cdot, \cdot, \xi)\right| \leq m_{0}+m_{1}|\xi|^{\lambda-1}, \quad \xi \in \mathbb{R} .
$$

Then

$$
f^{\natural} \in C_{b}^{1-}\left(L_{r \lambda, \text { loc }}\left(\mathbb{R}^{+}, W_{q}^{s}\right), L_{r, \text { loc }}\left(\mathbb{R}^{+}, L_{\kappa}+L_{q}\right)\right) .
$$


Proof. By the mean-value theorem,

$$
f(\cdot, \cdot, \xi)-f(\cdot, \cdot, \eta)=\int_{0}^{1} \partial_{3} f(\cdot, \cdot, \tau \xi+(1-\tau) \eta)(\xi-\eta) d \tau
$$

for $\xi, \eta \in \mathbb{R}$. From this we infer that

$$
f^{\natural}(u)-f^{\natural}(v)=\int_{0}^{1}\left(\partial_{3} f\right)^{\natural}(\tau u+(1-\tau) v)(u-v) d \tau .
$$

Hence, setting $a:=\left(\partial_{3} f\right)^{\natural}$, it follows from Lemma 15.2 (with $p:=\lambda r, \pi_{0}:=r \lambda^{\prime}$, $\pi_{1}:=\infty, \nu_{1}:=\nu$, and $\left.t_{0}:=t_{1}:=s\right)$ that, given $T>0$,

$$
\left\|f^{\natural}(u)-f^{\natural}(v)\right\|_{L_{r}\left(J_{T}, L_{\kappa}+L_{q}\right)} \leq c\left(1+\|u\|^{\lambda-1}+\|v\|^{\lambda-1}\right)\|u-v\|
$$

for $u, v \in L_{p}\left(J_{T}, W_{q}^{s}\right)$, where $\|\cdot\|$ denotes the norm in that space. Thus the assertion is implied by (15.5).

Remark 15.5. It should be observed that Proposition 15.4 is true for $1<q<\infty$, that is, without restriction (4.1).

Next consider Nemytskii operators depending on $u$ and $\nabla u$. For $\vec{p}:=\left(p_{0}, \ldots, p_{k}\right)$ and $\vec{s}:=\left(s_{0}, \ldots, s_{k}\right)$ put

$$
L_{\vec{p}, \text { loc }}\left(J, W_{q}^{\vec{s}}\right):=\bigcap_{j=0}^{k} L_{p_{j}}\left(J, W_{q}^{s_{j}}\right) .
$$

Proposition 15.6. Suppose that $s_{j} \in[0, n / q)$ for $j \in\{0, \ldots, 3\}$ with $s_{j} \geq 1$ if $j \geq 1$. Also suppose that $\kappa \in[1, q], \nu_{j} \in(1, \infty]$, and $\lambda_{j} \in(1, \infty)$ satisfy

$$
\begin{aligned}
\lambda_{0}\left(\frac{1}{q}-\frac{s_{0}}{n}\right) & \leq \frac{1}{\kappa}-\frac{1}{\nu_{0}}, & \frac{\lambda_{0}}{q} & \geq \frac{s_{0}}{n}+\frac{1}{\kappa}-\frac{1}{\nu_{0}}, \\
\lambda_{1}\left(\frac{1}{q}-\frac{s_{1}-1}{n}\right) & \leq \frac{1}{\kappa}-\frac{1}{\nu_{1}}+\frac{1}{n}, & \frac{\lambda_{1}}{q} & \geq \frac{s_{1}}{n}+\frac{1}{\kappa}-\frac{1}{\nu_{1}}, \\
\lambda_{2}\left(\frac{1}{q}-\frac{s_{2}}{n}\right) & \leq \frac{1}{\kappa}-\frac{1}{\nu_{2}}-\frac{1}{n}, & \frac{\lambda_{2}}{q} & \geq \frac{s_{2}-1}{n}+\frac{1}{\kappa}-\frac{1}{\nu_{2}}, \\
\lambda_{3}\left(\frac{1}{q}-\frac{s_{3}-1}{n}\right) & \leq \frac{1}{\kappa}-\frac{1}{\nu_{3}}, & \frac{\lambda_{3}}{q} & \geq \frac{s_{3}-1}{n}+\frac{1}{\kappa}-\frac{1}{\nu_{3}},
\end{aligned}
$$

where the second column in (15.6) is to be omitted if $\Omega$ is bounded. Set $t_{1, j}:=s_{j}$ for $j=0,1$, and $t_{1, j}:=s_{j}-1$ for $j=2,3$, and put

$$
1 / \nu_{0, j}:=t_{1, j} / n+1 / \kappa-1 / q, \quad 0 \leq j \leq 3 .
$$

Let $f \in \operatorname{Car}^{1}\left(\left(\Omega \times \mathbb{R}^{+}\right) \times \mathbb{R} \times \mathbb{R}^{n}, \mathbb{R}\right)$ satisfy

$$
f(\cdot, \cdot, 0,0) \in L_{r, \text { loc }}\left(\mathbb{R}^{+}, L_{\kappa}+L_{q}\right)
$$

for some $r \in[1, \infty)$, and assume that there are

$$
\bar{m}_{j} \in L_{\infty, \text { loc }}\left(\mathbb{R}^{+}, L_{\nu_{0}, j}+L_{\infty}\right), \quad m_{j} \in L_{\infty, \text { loc }}\left(\mathbb{R}^{+}, L_{\nu_{j}}\right), \quad 0 \leq j \leq 3,
$$

such that

$$
\begin{aligned}
& \left|\partial_{3} f(\cdot, \cdot, \xi, \eta)\right| \leq \bar{m}_{3}+m_{0}|\xi|^{\lambda_{0}-1}+m_{1}|\eta|^{\lambda_{1}-1}, \\
& \left|\partial_{4} f(\cdot, \cdot, \xi, \eta)\right| \leq \bar{m}_{4}+m_{2}|\xi|^{\lambda_{2}-1}+m_{3}|\eta|^{\lambda_{3}-1}
\end{aligned}
$$

for $(\xi, \eta) \in \mathbb{R} \times \mathbb{R}^{n}$. Then, setting $F(u):=f^{\natural}(u, \nabla u)$,

$$
F \in C_{b}^{1-}\left(L_{r \vec{\lambda}, \mathrm{loc}}\left(\mathbb{R}^{+}, W_{q}^{\vec{s}}\right), L_{r, \mathrm{loc}}\left(\mathbb{R}^{+}, L_{\kappa}+L_{q}\right)\right) .
$$


Proof. Fix $T>0$ and set

$$
a_{j}(\cdot, \cdot, \xi):=\bar{m}_{j}+m_{j}|\xi|^{\lambda_{j}-1}, \quad a_{k}(\cdot, \cdot, \eta):=m_{k}|\eta|^{\lambda_{k}-1}, \quad j=0,2, \quad k=1,3,
$$

for $\xi \in \mathbb{R}$ and $\eta \in \mathbb{R}^{n}$. Also set $t_{0, j}:=s_{j}$ for $j=0,2$, and $t_{0, j}:=s_{j}-1$ for $j=1,3$. Then (15.6) is equivalent to

$$
\left(\lambda_{j}-1\right)\left(\frac{1}{q}-\frac{t_{0, j}}{n}\right) \leq \frac{1}{\nu_{0, j}}-\frac{1}{\nu_{j}} \leq \frac{\lambda_{j}-1}{q}, \quad 0 \leq j \leq 3 .
$$

Also put $\pi_{0, j}:=r \lambda_{j}^{\prime}$ and $\pi_{1, j}:=\infty$ for $0 \leq j \leq 3$. Then

$$
\bar{m}_{j} \in L_{\pi_{0, j}}\left(J_{T}, L_{\nu_{0, j}}+L_{\infty}\right), \quad m_{j} \in L_{\pi_{1, j}}\left(J_{T}, L_{\nu_{j}}\right), \quad 0 \leq j \leq 3 .
$$

Since $\partial_{k} \in \mathcal{L}\left(W_{q}^{s}, W_{q}^{s-1}\right)$ for $s \geq 1$ and $1 \leq k \leq n$, we easily infer from Lemma 15.2 (which is obviously also true if $\xi \in \mathbb{R}$ is replaced by $\eta \in \mathbb{R}^{n}$ ) that $\left(\partial_{3} f\right)^{\natural}(u, \nabla u) v$ and $\left(\partial_{4} f\right)^{\natural}(u, \nabla u) \nabla v$ belong to $L_{r}\left(J_{T}, L_{\kappa}+L_{q}\right)$ and can be estimated by $c(B)\|v\|$ for $u$ in a given bounded subset $B$ of $L_{r \vec{\lambda}}\left(J_{T}, W_{q}^{\vec{s}}\right)$ and $v \in L_{r \vec{\lambda}}\left(J_{T}, W_{q}^{\vec{s}}\right)$, where $\|\cdot\|$ is the norm in that space.

From the mean-value theorem we infer that

$$
F(u)-F(v)=\int_{0}^{1}\left\{\left(\partial_{3} f\right)^{\natural}([u, v](\tau))(u-v)+\left(\partial_{4} f\right)^{\natural}([u, v](\tau))(\nabla u-\nabla v)\right\} d \tau,
$$

where

$$
[u, v](\tau):=\tau(u, \nabla u)+(1-\tau)(v, \nabla v), \quad 0 \leq \tau \leq 1 .
$$

Thus, thanks to (15.7), the assertion follows.

Finally, we investigate Nemytskii operators on $\Gamma_{1}$.

Proposition 15.7. Suppose that $1 / q<s<n / q$ and $1 \leq r<\infty$. Also assume that $\kappa \in[1, q], \quad \nu \in(1, \infty]$, and $\lambda \in(1, \infty)$ satisfy

$$
\lambda\left(\frac{n}{q}-s\right) \leq(n-1)\left(\frac{1}{\kappa}-\frac{1}{\nu}\right)
$$

and that

$$
0 \leq \frac{1}{\rho} \leq \frac{1}{\kappa}-\frac{1}{n-1}\left(\frac{n}{q}-s\right)
$$

Assume that $g \in \operatorname{Car}^{1}\left(\left(\Gamma_{1} \times \mathbb{R}^{+}\right) \times \mathbb{R}, \mathbb{R}\right)$ is such that

$$
g(\cdot, \cdot, 0) \in L_{r, \text { loc }}\left(\mathbb{R}^{+}, L_{\kappa}\left(\Gamma_{1}\right)\right),
$$

and there are

$$
m_{0} \in L_{r \lambda^{\prime}, \mathrm{loc}}\left(\mathbb{R}^{+}, L_{\rho}\left(\Gamma_{1}\right)\right), \quad m_{1} \in L_{\infty, \mathrm{loc}}\left(\mathbb{R}^{+}, L_{\nu}\left(\Gamma_{1}\right)\right)
$$

satisfying

$$
\left|\partial_{3} g(\cdot, \cdot, \xi)\right| \leq m_{0}+m_{1}|\xi|^{\lambda-1}, \quad \xi \in \mathbb{R} .
$$

Then, setting $G:=g^{\natural} \circ \gamma$,

$$
G \in C_{b}^{1-}\left(L_{r \lambda, \text { loc }}\left(\mathbb{R}^{+}, W_{q}^{s}\right), L_{r, \text { loc }}\left(\mathbb{R}^{+}, L_{\kappa}\left(\Gamma_{1}\right)\right)\right) .
$$


Proof. Recall that $\gamma_{1} \in \mathcal{L}\left(W_{q}^{s}, W_{q}^{s-1 / q}\left(\Gamma_{1}\right)\right)$. Hence, given $u \in L_{r \lambda}\left(J_{T}, W_{q, \mathcal{B}}^{s}\right)$ for some $T>0$ and setting $1 / \widetilde{\nu}:=1 / \tau-1 / \nu$ for $1 \leq \tau \leq \nu$, Lemma 4.1(iv) implies

$$
\left.\left.\left|m_{1}(t)\right| \gamma_{1} u(t)\right|^{\lambda-1}\right|_{\tau, \Gamma_{1}} \leq\left|m_{1}(t)\right|_{\nu, \Gamma_{1}}\left|\gamma_{1} u(t)\right|_{(\lambda-1) \widetilde{\nu}, \Gamma_{1}}^{\lambda-1} \leq c\left|m_{1}(t)\right|_{\nu, \Gamma_{1}}|u(t)|_{s, q}^{\lambda-1}
$$

for $t \in J_{T}$, provided

$$
\frac{1}{\tau}-\frac{1}{\nu}=\frac{1}{\widetilde{\nu}} \geq \frac{\lambda-1}{n-1}\left(\frac{n}{q}-s\right) .
$$

Hence, by Hölder's inequality,

$$
\left\|m_{1}\left|\gamma_{1} u\right|^{\lambda-1}\right\|_{L_{r \lambda^{\prime}}\left(J_{T}, L_{\tau}\left(\Gamma_{1}\right)\right)} \leq c\left\|m_{1}\right\|_{L_{\infty}\left(J_{T}, L_{\nu}\left(\Gamma_{1}\right)\right)}\|u\|_{L_{r \lambda}\left(J_{T}, W_{q}^{s}\right)}^{\lambda-1} .
$$

From (15.8) we infer that we can find $\tau$ satisfying (15.13) and

$$
\frac{1}{\tau} \leq \frac{1}{\kappa}-\frac{1}{n-1}\left(\frac{n}{q}-s\right) .
$$

Thus Lemma15.1(iii), with $t:=s$ and $p:=r \lambda$, guarantees that

$$
\left\|m_{1}\left|\gamma_{1} u\right|^{\lambda-1} \gamma_{1} v\right\|_{L_{r}\left(J_{T}, L_{\kappa}\left(\Gamma_{1}\right)\right)} \leq c\|u\|^{\lambda-1}\|v\|
$$

for $u, v \in L_{r \lambda}\left(J_{T}, W_{q, \mathcal{B}}^{s}\right)$, where $\|\cdot\|$ denotes the norm of that space.

Similarly, (15.9), Lemma 4.1(iv), and Hölder's inequality imply

$$
\left\|m_{0} \gamma_{1} v\right\|_{L_{r}\left(J_{T}, L_{\kappa}\left(\Gamma_{1}\right)\right)} \leq\left\|m_{0}\right\|_{L_{r \lambda^{\prime}}\left(J_{T}, L_{\rho}\left(\Gamma_{1}\right)\right)}\|v\|
$$

for $v \in L_{r \lambda}\left(J_{T}, W_{q, \mathcal{B}}^{s}\right)$.

By the mean-value theorem,

$$
G(u)-G(v)=\int_{0}^{1} \partial_{3} g^{\natural}\left(\tau \gamma_{1} u+(1-\tau) \gamma_{1} v\right) d \tau\left(\gamma_{1} u-\gamma_{1} v\right) .
$$

Thus we deduce from (15.11), (15.12), (15.14), and (15.15) that, given a bounded subset $B$ of $L_{r \lambda}\left(J_{T}, W_{q, \mathcal{B}}^{s}\right)$,

$$
\|G(u)-G(v)\|_{L_{r}\left(J_{T}, L_{\kappa}\left(\Gamma_{1}\right)\right)} \leq c(B)\|u-v\|
$$

for $u, v \in B$. Now the assertion follows from (15.10).

Observe that, being local operators, the nonlinear maps of Propositions 15.415 .7 trivially possess the Volterra property.

\section{NonAutonomous EQUATIONS}

Throughout this section we suppose that hypotheses (A0) are satisfied. Moreover, given Banach spaces $X_{0}$ and $X_{1}$ satisfying $X_{1} \stackrel{d}{\hookrightarrow} X_{0}$, we denote by $\mathcal{H}\left(X_{1}, X_{0}\right)$ the set of all $B \in \mathcal{L}\left(X_{1}, X_{0}\right)$ such that $-B$, considered as a linear operator in $X_{0}$ with domain $X_{1}$, generates a strongly continuous analytic semigroup, usually denoted by $\left\{e^{-t B} ; t \geq 0\right\}$, on $X_{0}$.

We denote by $\left[\left(E_{\xi}, A_{\xi}\right) ;-1 \leq \xi \leq 1\right]$ the interpolation extrapolation scale generated by $\left(E_{0}, A_{0}\right)$ and a fixed choice of admissible interpolation functors $(\cdot, \cdot)_{\theta}$, $0<\theta<1$. Then we suppose that

$$
A \in C^{\rho}\left([0, \mathrm{~T}], \mathcal{H}\left(E_{\alpha}, E_{\alpha-1}\right)\right)
$$

for some $\rho \in(0,1)$.

It should be observed that now the map $A$ is independent of the operator $A_{0}$. The latter is employed for constructing the interpolation extrapolation scale of which only some of the spaces $E_{\xi}$ are used. 
Setting $\triangle_{\mathrm{T}}^{*}:=\{(t, \tau) ; 0 \leq \tau<t \leq \mathrm{T}\}$, it is known [3, Corollary II.4.4.2] that there exists a unique parabolic evolution operator

$$
U \in C\left(\triangle_{\mathrm{T}}^{*}, \mathcal{L}\left(E_{\alpha-1}, E_{\alpha}\right)\right) \cap C\left(\triangle_{\mathrm{T}}, \mathcal{L}_{s}\left(E_{\alpha-1}\right)\right),
$$

where $\triangle_{\mathrm{T}}$ is the closure of $\triangle_{\mathrm{T}}^{*}$ in $\mathbb{R}^{2}$, and $\mathcal{L}_{s}\left(E_{\alpha-1}\right)$ is the space $\mathcal{L}\left(E_{\alpha-1}\right)$ endowed with the strong operator topology.

It is not difficult to see that

$$
A^{\top}:=A_{-\alpha}^{\sharp} \in C^{\rho}\left([0, \top], \mathcal{H}\left(E_{1-\alpha}^{\sharp}, E_{-\alpha}^{\sharp}\right)\right) .
$$

Thus, given $T \in(0, T]$, there exists a unique parabolic evolution operator $U_{T}^{\sharp}$ for

$$
\left(t \mapsto A^{\top}(T-t)\right) \in C^{\rho}\left(J_{T}, \mathcal{H}\left(E_{1-\alpha}^{\sharp}, E_{-\alpha}^{\sharp}\right)\right) .
$$

Put

$$
V_{T}(t, \tau):=U_{T}^{\sharp}(T-\tau, T-t), \quad(t, \tau) \in \triangle_{T},
$$

and

$$
U \star f(t):=\int_{0}^{t} U(t, \tau) f(\tau) d \tau, \quad 0 \leq t \leq \mathrm{T}, \quad f \in L_{1}\left((0, \mathrm{~T}), E_{\alpha-1}\right),
$$

as well as

$$
V_{T} \circledast g(t):=\int_{t}^{T} V(\tau, t) g(\tau) d \tau, \quad 0 \leq t \leq T, \quad g \in L_{1}\left((0, T), E_{-\alpha}^{\sharp}\right) .
$$

Lemma 16.1. Suppose that

$$
1<p<1 /(\alpha-\beta+1) .
$$

Then, setting $\varepsilon:=\beta-\alpha-1 / p^{\prime}$, we have

$$
T^{-\varepsilon} V_{T} \circledast \in \mathcal{L}\left(L_{p^{\prime}}\left(J_{T}, E_{-\alpha}^{\sharp}\right), C\left(J_{T}, E_{1-\beta}^{\sharp}\right)\right)
$$

$\mathrm{T}$-uniformly; that is, these linear operators are bounded independently of $T \in(0, T]$.

Proof. Let $X_{0}$ and $X_{1}$ be Banach spaces such that $X_{1} \hookrightarrow X_{0}$, and suppose that $B \in C^{\rho}\left(J_{T}, \mathcal{H}\left(X_{1}, X_{0}\right)\right)$ for some $T \in \dot{J}_{\mathrm{T}}$ and some $\rho \in(0,1)$. Let $U_{B}$ be the parabolic evolution operator for $B$. Then (cf. [3, Theorem II.5.1.1])

$$
\left\|U_{B}(t, \tau)\right\|_{\mathcal{L}\left(X_{j}\right)}+(t-\tau)\left\|U_{B}(t, \tau)\right\|_{\mathcal{L}\left(X_{0}, X_{1}\right)} \leq c, \quad 0 \leq \tau<t \leq T, \quad j=0,1 .
$$

This is the analogue for the nonautonomous case to formula (23) in [5]. Thus, by arguing as in [5], we see that [5. Lemma 1(i)] holds for $U_{B}$. Now we apply this to $\left(X_{0}, X_{1}\right):=\left(E_{-\alpha}^{\sharp}, E_{1-\alpha}^{\sharp}\right)$ and $B:=\left(t \mapsto A^{\top}(T-t)\right)$ to obtain the assertion.

From (16.1) we infer that

$$
\left[V_{T} \circledast\right]^{\prime} \in \mathcal{L}\left(\mathcal{M}\left(J_{T}, E_{\beta-1}\right), L_{p}\left(J_{T}, E_{\alpha}\right)\right) .
$$

The proof of [5, Lemma 6] remains valid in this case also to show that

$$
\left[V_{T} \circledast\right]^{\prime} f=U \star f, \quad f \in L_{1}\left(J_{T}, E_{\beta-1}\right) .
$$

Moreover, (16.2) is independent of $T$ in the sense that

$$
\left[V_{S} \circledast\right]^{\prime} \mid \mathcal{M}\left([0, T], E_{\beta-1}\right)=\left[V_{T} \circledast\right]^{\prime}, \quad 0<T<S \leq \mathrm{T} .
$$

It is also independent of $p \in(1,1 /(\alpha-\beta+1))$ in the obvious sense, because $L_{p}\left(J_{T}, E_{\beta-1}\right) \hookrightarrow L_{q}\left(J_{T}, E_{\beta-1}\right)$ for $1 \leq q<p$. For this reason we put

$$
U \star:=\left[V_{\mathrm{T}} \circledast\right]^{\prime} .
$$


Then Lemma 16.1 implies

$$
T^{-\varepsilon} U \star \in \mathcal{L}\left(\mathcal{M}\left([0, T], E_{\beta-1}\right), L_{p}\left((0, T), E_{\alpha}\right)\right) \quad \text { T-uniformly. }
$$

Lemma 16.2. Corollary 2 and Proposition 1 of [5] remain valid in the nonautonomous case.

Proof. It is not difficult to verify that the trace theorem, that is, Corollary 2 of [5], remains valid.

To show that also Proposition 1 continues to hold, we have to modify its proof (cf. [5, Proposition 1]) by observing that now $w:=\left(-\partial+A^{\top}\right) v$ is no longer infinitely smooth but belongs to $C^{\rho}\left(J_{T}, E_{-\alpha}^{\sharp}\right)$. By invoking [3, Theorem II.1.2.1 and Proposition V.2..5.2] we find again that

$$
v=V(T, \cdot) v(T)+V_{T} \circledast w .
$$

Now the remainder of the proof of [5, Proposition 1] gives the assertion.

Observe that now (12.1) is nonautonomous, usually written as

$$
\dot{u}+A(t) u=\mu \quad \text { on }[0, \mathrm{~T}] .
$$

It is now clear that the proof of [ 5 , Theorem 4] remains valid to show that, given $T \in(0, \mathrm{~T}]$ and $\mu \in \mathcal{M}\left([0, T], E_{\beta-1}\right)$, problem (16.3) has a unique weak $L_{1}\left(E_{\alpha}\right)$ solution $u(\mu)$, namely $u(\mu)=U \star \mu$, and

$$
u(\mu) \in \bigcap_{1 \leq p<1 /(\alpha-\beta+1)} L_{p}\left((0, T), E_{\alpha}\right) .
$$

Suppose that $E_{0}$ is an ordered Banach space. Then $A$ is said to be resolvent positive if $A(t)$ has this property for each $t \in[0, \mathrm{~T}]$.

Proposition 16.3. Let $E_{0}$ be an ordered Banach space and suppose that $A$ is resolvent positive. If $\mu$ is positive, then $u(\mu)$ is positive as well.

Proof. Theorem V.2.7.2 in [3] guarantees that $A^{\top}$ is also resolvent positive. Hence we infer from [3] Theorems II.6.4.1 and II.6.4.2] that $V_{T}$ is positive for $0<T \leq \mathrm{T}$. Now the proof of [5. Proposition 3] applies to show that $\mu \geq 0$ implies $U \star \mu \geq 0$.

\section{Compactness}

We continue to presuppose the hypotheses of the preceding section. Furthermore, given Banach spaces $X$ and $Y$, we denote by $\mathcal{K}(X, Y)$ the space of all compact linear maps from $X$ into $Y$.

Proposition 17.1. Suppose that $A_{0}$ has a compact resolvent and assumption (1.4) is satisfied. Then

$$
(\partial+A)^{-1} \in \mathcal{K}\left(\mathcal{M}\left([0, \mathrm{~T}], E_{\beta-1}\right), L_{p}\left((0, \mathrm{~T}), E_{\alpha}\right)\right) .
$$

Proof. Since $(\partial+A)^{-1}=U \star=\left[V_{\mathrm{T}} \circledast\right]^{\prime}$, it suffices to prove that

$$
V_{\mathrm{T}} \circledast \in \mathcal{K}\left(L_{p^{\prime}}\left((0, \mathrm{~T}), E_{-\alpha}^{\sharp}\right), C\left([0, \mathrm{~T}], E_{1-\beta}^{\sharp}\right)\right) .
$$

Fix $p$ in $(1,1 /(\alpha-\beta+1))$ and $\beta_{1}$ in $(\alpha, \beta)$ satisfying $p<1 /\left(\alpha-\beta_{1}+1\right)$, and set $\varepsilon_{1}:=\beta_{1}-\alpha-1 / p^{\prime}$. Then (16.1) holds with $\beta$ and $\varepsilon$ replaced by $\beta_{1}$ and $\varepsilon_{1}$, respectively. From this and an obvious translation of the independent variable we deduce 
that, setting $V:=V_{\mathrm{T}}$,

$$
\left\|\int_{s}^{t} V(\tau, s) g(\tau) d \tau\right\|_{E_{1-\beta}^{\sharp}} \leq c(t-s)^{\varepsilon_{1}}\|g\|_{L_{p^{\prime}}\left(J_{\top}, E_{-\alpha}^{\sharp}\right)}
$$

for $0 \leq s<t \leq \mathrm{T}$. We also observe that

$$
V(\tau, s)=V(t, s) V(\tau, t), \quad 0 \leq s<t \leq \tau \leq \mathrm{T},
$$

and, given any $g \in E_{1-\beta}^{\sharp}$, that $V(t, s) g \rightarrow g$ in $E_{1-\beta}^{\sharp}$ as $t-s \rightarrow 0+$. Indeed, this follows by interpolation, thanks to (1.4), from the strong continuity of $V$ on $E_{-\alpha}^{\sharp}$ and $E_{1-\alpha}^{\sharp}$ (cf. [3, Theorems II.4.4.1 and V.2.5.3]). Consequently, if $\left(y_{k}\right)$ is a converging sequence in $E_{1-\beta}^{\sharp}$, then

$$
\left(V\left(t_{k}, s_{k}\right)-1\right) y_{k} \rightarrow 0 \text { in } E_{1-\beta}^{\sharp} \quad \text { as } t_{k}-s_{k} \rightarrow 0+.
$$

Now let $M$ be a bounded subset of $L_{p^{\prime}}\left(J_{\mathrm{T}}, E_{-\alpha}^{\sharp}\right)$. Then (16.1) (with $\beta_{1}$ and $\varepsilon_{1}$ ) implies the boundedness of $V \circledast M$ in $C\left(J_{\mathrm{T}}, E_{1-\beta_{1}}^{\sharp}\right)$. By [3, Theorem V.1.5.1] the embedding $E_{1-\beta_{1}}^{\sharp} \hookrightarrow E_{1-\beta}^{\sharp}$ is compact. Hence, by the Arzelà-Ascoli theorem, it suffices to show that the set $V \circledast M$ is equicontinuous in $C\left(J_{T}, E_{1-\beta}^{\sharp}\right)$.

Assume to the contrary that there exist $0 \leq s_{k}<t_{k} \leq \mathrm{T}$ and $g_{k} \in M$ such that $t_{k}-s_{k} \rightarrow 0$ and

$$
\left\|V \circledast g_{k}\left(s_{k}\right)-V \circledast g_{k}\left(t_{k}\right)\right\|_{E_{1-\beta}^{\sharp}} \geq c>0, \quad k \in \mathbb{N} .
$$

Since the sequence $\left(V \circledast g_{k}\left(t_{k}\right)\right)$ is bounded in $E_{1-\beta_{1}}^{\sharp}$ we can assume that it is convergent in $E_{1-\beta}^{\sharp}$. Consequently,

$$
\begin{aligned}
\| V \circledast & g_{k}\left(s_{k}\right)-V \circledast g_{k}\left(t_{k}\right) \|_{E_{1-\beta}^{\sharp}} \\
& \leq\left\|\int_{s_{k}}^{t_{k}} V\left(\tau, s_{k}\right) g_{k}(\tau) d \tau\right\|_{E_{1-\beta}^{\sharp}}+\left\|\left(V\left(t_{k}, s_{k}\right)-1\right) V \circledast g_{k}\left(t_{k}\right)\right\|_{E_{1-\beta}^{\sharp}} \\
& \leq c\left(t_{k}-s_{k}\right)^{\varepsilon_{1}}+o(1)
\end{aligned}
$$

as $k \rightarrow \infty$, thanks to (17.1) (17.3). But this contradicts (17.4) and proves the assertion.

Suppose that $1 \leq r<\infty$ and $\alpha \leq \gamma<\beta$. Given $(x, f) \in E_{\beta-1 / r} \times L_{r}\left(J_{\mathrm{T}}, E_{\beta-1}\right)$, set $u(x, f):=u\left(f+x \otimes \delta_{0}\right)$. Then it is not difficult to verify that the proof of 5 . Theorem 5] carries over to the present situation. Hence

$$
((x, f) \mapsto u(x, f)) \in \mathcal{L}\left(E_{\beta-1 / r} \times L_{r}\left(J_{\mathrm{T}}, E_{\beta-1}\right), \mathbb{W}_{r}^{1}\left(J_{\mathrm{T}},\left(E_{\gamma-1}, E_{\gamma}\right)\right)\right) .
$$

Denoting by $\gamma_{0}$ the trace operator at $t=0$, we also write $\left(\partial+A, \gamma_{0}\right)^{-1}$ for the map (17.5), since $u(x, f)$ solves the nonautonomous initial value problem

$$
\dot{u}+A(t) u=f(t), \quad 0<t \leq \mathrm{T}, \quad u(u)=x .
$$

Now we can prove the analogue of Proposition 17.1 for strong solutions of (17.6).

Proposition 17.2. Suppose that $A_{0}$ has a compact resolvent.

(i) If $1<r<1 /(\beta-\alpha)$ and $1 \leq p<1 /(\alpha-\beta+1 / r)$, then

$$
\left(\partial+A, \gamma_{0}\right)^{-1} \in \mathcal{K}\left(E_{\beta-1 / r} \times L_{r}\left((0, \mathrm{~T}), E_{\beta-1}\right), L_{p}\left((0, \mathrm{~T}), E_{\alpha}\right)\right) .
$$


(ii) If $1 /(\beta-\alpha)<r<\infty$ and $0 \leq \rho<\beta-\alpha-1 / r$, then

$$
\left(\partial+A, \gamma_{0}\right)^{-1} \in \mathcal{K}\left(E_{\beta-1 / r} \times L_{r}\left((0, \mathrm{~T}), E_{\beta-1}\right), C^{\rho}\left([0, \mathrm{~T}], E_{\alpha}\right)\right) .
$$

Proof. In case (i) fix $\gamma \in(\alpha, \beta)$ such that $p<1 /(\alpha-\gamma+1 / r)$. In case (ii) choose $\gamma \in(\alpha, \beta)$ such that $1 /(\gamma-\alpha)<r$ and $\rho<\gamma-\alpha-1 / r$. Then the assertions follow from (17.5) and [5, Theorem 3].

\section{A perturbation theorem}

In this section we prove a general perturbation theorem for generators of analytic semigroups. It is the basis for the proof of the positivity result of the next section.

Theorem 18.1. Let $X_{0}$ and $X_{1}$ be Banach spaces such that $X_{1} \stackrel{d}{\hookrightarrow} X_{0}$, and suppose that $X_{\theta}=\left\{X_{0}, X_{1}\right\}_{\theta}$ for some $\theta \in(0,1)$ and some interpolation functor $\{\cdot, \cdot\}_{\theta}$ of exponent $\theta$. If $\mathrm{A} \in \mathcal{H}\left(X_{1}, X_{0}\right)$ and $\mathrm{B} \in \mathcal{L}\left(X_{1}, X_{\theta}\right)$, then $\mathrm{A}+\mathrm{B} \in \mathcal{H}\left(X_{1}, X_{0}\right)$. Furthermore, given $r>0$, there exists $\omega>0$ such that $\{z \in \mathbb{C} ; \operatorname{Re} z \geq \omega\}$ belongs to the resolvent set of $-(\mathrm{A}+\mathrm{B})$ for $\mathrm{B} \in \mathbb{B}_{r}:=r \mathbb{B}_{\mathcal{L}\left(X_{1}, X_{\theta}\right)}$, and the map

$$
\mathbb{B}_{r} \rightarrow \mathcal{L}\left(X_{0}, X_{1}\right), \quad \mathrm{B} \mapsto(\lambda+\mathrm{A}+\mathrm{B})^{-1},
$$

is analytic for each $\lambda$ with $\operatorname{Re} \lambda \geq \omega$.

Proof. Since $\mathrm{A} \in \mathcal{H}\left(E_{1}, E_{0}\right)$, there exist $\omega_{0}>0$ and $M \geq 1$ such that the half-plane $\left\{z \in \mathbb{C} ; \operatorname{Re} z \geq \omega_{0}\right\}$ belongs to the resolvent set of $-\mathrm{A}$, and

$$
R(\lambda):=(\lambda+\mathrm{A})^{-1} \in \mathcal{L}\left(X_{0}, X_{1}\right)
$$

with

$$
\|R(\lambda)\|_{\mathcal{L}\left(X_{j}, X_{k}\right)} \leq M|\lambda|^{k-j-1}, \quad \operatorname{Re} \lambda \geq \omega_{0}, \quad j, k \in\{0,1\}, \quad j \leq k
$$

(cf. [3. Section I.1.2]). Thus, by interpolation,

$$
\|R(\lambda)\|_{\mathcal{L}\left(X_{\theta}, X_{1}\right)} \leq \kappa M|\lambda|^{\theta-1}, \quad \operatorname{Re} \lambda \geq \omega_{0},
$$

for some $\kappa>0$. Consequently, for $k \geq 2$,

$$
\begin{aligned}
\left\|(\mathrm{B} R(\lambda))^{k}\right\|_{\mathcal{L}\left(X_{0}\right)} & \leq c\left\|(\mathrm{~B} R(\lambda))^{k}\right\|_{\mathcal{L}\left(X_{0}, X_{\theta}\right)} \\
& \leq c\|\mathrm{~B} R(\lambda)\|_{\mathcal{L}\left(X_{\theta}\right)}^{k-1}\|\mathrm{~B} R(\lambda)\|_{\mathcal{L}\left(X_{0}, X_{\theta}\right)} \\
& \leq c\left(r\|R(\lambda)\|_{\mathcal{L}\left(X_{\theta}, X_{1}\right)}\right)^{k-1} r\|R(\lambda)\|_{\mathcal{L}\left(X_{0}, X_{1}\right)} \\
& \leq c r M\left(r \kappa M|\lambda|^{\theta-1}\right)^{k-1}
\end{aligned}
$$

for $\operatorname{Re} \lambda \geq \omega_{0}$ and $\mathrm{B} \in \mathbb{B}_{r}$. Thus there exists $\omega:=\omega(r) \geq \omega_{0}$ with $r \kappa M|\lambda|^{\theta-1} \leq 1 / 2$ for $\operatorname{Re} \lambda \geq \omega$. Hence

$$
D(\lambda):=\sum_{k=2}^{\infty}(-1)^{k}(\mathrm{~B} R(\lambda))^{k} \in \mathcal{L}\left(X_{0}\right)
$$

and

$$
\|D(\lambda)\|_{\mathcal{L}\left(X_{0}\right)} \leq c r M, \quad \operatorname{Re} \lambda \geq \omega
$$

It is easily verified that

$$
(1+\mathrm{B} R(\lambda))(1-\mathrm{B} R(\lambda)+D(\lambda))=1=(1-\mathrm{B} R(\lambda)+D(\lambda))(1+\mathrm{B} R(\lambda))
$$

for $\operatorname{Re} \lambda \geq \omega$. Thus $1+\mathrm{B} R(\lambda)$ is invertible and

$$
(1+\mathrm{B} R(\lambda))^{-1}=1-\mathrm{B} R(\lambda)+D(\lambda) \in \mathcal{L}\left(X_{0}\right)
$$


with

$$
\left\|(1+\mathrm{B} R(\lambda))^{-1}\right\|_{\mathcal{L}\left(X_{0}\right)} \leq 1+2 \operatorname{cr} M=: M_{1}(r)
$$

for $\operatorname{Re} \lambda \geq \omega$ and $\mathrm{B} \in \mathbb{B}_{r}$. Now $\lambda+\mathrm{A}+\mathrm{B}=(1+\mathrm{B} R(\lambda))(\lambda+\mathrm{A})$ implies

$$
(\lambda+\mathrm{A}+\mathrm{B})^{-1}=R(\lambda)(1+\mathrm{B} R(\lambda))^{-1}, \quad \operatorname{Re} \lambda \geq \omega,
$$

and we infer from (18.2) and (18.3) that

$$
\left\|(\lambda+\mathrm{A}+\mathrm{B})^{-1}\right\|_{\mathcal{L}\left(X_{0}, X_{k}\right)} \leq M M_{1}(r)|\lambda|^{k-1}, \quad \operatorname{Re} \lambda \geq \omega, \quad \mathrm{B} \in \mathbb{B}_{r} .
$$

Thanks to [3, Remark I.1.2.1(a)], this proves that $\mathrm{A}+\mathrm{B} \in \mathcal{H}\left(X_{1}, X_{0}\right)$. Furthermore, given $\lambda$ with $\operatorname{Re} \lambda \geq \omega$, the analyticity of the map

$$
\mathcal{L}\left(X_{1}, X_{\theta}\right) \rightarrow \mathcal{L}\left(X_{0}\right), \quad \mathrm{B} \mapsto 1+\mathrm{B} R(\lambda),
$$

the analyticity of the inversion map $C \mapsto C^{-1}$, (18.3), and (18.4) imply the analyticity of (18.1).

\section{Positivity}

In this last section we presuppose hypotheses $(\mathrm{P} 0)-(\mathrm{P} 2)$ and use the notations of Part 2. Furthermore, given a Banach space $X_{0}$, we denote by $\mathcal{H}\left(X_{0}\right)$ the set of negative generators of strongly continuous analytic semigroups on $X_{0}$. Consequently, $\mathcal{H}\left(X_{1}, X_{0}\right)=\mathcal{H}\left(X_{0}\right) \cap \mathcal{L}\left(X_{1}, X_{0}\right)$ if $X_{1} \stackrel{d}{\hookrightarrow} X_{0}$. If $X_{0}$ is an ordered Banach space, then $\mathcal{H}^{+}\left(X_{0}\right)$ is the set of all resolvent positive operators in $\mathcal{H}\left(X_{0}\right)$, and $\mathcal{H}^{+}\left(X_{1}, X_{0}\right):=\mathcal{H}\left(X_{1}, X_{0}\right) \cap \mathcal{H}^{+}\left(X_{0}\right)$.

\section{Lower order perturbations of elliptic generators.}

Lemma 19.1. Suppose that

$$
\left(\vec{a}, \vec{c}, a_{0}, b\right) \in\left(L_{\infty}\right)^{n} \times\left(C_{0}^{1}\right)^{n} \times L_{\infty} \times C^{1}\left(\Gamma_{1}\right)
$$

and define $\left(\mathcal{A}^{*}, \mathcal{B}^{*}\right)$ by

$$
\mathcal{A}^{*} u:=\mathcal{A} u+\vec{a} \cdot \nabla u-\nabla \cdot(\vec{c} u)+a_{0} u, \quad \mathcal{B}^{*} u:=\mathcal{B} u+b \gamma u,
$$

where $b \mid \Gamma_{0}:=0$. Let $A_{0}^{*}$ be the $E_{0}$-realization of $\left(\mathcal{A}^{*}, \mathcal{B}^{*}\right)$. Then $A_{0}^{*} \in \mathcal{H}^{+}\left(E_{0}\right)$.

Proof. It is known that $A^{*} \in \mathcal{H}\left(E_{0}\right)$, and $\left(\lambda+A^{*}\right)^{-1} \in \mathcal{L}\left(L_{p}, W_{p}^{2}\right)$ for $p \in(1, \infty)$ and every sufficiently large $\lambda>0$. Fix any $\lambda \geq\left|a_{0}\right|_{\infty}+|\nabla \cdot \vec{c}|_{\infty}$ belonging to the resolvent set of $-A^{*}$. Then, given $f \in \mathcal{D}^{+}:=\{v \in \mathcal{D} ; v \geq 0\}$, it follows that $u:=\left(\lambda+A^{*}\right)^{-1} f \in W_{p}^{2}$ for $p>n$. Thus we infer from [1, Theorem 6.1], by observing that the proof given there remains valid under conditions (19.1), that $u \geq 0$, provided $\Omega$ is bounded. If $\Omega$ is unbounded, then we obtain $u \geq 0$ by using, in addition, the arguments of the proof of Lemma 3.1(iii). Since $\mathcal{D}^{+}$is dense in the positive cone of $E_{0}$, the assertion follows.

For $B \in \mathcal{L}\left(E_{\alpha}, E_{\beta-1}\right)$, we denote by $B_{0}$ the restriction of $B$ onto $E_{0}$, considered as a linear operator in $E_{0}$; that is, $B_{0}$ is the $E_{0}$-realization of $B$. We write

$$
\left[\left(E_{\xi}\left(A_{0}+B_{0}\right),\left(A_{0}+B_{0}\right)_{\xi}\right) ;-1 \leq \xi \leq 1\right]
$$

for the interpolation extrapolation scale generated by $\left(E_{0}, A_{0}+B_{0}\right)$ and the interpolation functors (3.3), if $A_{0}+B_{0} \in \mathcal{H}\left(E_{0}\right)$. We also set

$$
\begin{aligned}
\mathcal{H}_{\alpha, \beta}:=\left\{B \in \mathcal{L}\left(E_{\alpha}, E_{\beta-1}\right) ;\right. & A_{0}+B_{0} \in \mathcal{H}^{+}\left(E_{0}\right), \\
& \left.E_{\xi}\left(A_{0}+B_{0}\right) \doteq E_{\xi} \text { for } \xi \in\{\alpha, \beta-1\}\right\},
\end{aligned}
$$


and $\overline{\mathcal{H}}_{\alpha, \beta}$ is the closure of $\mathcal{H}_{\alpha, \beta}$ in $\mathcal{L}\left(E_{\alpha}, E_{\beta-1}\right)$. Observe that Theorem 18.1 implies $A+B \in \mathcal{H}\left(E_{\alpha}, E_{\alpha-1}\right)$ for $B \in \mathcal{L}\left(E_{\alpha}, E_{\beta-1}\right)$, hence for $B \in \overline{\mathcal{H}}_{\alpha, \beta}$.

Now we suppose that

- $s \in\left[0,2-n / q^{\prime}\right) \cap I_{q}, \nu_{0}, \nu_{1}, \rho \in[1, \infty)$;

- $\frac{1}{q^{\prime}} \leq \frac{1}{\nu_{0}} \leq \frac{1}{q^{\prime}}+\frac{s}{n}$;

- $\frac{1}{q^{\prime}} \leq \frac{1}{\nu_{1}} \leq \frac{1}{q^{\prime}}+\frac{s-1}{n}$ if $s \geq 1$;

- $0 \leq \frac{1}{\rho} \leq 1-\frac{1}{n-1}\left(\frac{n}{q}-s\right)$ if $s>1 / q$,

where the lower bounds for $1 / \nu_{0}$ and $1 / \nu_{1}$ are to be replaced by 0 if $\Omega$ is bounded. For abbreviation, we set $L_{(\nu, \infty)}:=L_{\nu}+L_{\infty}$ for $\nu \in[1, \infty)$. Then

$$
\mathfrak{B}:=\left\{\begin{aligned}
\{0\} \times\left(L_{\nu_{0}}\right)^{n} & \times L_{\left(\nu_{0}, \infty\right)} \times\{0\}, & 0 \leq s<1-n / q^{\prime}, \\
\{0\} \times\{0\} & \times L_{\left(\nu_{0}, \infty\right)} \times\{0\}, & 1-n / q^{\prime} \leq s<1 / q, \\
\{0\} \times\{0\} & \times L_{\left(\nu_{0}, \infty\right)} \times L_{\rho}\left(\Gamma_{1}\right), & 1 / q<s<1, \\
\left(L_{\left(\nu_{1}, \infty\right)}\right)^{n} \times\{0\} & \times L_{\left(\nu_{0}, \infty\right)} \times L_{\rho}\left(\Gamma_{1}\right), & 1 \leq s<2-n / q^{\prime} .
\end{aligned}\right.
$$

Given $\mathfrak{b}:=\left(\vec{a}, \vec{c}, a_{0}, b\right) \in \mathfrak{B}$, we define $B:=B(\mathfrak{b})$ by

$$
\langle v, B w\rangle:=\left\langle v, \vec{a} \cdot \nabla w+a_{0} w\right\rangle+\langle\nabla v, \vec{c} w\rangle+\langle\gamma v, b \gamma w\rangle_{\Gamma_{1}}
$$

for $(v, w) \in \mathcal{D}_{\mathcal{B}} \times W_{q, \mathcal{B}}^{s}$.

Recall that $2 \alpha=s$ and $2 \beta=\sigma$.

Lemma 19.2. Suppose that

$$
s<\sigma< \begin{cases}1-n / q^{\prime} & \text { if } s<1-n / q^{\prime}, \\ 2-n / q^{\prime} & \text { otherwise. }\end{cases}
$$

Then $B(\mathfrak{b}) \in \mathcal{L}\left(E_{\alpha}, E_{\beta-1}\right)$ for $\mathfrak{b} \in \mathfrak{B}$ and $(\mathfrak{b} \mapsto B(\mathfrak{b})) \in \mathcal{L}\left(\mathfrak{B}, \mathcal{L}\left(E_{\alpha}, E_{\beta-1}\right)\right)$.

Proof. It is an easy consequence of (19.3), Lemma 4.1, and Hölder's inequality that

$$
W_{q^{\prime}, \mathcal{B}}^{2-\sigma} \times W_{q, \mathcal{B}}^{s} \rightarrow \mathbb{R}, \quad(v, w) \mapsto\langle v, B(\mathfrak{b}) w\rangle
$$

is a well-defined continuous bilinear form depending continuously on $\mathfrak{b} \in \mathfrak{B}$. Since $W_{q^{\prime}, \mathcal{B}}^{2-\sigma} \times W_{q, \mathcal{B}}^{s}=E_{1-\beta}^{\sharp} \times E_{\alpha}$, the assertion follows.

Lemma 19.3. Let assumptions (19.3) and (19.4) be satisfied. Then $B(\mathfrak{b}) \in \overline{\mathcal{H}}_{\alpha, \beta}$ for $\mathfrak{b} \in \mathfrak{B}$.

Proof. Let $\mathfrak{b} \in \mathfrak{B}$ be given. Since $C_{0}^{1} \times C^{1}\left(\Gamma_{1}\right)$ is dense in $L_{\nu_{0}} \times L_{\rho}\left(\Gamma_{1}\right)$, there exist

$$
\mathfrak{b}_{j} \in\left(L_{\infty}\right)^{n} \times\left(C_{0}^{1}\right)^{n} \times L_{\infty} \times C^{1}\left(\Gamma_{1}\right), \quad j \in \mathbb{N},
$$

such that $\mathfrak{b}_{j} \rightarrow \mathfrak{b}$ in $\mathfrak{B}$. Define $\left(\mathcal{A}_{j}^{*}, \mathcal{B}_{j}^{*}\right)$ as in Lemma 19.1, but with $\left(\vec{a}, \vec{c}, a_{0}, b\right)$ replaced by $\mathfrak{b}_{j}$. Then that lemma guarantees that $A_{0, j}^{*}$, the $E_{0}$-realization of $\left(\mathcal{A}_{j}^{*}, \mathcal{B}_{j}^{*}\right)$, belongs to $\mathcal{H}^{+}\left(E_{0}\right)$. Observe that, because $\vec{c}_{j} \mid \Gamma=0$, the formally dual problem $\left(\left(\mathcal{A}_{j}^{*}\right)^{\sharp},\left(\mathcal{B}_{j}^{*}\right)^{\sharp}\right)$ satisfies $\left(\mathcal{B}_{j}^{*}\right)^{\sharp}=\mathcal{B}_{j}^{*}$ (cf. [2, Section 7]). Since $\mathcal{B}_{j}^{*}=\mathcal{B}$ if $b=b_{j}=0$, it follows from (3.2) that

$$
E_{\xi}\left(A_{0, j}^{*}\right)=E_{\xi}, \quad \xi \in\{\alpha, \beta-1\}, \quad j \in \mathbb{N},
$$


provided we choose $\mathfrak{b}_{j}$ such that $b_{j}=0$ if $b=0$, which we do. (Note that $E_{1}\left(A_{0, j}^{*}\right)$ is different from $E_{1}$, in general.)

Lemma 19.2 implies $B_{j}:=B\left(\mathfrak{b}_{j}\right) \in \mathcal{L}\left(E_{\alpha}, E_{\beta-1}\right)$. Furthermore, it follows from (19.5) that $A_{\alpha, j}^{*}$, the $E_{\alpha}$-realization of $A_{0, j}^{*}$, is given by $A+B_{j}$ (cf. [2, Theorem 8.3]). This proves that $B_{j} \in \mathcal{H}_{\alpha, \beta}$. Now the assertion follows from Lemma 19.2.

The generalized maximum principle. Now we fix any $T>0$, set $J:=[0, T]$, and introduce the following assumption:

- $1 \leq r<\infty$ with $\sigma-2 / r \in I_{q}$;

- $\frac{1}{\pi}:= \begin{cases}1 / r-1 / p & \text { if } r \leq p<2 /(s-\sigma+2 / r), \\ 1 / r & \text { if } r>2 /(\sigma-s)\end{cases}$

- $0 \leq 2 p<\sigma-s-2 / r$ if $r>2 /(\sigma-s)$;

- $\mathfrak{b}=\left(\vec{a}, \vec{c}, a_{0}, b\right) \in L_{\pi}(J, \mathfrak{B})$.

We also set

$$
M_{q}:=\left\{\begin{array}{lr}
\overrightarrow{\mathcal{M}}_{\text {sing }}, & 0 \leq s<1-n / q^{\prime}, \\
\left(\mathcal{M}+L_{q}\right) \times\{0\} \times \mathcal{M}\left(\Gamma_{1}\right), & 1-n / q^{\prime} \leq s<2-n / q^{\prime} .
\end{array}\right.
$$

Finally, we put

$$
M_{r, q, \sigma}:=M_{r, q, \sigma}(J):=\left\{\begin{aligned}
\{0\} \times \mathcal{M}\left(J, M_{q}\right), & r=1, \\
W_{q, \mathcal{B}}^{\sigma-2 / r} \times L_{r}\left(J, M_{q}\right), & r>1 .
\end{aligned}\right.
$$

Then, given $\left(u^{0}, \vec{\mu}\right) \in M_{r, q, \sigma}$, we consider the nonautonomous linear problem

$$
\begin{aligned}
& \partial_{t} u+\mathcal{A}^{*}(t) u=\mu_{\Omega}+u^{0} \otimes \delta_{0} \quad \text { in } \quad \Omega \times J, \\
& \mathcal{B}^{*}(t) u=\mu_{\Gamma} \quad \text { on } \quad \Gamma \times J,
\end{aligned}
$$

where $\left(\mathcal{A}^{*}(t), \mathcal{B}^{*}(t)\right)$ is defined by 19.2).

After these preparations we can prove the main result of this section, the following generalized maximum principle. Although the above hypotheses seem to be rather technical, they are precisely the ones one arrives at by linearizing problems (4.23) and (4.26).

Theorem 19.4. Let assumptions (19.6) be satisfied and suppose that $\left(u^{0}, \vec{\mu}\right)$ belongs to $M_{r, q, \sigma}$. Then problem (19.7) has a unique $X_{p, q}^{s}$-solution on J. It is positive if $\left(u^{0}, \vec{\mu}\right) \geq 0$.

Proof. Given $\mathfrak{b} \in L_{\pi}(J, \mathfrak{B})$, define $B(\mathfrak{b})$ pointwise, that is, $B(\mathfrak{b})(t):=B(\mathfrak{b}(t))$ for $t \in J$. Lemma 19.2 easily implies that $B(\mathfrak{b}) \in L_{\pi}\left(J, \mathcal{L}\left(E_{\alpha}, E_{\beta-1}\right)\right)$ and

$$
(\mathfrak{b} \mapsto B(\mathfrak{b})) \in \mathcal{L}\left(L_{\pi}(J, \mathfrak{B}), L_{\pi}\left(J, \mathcal{L}\left(E_{\alpha}, E_{\beta-1}\right)\right)\right) .
$$

Set

where

$$
\mu(u):=-B(\mathfrak{b}) u+\widetilde{\chi}(\vec{\mu})+u^{0} \otimes \delta_{0}, \quad u \in X_{p, q}^{s}(J),
$$

$$
\widetilde{\chi}(\vec{\mu}):=\left\{\begin{aligned}
\chi_{\operatorname{sing}}(0, \vec{\mu}) & \text { if } s<1-n / q^{\prime}, \\
\chi(0, \vec{\mu}) & \text { otherwise. }
\end{aligned}\right.
$$

Then it follows from (19.8), Lemma 15.1(iii), the assumptions on $\left(u^{0}, \vec{\mu}\right)$, (4.15), (4.22), and Remark 4.3)(a) that

$$
\mu \in C_{b}^{1-}\left(X_{p, q}^{s}(J), \mathcal{M}\left(J, E_{\alpha-1}\right)\right) \quad \text { if } r=1,
$$


and

$$
\mu \in C_{b}^{1-}\left(X_{p, q}^{s}(J), L_{r}\left(J, E_{\alpha-1}\right)\right) \quad \text { otherwise. }
$$

Furthermore, Theorems 4.4 and 4.5 guarantee that $u \in X_{p, q}^{s}(J)$ is an $X_{p, q}^{s}$-solution of (19.7) on $J$ iff it is such a solution of $\dot{u}+A u=\mu(u)$ on $J$. Hence the asserted unique global solvability of (19.7) is a consequence of Theorems 3.23 .4 and Re$\operatorname{mark} 2.4(\mathrm{~b})$.

Let $\mathfrak{b} \in L_{\pi}(J, \mathfrak{B})$ be fixed. Then there exists a sequence $\left(\mathfrak{b}_{j}\right)$ in $C^{1}(J, \mathfrak{B})$ converging in $L_{\pi}(J, \mathfrak{B})$ towards $\mathfrak{b}$. Set $B_{j}:=B\left(\mathfrak{b}_{j}\right)$ for $j \in \mathbb{N}$, and $B:=B(\mathfrak{b})$. Then we infer from (19.8) that $B_{j} \rightarrow B$ in $L_{\pi}\left(J, \mathcal{L}\left(E_{\alpha}, E_{\beta-1}\right)\right)$. Also define $\mu_{j}$ by replacing $B$ in the definition of $\mu$ by $B_{j}$. It is an obvious consequence of Lemma 15.1(iii) that $\mu_{j} \rightarrow \mu$ in $C_{b}^{1-}\left(X_{p, q}^{s}(J), \mathcal{M}\left(J, E_{\alpha-1}\right)\right)$, resp. in $C_{b}^{1-}\left(X_{p, q}^{s}(J), L_{r}\left(J, E_{\alpha-1}\right)\right)$, uniformly on bounded subsets of $X_{p, q}^{s}$. Hence the continuity assertions of Theorems 3.2 3.4 imply that $u_{j} \rightarrow u$ in $X_{p, q}^{s}(J)$, where $u_{j}$ is the $X_{p, q}^{s}$-solution of (19.7) with $\mathfrak{b}$ replaced by $\mathfrak{b}_{j}$. Consequently, $u_{j} \geq 0$ for all $j \in \mathbb{N}$ implies $u \geq 0$. Hence it suffices to prove the positivity assertion for the case that $\mathfrak{b}=\left(\vec{a}, \vec{c}, a_{0}, b\right) \in C^{1}(J, \mathfrak{B})$. It is an obvious consequence of Lemma 19.2 that this implies

$$
B \in C^{1}\left(J, \mathcal{L}\left(E_{\alpha}, E_{\beta-1}\right)\right) .
$$

Thus let (19.9) be satisfied. Then we infer from Theorem 18.1 that

$$
(t \mapsto A(t):=A+B(t)) \in C^{1}\left(J, \mathcal{H}\left(E_{\alpha}, E_{\alpha-1}\right)\right) .
$$

Furthermore, Lemma 19.3 shows that $A(t)$ is resolvent positive for each $t \in J$. Now we deduce from Proposition 16.3 that the nonautonomous problem

$$
\dot{v}+A(t) v=\tilde{\chi}(\vec{\mu})+u^{0} \otimes \delta_{0}, \quad t \in J
$$

has a unique weak $L_{1}\left(E_{\alpha}\right)$-solution $\bar{u}$, and that it is positive if $\widetilde{\chi}(\vec{\mu})+u^{0} \otimes \delta_{0} \geq 0$. Note that, thanks to Remark 4.3 (b), the latter inequality is satisfied if $\left(u^{0}, \vec{\mu}\right) \geq 0$. Thus $\bar{u} \geq 0$ if $\left(u^{0}, \vec{\mu}\right) \geq 0$. Clearly, (19.10) coincides with the weak formulation of (19.7). Thus the unique $X_{p, q}^{s}$-solution $u$ of (19.7) on $J$ is a weak $L_{1}\left(E_{\alpha}\right)$ solution of (19.10). Consequently, $u=\bar{u}$ by uniqueness, which shows that $u \geq 0$ if $\left(u^{0}, \vec{\mu}\right) \geq 0$. This proves everything.

\section{REFERENCES}

1. H. Amann, Dual semigroups and second order linear elliptic boundary value problems, Israel J. Math. 45 (1983), 225-254. MR 85i:35043

2. N Nonhomogeneous linear and quasilinear elliptic and parabolic boundary value problems, Function Spaces, Differential Operators and Nonlinear Analysis (H.J. Schmeisser and H. Triebel, eds.), Teubner, Stuttgart, Leipzig, 1993, 9-126. MR 94m:35153

3. L Linear and quasilinear parabolic problems, volume I: Abstract linear theory, Birkhäuser, Basel, 1995. MR 96g:34088

4. _ On the strong solvability of the Navier-Stokes equations, J. Math. Fluid Mech. 2 (2000), 16-98. MR 2002b:76028

5 . L Linear parabolic problems involving measures, Rev. R. Acad. Cien. Serie A. Mat. 95 (2001), 85-119.

6. H. Amann and P. Quittner, Elliptic boundary value problems involving measures: existence, regularity, and multiplicity, Advances in Diff. Equations 3 (1998), 753-813. MR 2000a:35063

7. J.M. Arrieta, A.N. Carvalho and A. Rodríguez-Bernal, Parabolic problems with nonlinear boundary conditions and critical nonlinearities, J. Differ. Equations 156 (1999), 376-406. MR 2000f:35075

8. J.M. Arrieta, P. Quittner and A. Rodríguez-Bernal, Parabolic problems with dynamical boundary condition and singular initial data, Differ. Integral Equations 14 (2001), 1487-1510. MR 2002h:35149 
9. J. Baek and M. Kwak, Singular solutions of semilinear parabolic equations in several space dimensions, J. Korean Math. Soc. 34 (1997), 1049-1064. MR 98i:35083

10. P. Baras and M. Pierre, Critère d'existence de solutions positives pour des équations semilinéaires non monotones, Analyse Non Linéaire, Ann. Inst. H. Poincaré 2 (1985), 185-212. MR 87j:45032

11. M. Ben-Artzi, P. Souplet and F.B. Weissler, Sur la non-existence et la non-unicité des solutions du problème de Cauchy pour une équation parabolique semi-linéaire, C. R. Acad. Sci. Paris Sér. I Math. 329 (1999), 371-376. MR 2000i:35077

12. - The local theory for viscous Hamilton-Jacobi equations in Lebesgue spaces, J. Math. Pures et Appl. 81 (2002), 343-378.

13. H.A. Biagioni, L. Cadeddu and T. Gramchev, Parabolic equations with conservative nonlinear term and singular initial data, Nonlinear Anal. TMA 30 (1997), 2489-2496. MR 98k:35110

14. H.A. Biagioni and T. Gramchev, Evolution PDE with elliptic dissipative terms: critical index for singular initial data, self-similar solutions and analytic regularity, C. R. Acad. Sci. Paris Sér. I Math. 327 (1998), 41-46. MR 99k:35081.

15. L. Boccardo, A. Dall'Aglio, Th. Gallouet and L. Orsina, Existence and regularity results for some nonlinear parabolic equations, Adv. Math. Sci. Appl. 9 (1999), 1017-1031. MR 2000h:35085

16. H. Brezis and X. Cabré, Some simple nonlinear PDE's without solutions, Bolletino U.M.I. (8) 1-B (1998), 223-262. MR 99j:35001

17. H. Brezis and T. Cazenave, A nonlinear heat equation with singular initial data, J. Anal. Math. 68 (1996), 277-304. MR 97f:35092

18. H. Brezis and A. Friedman, Nonlinear parabolic equations involving measures as initial conditions, J. Math. Pures et Appl. 62 (1983), 73-97. MR 84g:35093

19. J. Droniou and J.-P. Raymond, Optimal pointwise control of semilinear parabolic equations, Nonlinear Analysis 39 (2000), 135-156. MR 2000i:49007

20. E. Hewitt and K. Stromberg, Real and abstract analysis, Springer, Berlin - Heidelberg - New York, 1965. MR 32:5826

21. O.A. Ladyženskaja, V.A. Solonnikov, and N.N. Ural'ceva, Linear and quasilinear equations of parabolic type, Amer. Math. Soc., Transl. Math. Monographs, Providence, R.I., 1968. MR 39:3159a

22. P. Quittner, Global existence for semilinear parabolic problems, Adv. Math. Sci. Appl. 10 (2000), 643-660. MR 2002a:35105

23. P. Quittner and $\mathrm{Ph}$. Souplet, Admissible $L_{p}$ norms for local existence and for continuation in semilinear parabolic systems are not the same, Proc. Royal Soc. Edinburgh A 131 (2001), 1435-1456. MR 2003a:35098

24. F. Ribaud, Cauchy problem for parabolic semilinear equations with initial data in $H_{p}^{s}\left(\mathbb{R}^{n}\right)$, C. R. Acad. Sci. Paris Sér. I Math. 322 (1996), 25-30. MR 98c:35079

25. - Semilinear parabolic equations with distributions as initial data, Discrete and Cont. Dyn. Systems 3 (1997), 305-316. MR 97m:35127

26. L. Véron, Singularities of Solutions of Second Order Quasilinear Equations, Pitman Research Notes in Math., \#353, Longman Sci. \& Tech., Harlow, 1996. MR 98b:35053

27. F. Weissler, Local existence and nonexistence for semilinear parabolic equations in $L^{p}$, Indiana Univ. Math. J. 29 (1980), 79-102. MR 81c:35072

28. Q.S. Zhang and Z. Zhao, Singular solutions of semilinear elliptic and parabolic equations, Math. Ann. 310 (1998), 777-794. MR 99e:35078

Institut FÜr Mathematik, Universität ZÜrich, WinterthuRERStr. 190, CH-8057 Zürich, SWITZERLAND

E-mail address: amann@math.unizh.ch

Institute of Applied Mathematics, Comenius University, SK-84248 Bratislava, SloVAKIA

E-mail address: quittner@fmph.uniba.sk 